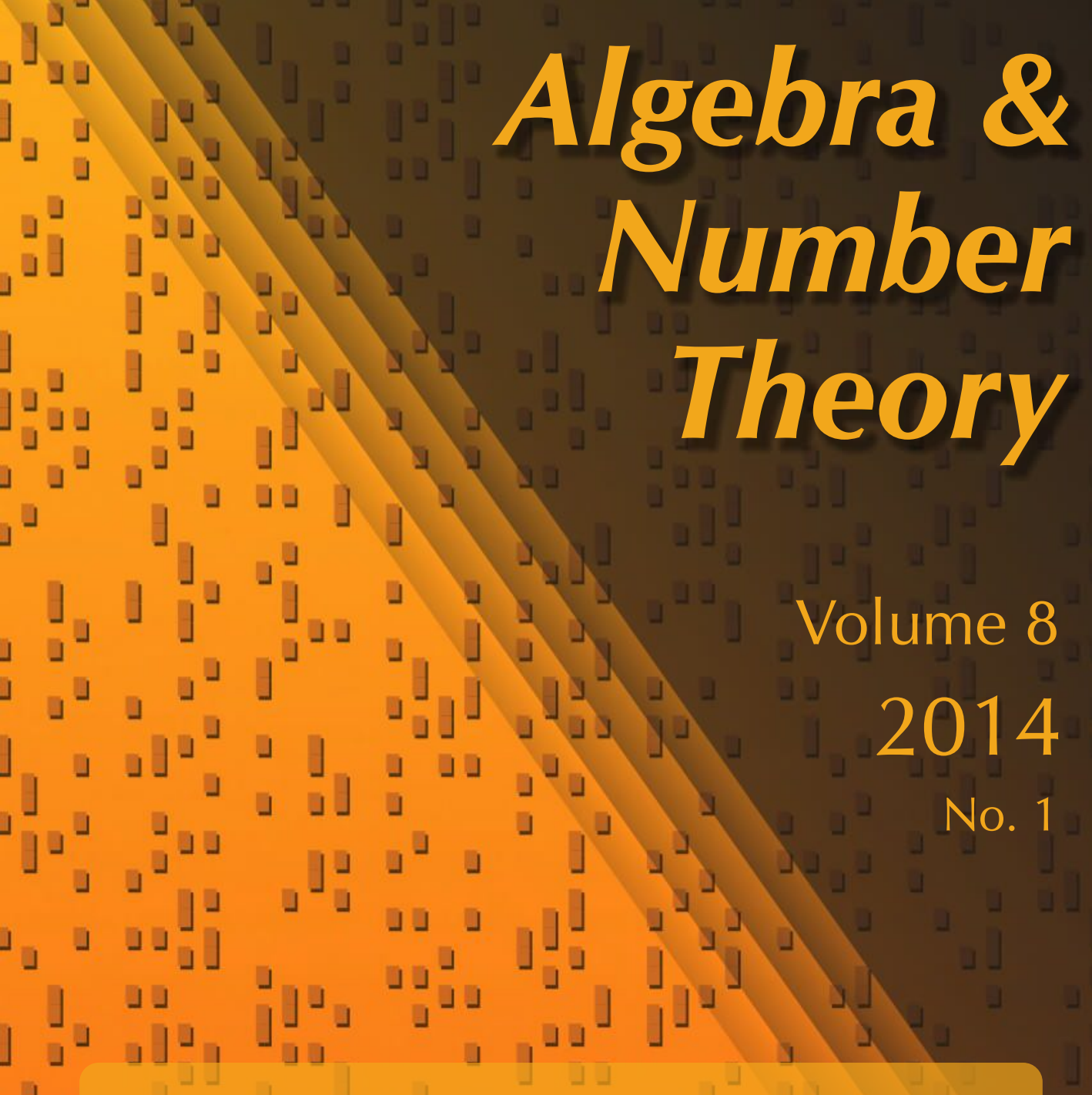

Polynomial bounds for Arakelov invariants of Belyi curves

Ariyan Javanpeykar

\lrcorner$\lrcorner$
Appendix by Peter Bruin

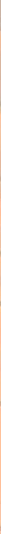




\title{
Polynomial bounds for Arakelov invariants of Belyi curves
}

\author{
Ariyan Javanpeykar \\ Appendix by Peter Bruin
}

\begin{abstract}
We explicitly bound the Faltings height of a curve over $\overline{\mathbb{Q}}$ polynomially in its Belyi degree. Similar bounds are proven for three other Arakelov invariants: the discriminant, Faltings' delta invariant and the self-intersection of the dualising sheaf. Our results allow us to explicitly bound these Arakelov invariants for modular curves, Hurwitz curves and Fermat curves in terms of their genus. Moreover, as an application, we show that the Couveignes-Edixhoven-Bruin algorithm to compute coefficients of modular forms for congruence subgroups of $\mathrm{SL}_{2}(\mathbb{Z})$ runs in polynomial time under the Riemann hypothesis for $\zeta$-functions of number fields. This was known before only for certain congruence subgroups. Finally, we use our results to prove a conjecture of Edixhoven, de Jong and Schepers on the Faltings height of a cover of $\mathbb{P}_{\mathbb{Z}}^{1}$ with fixed branch locus.
\end{abstract}

\section{Introduction and statement of results}

We prove that stable Arakelov invariants of a curve over a number field are polynomial in the Belyi degree. We apply our results to give algorithmic, geometric and Diophantine applications.

1.1. Bounds for Arakelov invariants of three-point covers. Let $\overline{\mathbb{Q}}$ be an algebraic closure of the field of rational numbers $\mathbb{Q}$. Let $X$ be a smooth projective connected curve over $\overline{\mathbb{Q}}$ of genus $g$. Belyi [1979] proved that there exists a finite morphism $X \rightarrow \mathbb{P}_{\bar{Q}}^{1}$ ramified over at most three points. Let $\operatorname{deg}_{B}(X)$ denote the Belyi degree of $X$, i.e., the minimal degree of a finite morphism $X \rightarrow \mathbb{P}_{\bar{Q}}^{1}$ unramified over $\mathbb{P}_{\overline{\mathbb{Q}}}^{1} \backslash\{0,1, \infty\}$. Since the topological fundamental group of the projective line $\mathbb{P}^{1}(\mathbb{C})$ minus three points is finitely generated, the set of $\overline{\mathbb{Q}}$-isomorphism classes of curves with bounded Belyi degree is finite.

MSC2010: primary 14G40; secondary 11G30, 11G32, 11G50, 14H55, 37P30.

Keywords: Arakelov theory, Arakelov-Green functions, Wronskian differential, Belyi degree, arithmetic surfaces, Riemann surfaces, curves, Arakelov invariants, Faltings height, discriminant, Faltings' delta invariant, self-intersection of the dualising sheaf, branched covers. 
We prove that, if $g \geq 1$, the Faltings height $h_{\mathrm{Fal}}(X)$, the Faltings delta invariant $\delta_{\mathrm{Fal}}(X)$, the discriminant $\Delta(X)$ and the self-intersection of the dualising sheaf $e(X)$ are bounded by a polynomial in $\operatorname{deg}_{B}(X)$; the precise definitions of these Arakelov invariants of $X$ are given in Section 2.3.

Theorem 1.1.1. For any smooth projective connected curve $X$ over $\overline{\mathbb{Q}}$ of genus $g \geq 1$,

$$
\begin{aligned}
-\log (2 \pi) g & \leq h_{\mathrm{Fal}}(X) \leq 13 \cdot 10^{6} g \operatorname{deg}_{B}(X)^{5}, \\
0 & \leq e(X) \leq 3 \cdot 10^{7}(g-1) \operatorname{deg}_{B}(X)^{5}, \\
0 & \leq \Delta(X) \leq 5 \cdot 10^{8} g^{2} \operatorname{deg}_{B}(X)^{5}, \\
-10^{8} g^{2} \operatorname{deg}_{B}(X)^{5} & \leq \delta_{\mathrm{Fal}}(X) \leq 2 \cdot 10^{8} g \operatorname{deg}_{B}(X)^{5} .
\end{aligned}
$$

The Arakelov invariants in Theorem 1.1.1 all have a different flavour to them. For example, the Faltings height $h_{\mathrm{Fal}}(X)$ plays a key role in Faltings' proof of his finiteness theorem on abelian varieties; see [Faltings 1983]. On the other hand, the strict positivity of $e(X)$ (when $g \geq 2$ ) is related to the Bogomolov conjecture; see [Szpiro $1990 \mathrm{~b}]$. The discriminant $\Delta(X)$ "measures" the bad reduction of the curve $X / \overline{\mathbb{Q}}$ and appears in the discriminant conjecture of Szpiro [1990a] for semistable elliptic curves. Finally, as was remarked by Faltings [1984, Introduction], Faltings' delta invariant $\delta_{\mathrm{Fal}}(X)$ can be viewed as the minus logarithm of a "distance" to the boundary of the moduli space of compact connected Riemann surfaces of genus $g$.

We were first led to investigate this problem by work of Edixhoven, de Jong and Schepers on covers of complex algebraic surfaces with fixed branch locus; see [Edixhoven et al. 2010]. They conjectured an arithmetic analogue [Edixhoven et al. 2010, Conjecture 5.1] of their main theorem (Theorem 1.1 in [loc. cit.]). We use our results to prove this conjecture; see Section 6 for a more precise statement.

1.2. Outline of proof. To prove Theorem 1.1.1, we will use Arakelov theory for curves over a number field $K$. To apply Arakelov theory in this context, we will work with arithmetic surfaces associated to such curves, i.e., regular projective models over the ring of integers $O_{K}$ of $K$. We refer the reader to Section 2.2 for precise definitions and basic properties of Arakelov's intersection pairing on an arithmetic surface. Then, for any smooth projective connected curve $X$ over $\overline{\mathbb{Q}}$ of genus $g \geq 1$, we define the Faltings height $h_{\mathrm{Fal}}(X)$, the discriminant $\Delta(X)$, Faltings' delta invariant $\delta_{\mathrm{Fal}}(X)$ and the self-intersection of the dualising sheaf $e(X)$ in Section 2.3. These are the four Arakelov invariants appearing in Theorem 1.1.1.

We introduce two functions on $X(\overline{\mathbb{Q}})$ in Section 2.3: the canonical Arakelov height function and the Arakelov norm of the Wronskian differential. We show that, to prove Theorem 1.1.1, it suffices to bound the canonical height of some non-Weierstrass point and the Arakelov norm of the Wronskian differential at this point; see Theorem 2.4.1 for a precise statement. 
We estimate Arakelov-Green functions and Arakelov norms of Wronskian differentials on finite étale covers of the modular curve $Y(2)$ in Theorem 3.4.5 and Proposition 3.5.1, respectively. In our proof, we use an explicit version of a result of Merkl on the Arakelov-Green function; see Theorem 3.1.2. This version of Merkl's theorem was obtained by Peter Bruin in his master's thesis. The proof of this version of Merkl's theorem is reproduced in the Appendix by Peter Bruin.

In Section 4, we prove the existence of a non-Weierstrass point on $X$ of bounded height; see Theorem 4.5.2. The proof of Theorem 4.5.2 relies on our bounds for Arakelov-Green functions (Theorem 3.4.5), the existence of a "wild" model (Theorem 4.3.2) and a generalisation of Dedekind's discriminant conjecture for discrete valuation rings of characteristic 0 (Proposition 4.1.1), which we attribute to Lenstra.

A precise combination of the above results constitutes the proof of Theorem 1.1.1 given in Section 4.6.

\subsection{Arakelov invariants of covers of curves with fixed branch locus. We apply}

Theorem 1.1.1 to prove explicit bounds for the height of a cover of curves. Let us be more precise.

For any finite subset $B \subset \mathbb{P}^{1}(\overline{\mathbb{Q}})$ and integer $d \geq 1$, the set of smooth projective connected curves $X$ over $\overline{\mathbb{Q}}$ such that there exists a finite morphism $X \rightarrow \mathbb{P}_{\overline{\mathbb{Q}}}^{1}$ étale over $\mathbb{P}_{\overline{\mathbb{Q}}}^{1}-B$ of degree $d$ is finite. In particular, the Faltings height of $X$ is bounded by a real number depending only on $B$ and $d$. In this section, we give an explicit version of this statement. To state our result, we need to define the height of $B$.

The (exponential) height $H(\alpha)$ of an element $\alpha$ in $\overline{\mathbb{Q}}$ is defined as $H(\alpha)=$ $\left(\prod_{v} \max \left(1,\|\alpha\|_{v}\right)\right)^{1 /[K: \mathbb{Q}]}$. Here $K$ is a number field containing $\alpha$ and the product runs over the set of normalised valuations $v$ of $K$. (As in [Khadjavi 2002, Section 2], we require our normalisation to be such that the product formula holds.) For any finite set $B \subset \mathbb{P}^{1}(\overline{\mathbb{Q}})$, define the height of $B$ as $H_{B}=\max \{H(\alpha): \alpha \in B\}$.

Theorem 1.3.1. Let $U$ be a nonempty open subscheme in $\mathbb{P}_{\overline{\mathbb{Q}}}^{1}$ with complement $B \subset \mathbb{P}^{1}(\overline{\mathbb{Q}})$. Let $N$ be the number of elements in the orbit of $B$ under the action of $\operatorname{Gal}(\overline{\mathbb{Q}} / \mathbb{Q})$. Then, for any finite morphism $\pi: Y \rightarrow \mathbb{P}_{\overline{\mathbb{Q}}}^{1}$ étale over $U$, where $Y$ is a smooth projective connected curve over $\overline{\mathbb{Q}}$ of genus $g \geq 1$,

$$
\begin{aligned}
&-\log (2 \pi) g \leq h_{\mathrm{Fal}}(Y) \leq 13 \cdot 10^{6} g\left(4 N H_{B}\right)^{45 N^{3} 2^{N-2} N !}(\operatorname{deg} \pi)^{5}, \\
& 0 \leq e(Y) \leq 3 \cdot 10^{7}(g-1)\left(4 N H_{B}\right)^{45 N^{3} 2^{N-2} N !}(\operatorname{deg} \pi)^{5}, \\
& 0 \leq \Delta(Y) \leq 5 \cdot 10^{8} g^{2}\left(4 N H_{B}\right)^{45 N^{3} 2^{N-2} N !}(\operatorname{deg} \pi)^{5}, \\
&-10^{8} g^{2}\left(4 N H_{B}\right)^{45 N^{3} 2^{N-2} N !}(\operatorname{deg} \pi)^{5} \\
& \\
& \leq \delta_{\mathrm{Fal}}(Y) \leq 2 \cdot 10^{8} g\left(4 N H_{B}\right)^{45 N^{3} 2^{N-2} N !}(\operatorname{deg} \pi)^{5} .
\end{aligned}
$$


Theorem 1.3.1 is a consequence of Theorem 6.0.4. Note that in Theorem 6.0.4 we consider branched covers of any curve over $\overline{\mathbb{Q}}$ (i.e., not only $\mathbb{P}_{\overline{\mathbb{Q}}}^{1}$ ). We use Theorem 1.3.1 to prove [Edixhoven et al. 2010, Conjecture 5.1].

1.4. Diophantine application. Explicit bounds for Arakelov invariants of curves of genus $g \geq 2$ over a number field $K$ and with bad reduction outside a finite set $S$ of finite places of $K$ imply famous conjectures in Diophantine geometry such as the effective Mordell conjecture and the effective Shafarevich conjecture; see [Rémond 1999] and [Szpiro 1985a]. We note that Theorem 1.1.1 shows that one "could" replace Arakelov invariants by the Belyi degree to prove these conjectures. We use this philosophy to deal with cyclic covers of prime degree. In fact, in [Javanpeykar and von Känel 2013], we utilise Theorem 1.1.1 and the theory of logarithmic forms to prove the small points conjecture of Szpiro [1985c, p. 284; 1986] for curves that are cyclic covers of the projective line of prime degree; see [Javanpeykar and von Känel 2013, Theorem 3.1] for a precise statement. In particular, we prove Szpiro's small points conjecture for hyperelliptic curves.

\subsection{Modular curves, Fermat curves, Hurwitz curves and Galois Belyi curves.}

Let $X$ be a smooth projective connected curve over $\overline{\mathbb{Q}}$ of genus $g \geq 2$. We say that $X$ is a Fermat curve if there exists an integer $n \geq 4$ such that $X$ is isomorphic to the planar curve $\left\{x^{n}+y^{n}=z^{n}\right\}$. Moreover, we say that $X$ is a Hurwitz curve if \# Aut $(X)=84(g-1)$. Also, we say that $X$ is a Galois Belyi curve if the quotient $X / \operatorname{Aut}(X)$ is isomorphic to $\mathbb{P}_{\overline{\mathbb{Q}}}^{1}$ and the morphism $X \rightarrow X / \operatorname{Aut}(X)$ is ramified over exactly three points; see [Clark and Voight 2011, Proposition 2.4] or [Wolfart 1997]. Note that Fermat curves and Hurwitz curves are Galois Belyi curves. Finally, we say that $X$ is a modular curve if $X_{\mathbb{C}}$ is a classical congruence modular curve with respect to some (hence any) embedding $\overline{\mathbb{Q}} \rightarrow \mathbb{C}$.

If $X$ is a Galois Belyi curve, we have $\operatorname{deg}_{B}(X) \leq 84(g-1)$. Zograf [1991] proved that, if $X$ is a modular curve, then $\operatorname{deg}_{B}(X) \leq 128(g+1)$. Combining these bounds with Theorem 1.1.1 we obtain the following corollary:

Corollary 1.5.1. Let $X$ be a smooth projective connected curve over $\overline{\mathbb{Q}}$ of genus $g \geq 1$. Suppose that $X$ is a modular curve or Galois Belyi curve. Then

$$
\max \left(h_{\mathrm{Fal}}(X), e(X), \Delta(X),\left|\delta_{\mathrm{Fal}}(X)\right|\right) \leq 2 \cdot 10^{19} g^{2}(g+1)^{5} .
$$

Remark 1.5.2. Let $\Gamma \subset \mathrm{SL}_{2}(\mathbb{Z})$ be a finite-index subgroup, and let $X$ be the compactification of $\Gamma \backslash \mathbb{W}$ obtained by adding the cusps, where $\Gamma$ acts on the complex upper half-plane $\mathbb{H}$ via Möbius transformations. Let $X(1)$ denote the compactification of $\mathrm{SL}_{2}(\mathbb{Z}) \backslash \mathbb{M}$. The inclusion $\Gamma \subset \mathrm{SL}_{2}(\mathbb{Z})$ induces a morphism $X \rightarrow X(1)$. For $\overline{\mathbb{Q}} \subset \mathbb{C}$ an embedding, there is a unique finite morphism $Y \rightarrow \mathbb{P}_{\overline{\mathbb{Q}}}^{1}$ of smooth projective connected curves over $\overline{\mathbb{Q}}$ corresponding to $X \rightarrow X(1)$. The Belyi 
degree of $Y$ is bounded from above by the index $d$ of $\Gamma$ in $\mathrm{SL}_{2}(\mathbb{Z})$. In particular,

$$
\max \left(h_{\mathrm{Fal}}(Y), e(Y), \Delta(Y),\left|\delta_{\mathrm{Fal}}(Y)\right|\right) \leq 10^{9} d^{7} .
$$

Remark 1.5.3. Nonexplicit versions of Corollary 1.5 .1 were previously known for certain modular curves. Firstly, polynomial bounds for Arakelov invariants of $X_{0}(n)$ with $n$ squarefree were previously known; see [Ullmo 2000, Théorème 1.1 and Corollaire 1.3; Abbes and Ullmo 1997; Michel and Ullmo 1998, Théorème 1.1; Jorgenson and Kramer 2009]. The proofs of these results rely on the theory of modular curves. Also, similar results for Arakelov invariants of $X_{1}(n)$ with $n$ squarefree were shown in [Edixhoven and de Jong 2011a; Mayer 2012]. Bounds for the self-intersection of the dualising sheaf of a Fermat curve of prime exponent are given in [Curilla and Kühn 2009; Kühn 2013].

1.6. The Couveignes-Edixhoven-Bruin algorithm. Corollary 1.5.1 guarantees that, under the Riemann hypothesis for $\zeta$-functions of number fields, the CouveignesEdixhoven-Bruin algorithm to compute coefficients of modular forms runs in polynomial time; see Theorem 5.0.1 for a more precise statement.

Conventions. By log, we mean the principal value of the natural logarithm. We define the maximum of the empty set and the product taken over the empty set as 1 .

\section{Arakelov geometry of curves over number fields}

We are going to apply Arakelov theory to smooth projective geometrically connected curves $X$ over number fields $K$. Arakelov [1974] defined an intersection theory on the arithmetic surfaces attached to such curves. Faltings [1984] extended Arakelov's work. In this section, we aim at giving the necessary definitions and results for what we need later (and we need at least to fix our notation).

We start with some preparations concerning Riemann surfaces and arithmetic surfaces. In Section 2.3, we define the (stable) Arakelov invariants of $X$ appearing in Theorem 1.1.1. Finally, we prove bounds for Arakelov invariants of $X$ in the height and the Arakelov norm of the Wronskian differential of a non-Weierstrass point; see Theorem 2.4.1.

2.1. Arakelov invariants of Riemann surfaces. Let $X$ be a compact connected Riemann surface of genus $g \geq 1$. The space of holomorphic differentials $\mathrm{H}^{0}\left(X, \Omega_{X}^{1}\right)$ carries a natural hermitian inner product

$$
(\omega, \eta) \mapsto \frac{i}{2} \int_{X} \omega \wedge \bar{\eta} .
$$

For any orthonormal basis $\left(\omega_{1}, \ldots, \omega_{g}\right)$ with respect to this inner product, the Arakelov $(1,1)$-form is the smooth positive real-valued $(1,1)$-form $\mu$ on $X$ given by 
$\mu=(i / 2 g) \sum_{k=1}^{g} \omega_{k} \wedge \overline{\omega_{k}}$. Note that $\mu$ is independent of the choice of orthonormal basis. Moreover, $\int_{X} \mu=1$.

Let $\operatorname{gr}_{X}$ be the Arakelov-Green function on $(X \times X) \backslash \Delta$, where $\Delta \subset X \times X$ denotes the diagonal; see [Arakelov 1974], [de Jong 2005a], [Edixhoven and de Jong 2011b] or [Faltings 1984]. The Arakelov-Green functions determine certain metrics whose curvature forms are multiples of $\mu$, called admissible metrics, on all line bundles $O_{X}(D)$, where $D$ is a divisor on $X$, as well as on the holomorphic cotangent bundle $\Omega_{X}^{1}$. Explicitly, for $D=\sum_{P} D_{P} P$ a divisor on $X$, the metric $\|\cdot\|$ on $O_{X}(D)$ satisfies $\log \|1\|(Q)=\operatorname{gr}_{X}(D, Q)$ for all $Q$ away from the support of $D$, where $\operatorname{gr}_{X}(D, Q):=\sum_{P} n_{P} \operatorname{gr}_{X}(P, Q)$. Furthermore, for a local coordinate $z$ at a point $a$ in $X$, the metric $\|\cdot\|_{\mathrm{Ar}}$ on the sheaf $\Omega_{X}^{1}$ satisfies

$$
-\log \|d z\|_{\operatorname{Ar}}(a)=\lim _{b \rightarrow a}\left(\operatorname{gr}_{X}(a, b)-\log |z(a)-z(b)|\right) .
$$

We will work with these metrics on $\mathscr{O}_{X}(P)$ and $\Omega_{X}^{1}$ (as well as on tensor product combinations of them) and refer to them as Arakelov metrics. A metrised line bundle $\mathscr{L}$ is called admissible if, up to a constant scaling factor, it is isomorphic to one of the admissible bundles $O_{X}(D)$. The line bundle $\Omega_{X}^{1}$ endowed with the above metric is admissible; see [Arakelov 1974].

For any admissible line bundle $\mathscr{L}$, we endow the determinant of cohomology

$$
\lambda(\mathscr{L})=\operatorname{det} \mathrm{H}^{0}(X, \mathscr{L}) \otimes \operatorname{det} \mathrm{H}^{1}(X, \mathscr{L})^{\vee}
$$

of the underlying line bundle with the Faltings metric; see Theorem 1 of [Faltings 1984]. We normalise this metric so that the metric on $\lambda\left(\Omega_{X}^{1}\right)=\operatorname{det} \mathrm{H}^{0}\left(X, \Omega_{X}^{1}\right)$ is induced by the hermitian inner product on $\mathrm{H}^{0}\left(X, \Omega_{X}^{1}\right)$ given above.

Let $\mathbb{W}_{g}$ be the Siegel upper half-space of complex symmetric $g$-by- $g$ matrices with positive-definite imaginary part. Let $\tau$ in $\mathbb{H}_{g}$ be the period matrix attached to a symplectic basis of $\mathrm{H}_{1}(X, \mathbb{Z})$, and consider the analytic Jacobian $J_{\tau}(X)=$ $\mathbb{C}^{g} /\left(\mathbb{Z}^{g}+\tau \mathbb{Z}^{g}\right)$ attached to $\tau$. On $\mathbb{C}^{g}$, one has a theta function $\vartheta(z ; \tau)=\vartheta_{0,0}(z ; \tau)=$ $\sum_{n \in \mathbb{Z}^{g}} \exp \left(\pi i^{t} n \tau n+2 \pi i^{t} n z\right)$, giving rise to a reduced effective divisor $\Theta_{0}$ and a line bundle $\mathcal{O}\left(\Theta_{0}\right)$ on $J_{\tau}(X)$. The function $\vartheta$ is not well-defined on $J_{\tau}(X)$. Instead, we consider the function

$$
\|\vartheta\|(z ; \tau)=(\operatorname{det} \Im(\tau))^{1 / 4} \exp \left(-\pi^{t} y(\Im(\tau))^{-1} y\right)|\vartheta(z ; \tau)|
$$

with $y=\Im(z)$. One can check that $\|\vartheta\|$ descends to a function on $J_{\tau}(X)$. Now consider on the other hand the set $\operatorname{Pic}_{g-1}(X)$ of divisor classes of degree $g-1$ on $X$. It comes with a canonical subset $\Theta$ given by the classes of effective divisors and a canonical bijection $\operatorname{Pic}_{g-1}(X) \stackrel{\sim}{\longrightarrow} J_{\tau}(X)$ mapping $\Theta$ onto $\Theta_{0}$. As a result, we can equip $\operatorname{Pic}_{g-1}(X)$ with the structure of a compact complex manifold, together with a divisor $\Theta$ and a line bundle $O(\Theta)$. Note that we obtain $\|\vartheta\|$ as a function 
on $\operatorname{Pic}_{g-1}(X)$. It can be checked that this function is independent of the choice of $\tau$. Furthermore, note that $\|\vartheta\|$ gives a canonical way to put a metric on the line bundle $\mathcal{O}(\Theta)$ on $\operatorname{Pic}_{g-1}(X)$.

For any line bundle $\mathscr{L}$ of degree $g-1$, there is a canonical isomorphism from $\lambda(\mathscr{L})$ to $\mathcal{O}(-\Theta)[\mathscr{L}]$, the fibre of $\mathcal{O}(-\Theta)$ at the point $[\mathscr{L}]$ in $\operatorname{Pic}_{g-1}(X)$ determined by $\mathscr{L}$. Faltings [1984, Section 3] proves that, when we give both sides the metrics discussed above, the norm of this isomorphism is a constant independent of $\mathscr{L}$. We will write this norm as $\exp \left(\delta_{\mathrm{Fal}}(X) / 8\right)$ and refer to $\delta_{\mathrm{Fal}}(X)$ as Faltings' delta invariant of $X$.

Let $S(X)$ be the invariant of $X$ defined in [de Jong 2005a, Definition 2.2]. More explicitly, by [de Jong 2005a, Theorem 2.5],

$$
\log S(X)=-\int_{X} \log \|\vartheta\|(g P-Q) \cdot \mu(P),
$$

where $Q$ is any point on $X$. It is related to Faltings' delta invariant $\delta_{\mathrm{Fal}}(X)$. In fact, let $\left(\omega_{1}, \ldots, \omega_{g}\right)$ be an orthonormal basis of $\mathrm{H}^{0}\left(X, \Omega_{X}^{1}\right)$. Let $b$ be a point on $X$, and let $z$ be a local coordinate about $b$. Write $\omega_{k}=f_{k} d z$ for $k=1, \ldots, g$. We have a holomorphic function

$$
W_{z}(\omega)=\operatorname{det}\left(\frac{1}{(l-1) !} \frac{d^{l-1} f_{k}}{d z^{l-1}}\right)_{1 \leq k, l \leq g}
$$

locally about $b$ from which we build the $g(g+1) / 2$-fold holomorphic differential $W_{z}(\omega)(d z)^{\otimes g(g+1) / 2}$. It is readily checked that this holomorphic differential is independent of the choice of local coordinate and orthonormal basis. Thus, the holomorphic differential $W_{z}(\omega)(d z)^{\otimes g(g+1) / 2}$ extends over $X$ to give a nonzero global section, denoted by $\mathrm{Wr}$, of the line bundle $\Omega_{X}^{\otimes g(g+1) / 2}$. The divisor of the nonzero global section $\mathrm{Wr}$, denoted by $\mathcal{W}$, is the divisor of Weierstrass points. This divisor is effective of degree $g^{3}-g$. We follow [de Jong 2005a, Definition 5.3] and denote the constant norm of the canonical isomorphism of (abstract) line bundles

$$
\Omega_{X}^{g(g+1) / 2} \otimes_{\mathcal{O}_{X}}\left(\Lambda^{g} \mathrm{H}^{0}\left(X, \Omega_{X}^{1}\right) \otimes_{\mathbb{C}} \mathscr{O}_{X}\right)^{\vee} \rightarrow \mathcal{O}_{X}(\mathscr{W})
$$

by $R(X)$. Then

$$
\log S(X)=\frac{1}{8} \delta_{\mathrm{Fal}}(X)+\log R(X) .
$$

Moreover, for any non-Weierstrass point $b$ in $X$,

$$
\operatorname{gr}_{X}(\mathcal{W}, b)-\log R(X)=\log \|\mathrm{Wr}\|_{\operatorname{Ar}}(b) .
$$

2.2. Arakelov's intersection pairing on an arithmetic surface. Let $K$ be a number field with ring of integers $O_{K}$, and let $S=\operatorname{Spec} O_{K}$. Let $p: \mathscr{X} \rightarrow S$ be an arithmetic surface, i.e., an integral regular flat projective $S$-scheme of relative dimension 1 with geometrically connected fibres. For the sake of clarity, let us note that $p: \mathscr{X} \rightarrow S$ is 
a regular projective model of the generic fibre $\mathscr{X}_{K} \rightarrow$ Spec $K$ in the sense of [Liu 2006a, Definition 10.1.1].

In this section, we will assume the genus of the generic fibre $\mathscr{X}_{K}$ to be positive. An Arakelov divisor $D$ on $\mathscr{L}$ is a divisor $D_{\text {fin }}$ on $\mathscr{X}$ plus a contribution $D_{\text {inf }}=\sum_{\sigma} \alpha_{\sigma} F_{\sigma}$ running over the embeddings $\sigma: K \rightarrow \mathbb{C}$ of $K$ into the complex numbers. Here the $\alpha_{\sigma}$ are real numbers and the $F_{\sigma}$ are formally the "fibres at infinity", corresponding to the Riemann surfaces $\mathscr{X}_{\sigma}$ associated to the algebraic curves $\mathscr{X} \times{ }_{O_{K}, \sigma} \mathbb{C}$. We let $\widehat{\operatorname{Div}}(\mathscr{X})$ denote the group of Arakelov divisors on $\mathscr{X}$. To a nonzero rational function $f$ on $\mathscr{X}$, we associate an Arakelov divisor $\widehat{\operatorname{div}}(f):=(f)_{\text {fin }}+(f)_{\text {inf }}$ with $(f)_{\text {fin }}$ the usual divisor associated to $f$ on $\mathscr{X}$ and $(f)_{\text {inf }}=\sum_{\sigma} v_{\sigma}(f) F_{\sigma}$, where $v_{\sigma}(f):=-\int_{\mathscr{X}_{\sigma}} \log |f|_{\sigma} \cdot \mu_{\sigma}$. Here $\mu_{\sigma}$ is the Arakelov $(1,1)$-form on $\mathscr{X}_{\sigma}$. We will say that two Arakelov divisors on $\mathscr{L}$ are linearly equivalent if their difference is of the form $\widehat{\operatorname{div}}(f)$ for some nonzero rational function $f$ on $\mathscr{X}$. We let $\widehat{\mathrm{Cl}}(\mathscr{X})$ denote the group of Arakelov divisors modulo linear equivalence on $\mathscr{X}$.

Arakelov [1974] showed that there exists a unique symmetric bilinear map $(\cdot, \cdot): \widehat{\mathrm{Cl}}(\mathscr{X}) \times \widehat{\mathrm{Cl}}(\mathscr{X}) \rightarrow \mathbb{R}$ with the following properties:

- If $D$ and $E$ are effective divisors on $\mathscr{X}$ without common component, then

$$
(D, E)=(D, E)_{\mathrm{fin}}-\sum_{\sigma: K \rightarrow \mathbb{C}} \operatorname{gr}_{\mathscr{X}_{\sigma}}\left(D_{\sigma}, E_{\sigma}\right),
$$

where $\sigma$ runs over the complex embeddings of $K$. Here $(D, E)_{\text {fin }}$ denotes the usual intersection number of $D$ and $E$ as in [Liu 2006a, Section 9.1]; i.e.,

$$
(D, E)_{\mathrm{fin}}=\sum_{s \in|S|} i_{s}(D, E) \log \# k(s),
$$

where $s$ runs over the set $|S|$ of closed points of $S, i_{s}(D, E)$ is the intersection multiplicity of $D$ and $E$ at $s$ and $k(s)$ denotes the residue field of $s$. Note that, if $D$ or $E$ is vertical, the sum $\sum_{\sigma: K \rightarrow \mathbb{C}} \operatorname{gr}_{\mathscr{X}_{\sigma}}\left(D_{\sigma}, E_{\sigma}\right)$ is zero.

- If $D$ is a horizontal divisor of generic degree $n$ over $S$, then $\left(D, F_{\sigma}\right)=n$ for every $\sigma: K \rightarrow \mathbb{C}$.

- If $\sigma_{1}, \sigma_{2}: K \rightarrow \mathbb{C}$ are complex embeddings, then $\left(F_{\sigma_{1}}, F_{\sigma_{2}}\right)=0$.

An admissible line bundle on $\mathscr{X}$ is the datum of a line bundle $\mathscr{L}$ on $\mathscr{L}$ together with admissible metrics on the restrictions $\mathscr{L}_{\sigma}$ of $\mathscr{L}$ to the $\mathscr{L}_{\sigma}$. Let $\widehat{\operatorname{Pic}}(\mathscr{L})$ denote the group of isomorphism classes of admissible line bundles on $\mathscr{X}$. To any Arakelov divisor $D=D_{\text {fin }}+D_{\text {inf }}$ with $D_{\text {inf }}=\sum_{\sigma} \alpha_{\sigma} F_{\sigma}$, we can associate an admissible line bundle $O_{\mathscr{X}}(D)$. In fact, for the underlying line bundle of $\mathcal{O}_{\mathscr{X}}(D)$, we take $\mathscr{O}_{\mathscr{X}}\left(D_{\text {fin }}\right)$. Then, we make this into an admissible line bundle by equipping the pull-back of $O_{\mathscr{X}}\left(D_{\mathrm{fin}}\right)$ to each $\mathscr{X}_{\sigma}$ with its Arakelov metric, multiplied by $\exp \left(-\alpha_{\sigma}\right)$. This 
induces an isomorphism

$$
\widehat{\mathrm{Cl}}(\mathscr{X}) \stackrel{\sim}{\longrightarrow} \widehat{\operatorname{Pic}}(\mathscr{X}) .
$$

In particular, the Arakelov intersection of two admissible line bundles on $\mathscr{X}$ is well-defined.

Recall that a metrised line bundle $(\mathscr{L},\|\cdot\|)$ on $\operatorname{Spec} O_{K}$ corresponds to an invertible $O_{K}$-module, $L$, say, with hermitian metrics on the $L_{\sigma}:=\mathbb{C} \otimes_{\sigma, O_{K}} L$. The Arakelov degree of $(\mathscr{L},\|\cdot\|)$ is the real number defined by

$$
\widehat{\operatorname{deg}}(\mathscr{L})=\widehat{\operatorname{deg}}(\mathscr{L},\|\cdot\|)=\log \#\left(L / O_{K} s\right)-\sum_{\sigma: K \rightarrow \mathbb{C}} \log \|s\|_{\sigma},
$$

where $s$ is any nonzero element of $L$ (independence of the choice of $s$ follows from the product formula).

Note that the relative dualising sheaf $\omega_{\mathscr{L} / O_{K}}$ of $p: \mathscr{X} \rightarrow S$ is an admissible line bundle on $\mathscr{X}$ if we endow the restrictions $\Omega_{\mathscr{X}_{\sigma}}^{1}$ of $\omega_{\mathscr{L} / O_{K}}$ to the $\mathscr{L}_{\sigma}$ with their Arakelov metric. Furthermore, for any section $P: S \rightarrow \mathscr{X}$, we have

$$
\widehat{\operatorname{deg}} P^{*} \omega_{\mathscr{X} / O_{K}}=\left(O_{X}(P), \omega_{\mathscr{X} / O_{K}}\right)=:\left(P, \omega_{\mathscr{X} / O_{K}}\right),
$$

where we endow the line bundle $P^{*} \omega_{\mathscr{X} / O_{K}}$ on Spec $O_{K}$ with the pull-back metric.

Definition 2.2.1. We say that $\mathscr{X}$ is semistable (or nodal) over $S$ if every geometric fibre of $\mathscr{X}$ over $S$ is reduced and has only ordinary double singularities; see [Liu 2006a, Definition 10.3.1]. We say that $\mathscr{X}$ is (relatively) minimal if it does not contain any exceptional divisor; see [Liu 2006a, Definition 9.3.12].

Remark 2.2.2. Suppose that $\mathscr{X}$ is semistable over $S$ and minimal. The blowingup $\mathscr{Y} \rightarrow \mathscr{X}$ along a smooth closed point on $\mathscr{X}$ is semistable over $S$ but no longer minimal.

2.3. Arakelov invariants of curves. Let $X$ be a smooth projective connected curve over $\overline{\mathbb{Q}}$ of genus $g \geq 1$. Let $K$ be a number field such that $X$ has a semistable minimal regular model $p: \mathscr{X} \rightarrow$ Spec $O_{K}$; see Theorems 10.1.8, 10.3.34.a and 10.4.3 in [Liu 2006a]. (Note that we implicitly chose an embedding $K \rightarrow \overline{\mathbb{Q}}$.)

The Faltings delta invariant of $X$, denoted by $\delta_{\mathrm{Fal}}(X)$, is defined as

$$
\delta_{\mathrm{Fal}}(X)=\frac{1}{[K: \mathbb{Q}]} \sum_{\sigma: K \rightarrow \mathbb{C}} \delta_{\mathrm{Fal}}\left(\mathscr{W}_{\sigma}\right),
$$

where $\sigma$ runs over the complex embeddings of $K$ into $\mathbb{C}$. Similarly, we define

$$
\|\vartheta\|_{\max }(X)=\left(\prod_{\sigma: K \rightarrow \mathbb{C}} \max _{\operatorname{Pic}_{g-1}\left(\mathscr{D}_{\sigma}\right)}\|\vartheta\|\right)^{1 /[K: \mathbb{Q}]} .
$$


Moreover, we define

$$
R(X)=\left(\prod_{\sigma: K \rightarrow \mathbb{C}} R\left(\mathscr{X}_{\sigma}\right)\right)^{1 /[K: \mathbb{Q}]} \text { and } S(X)=\left(\prod_{\sigma: K \rightarrow \mathbb{C}} S\left(\mathscr{C}_{\sigma}\right)\right)^{1 /[K: \mathbb{Q}]} .
$$

The Faltings height of $X$ is defined by

$$
h_{\mathrm{Fal}}(X)=\frac{\widehat{\operatorname{deg}} \operatorname{det} p_{*} \omega_{\mathscr{X} / O_{K}}}{[K: \mathbb{Q}]}=\frac{\widehat{\operatorname{deg}} \operatorname{det} R p_{*} \mathcal{O}_{\mathscr{X}}}{[K: \mathbb{Q}]},
$$

where we endow the determinant of cohomology with the Faltings metric; see Section 2.1. Note that $h_{\mathrm{Fal}}(X)$ coincides with the stable Faltings height of the Jacobian of $\mathscr{L}_{K}$; see [Szpiro 1985b, Chapter I, Lemma 3.2.1]. Furthermore, we define the self-intersection of the dualising sheaf of $X$, denoted by $e(X)$, as

$$
e(X):=\frac{\left(\omega_{\mathscr{X} / O_{K}}, \omega_{\mathscr{X} / O_{K}}\right)}{[K: \mathbb{Q}]},
$$

where we use Arakelov's intersection pairing on the arithmetic surface $\mathscr{X} / O_{K}$. The discriminant of $X$, denoted by $\Delta(X)$, is defined as

$$
\Delta(X)=\frac{\sum_{\mathfrak{p} \subset O_{K}} \delta_{\mathfrak{p}} \log \# k(\mathfrak{p})}{[K: \mathbb{Q}]},
$$

where $\mathfrak{p}$ runs through the maximal ideals of $O_{K}$ and $\delta_{\mathfrak{p}}$ denotes the number of singularities in the geometric fibre of $p: \mathscr{X} \rightarrow \operatorname{Spec} O_{K}$ over $\mathfrak{p}$. These invariants of $X$ are well-defined; see [Moret-Bailly 1990, Section 5.4].

To bound the above Arakelov invariants, we introduce two functions on $X(\overline{\mathbb{Q}})$ : the height and the Arakelov norm of the Wronskian differential. More precisely, let $b \in X(\overline{\mathbb{Q}})$ and suppose that $b$ induces a section $P$ of $\mathscr{X}$ over $O_{K}$. Then we define the height of $b$, denoted by $h(b)$, to be

$$
h(b)=\frac{\widehat{\operatorname{deg}} P^{*} \omega_{\mathscr{X} / O_{K}}}{[K: \mathbb{Q}]}=\frac{\left(P, \omega_{\mathscr{X} / O_{K}}\right)}{[K: \mathbb{Q}]} .
$$

Note that the height of $b$ is the stable canonical height of a point, in the Arakelovtheoretic sense, with respect to the admissible line bundle $\omega_{\mathscr{X} / O_{K}}$. We define the Arakelov norm of the Wronskian differential at $b$ as

$$
\|\mathrm{Wr}\|_{\operatorname{Ar}}(b)=\left(\prod_{\sigma: K \rightarrow \mathbb{C}}\|\mathrm{Wr}\|_{\operatorname{Ar}}\left(b_{\sigma}\right)\right)^{1 /[K: \mathbb{Q}]} .
$$

These functions on $X(\overline{\mathbb{Q}})$ are well-defined; see [Moret-Bailly 1990, Section 5.4].

Changing the model for $X$ might change the height of a point. Let us show that the height of a point does not become smaller if we take another regular model over $O_{K}$. 
Lemma 2.3.1. Let $9 \mathrm{y} \rightarrow \operatorname{Spec} O_{K}$ be an arithmetic surface. Assume that 9 is a model for $\mathscr{L}_{K}$. If $Q$ denotes the section of 9 over $O_{K}$ induced by $b \in X(\overline{\mathbb{Q}})$, then

$$
h(b) \leq \frac{\left(Q, \omega_{0} / O_{K}\right)}{[K: \mathbb{Q}]} .
$$

Proof. By the minimality of $\mathscr{X}$, there is a unique birational morphism $\phi: \mathscr{Y} \rightarrow \mathscr{X}$; see [Liu 2006a, Corollary 9.3.24]. By the factorisation theorem, this morphism is made up of a finite sequence

$$
\mathscr{y}=\mathscr{Y}_{n} \stackrel{\phi_{n}}{\longrightarrow} \mathscr{Y}_{n-1} \stackrel{\phi_{n-1}}{\longrightarrow} \cdots \stackrel{\phi_{1}}{\longrightarrow} \mathscr{Y}_{0}=\mathscr{X}
$$

of blowing-ups along closed points; see [Liu 2006a, Theorem 9.2.2]. For $i=$ $1, \ldots, n$, let $E_{i} \subset \mathscr{Y}_{i}$ denote the exceptional divisor of $\phi_{i}$. Since the line bundles $\omega_{y_{i} / O_{K}}$ and $\phi_{i}^{*} \omega_{y_{i-1} / O_{K}}$ agree on $\mathscr{Y}_{i}-E_{i}$, there is an integer $a$ such that

$$
\omega \mathrm{og}_{i} / O_{K}=\phi_{i}^{*} \omega_{\mathrm{y}_{i-1} / O_{K}} \otimes \mathrm{Og}_{i} \mathcal{O}_{\mathrm{g}_{i}}\left(a E_{i}\right) .
$$

Applying the adjunction formula, we see that $a=1$. Since $\phi_{i}$ restricts to the identity morphism on the generic fibre, we have a canonical isomorphism of admissible line bundles

$$
\omega_{y_{i} / O_{K}}=\phi_{i}^{*} \omega_{y_{i-1} / O_{K}} \otimes_{\mathcal{O g}_{i}} \operatorname{Oog}_{i}\left(E_{i}\right)
$$

Let $Q_{i}$ denote the section of $\mathscr{Y}_{i}$ over $O_{K}$ induced by $b \in X(\overline{\mathbb{Q}})$. Then

$$
\begin{aligned}
\left(Q_{i}, \omega \mathrm{g}_{i} / O_{K}\right) & =\left(Q_{i}, \phi_{i}^{*} \omega \mathrm{g}_{i-1} / O_{K}\right)+\left(Q_{i}, E_{i}\right) \\
& \geq\left(Q_{i}, \phi_{i}^{*} \omega \mathrm{g}_{i-1} / O_{K}\right) \\
& =\left(Q_{i-1}, \omega \mathrm{g}_{i-1} / O_{K}\right),
\end{aligned}
$$

where we used the projection formula in the last equality. Therefore, we conclude that

$$
\left(Q, \omega_{\mathrm{y} / O_{K}}\right)=\left(Q_{n}, \omega_{\mathrm{y}_{n} / O_{K}}\right) \geq\left(Q_{0}, \omega_{\mathrm{g}_{0} / O_{K}}\right)=\left(P, \omega_{\mathscr{X} / O_{K}}\right)=h(b)[K: \mathbb{Q}] .
$$

2.4. Bounding Arakelov invariants in the height of a non-Weierstrass point. In this section, we prove bounds for Arakelov invariants of curves in the height of a non-Weierstrass point and the Arakelov norm of the Wronskian differential in this point.

Theorem 2.4.1. Let $X$ be a smooth projective connected curve over $\overline{\mathbb{Q}}$ of genus $g \geq 1$. Let $b \in X(\overline{\mathbb{Q}})$. Then

$$
\begin{aligned}
e(X) & \leq 4 g(g-1) h(b), \\
\delta_{\mathrm{Fal}}(X) & \geq-90 g^{3}-4 g(2 g-1)(g+1) h(b) .
\end{aligned}
$$


Suppose that $b$ is not a Weierstrass point. Then

$$
\begin{aligned}
h_{\mathrm{Fal}}(X) & \leq \frac{1}{2} g(g+1) h(b)+\log \|\mathrm{Wr}\|_{\mathrm{Ar}}(b), \\
\delta_{\mathrm{Fal}}(X) & \leq 6 g(g+1) h(b)+12 \log \|\mathrm{Wr}\|_{\mathrm{Ar}}(b)+4 g \log (2 \pi), \\
\Delta(X) & \leq 2 g(g+1)(4 g+1) h(b)+12 \log \|\mathrm{Wr}\|_{\mathrm{Ar}}(b)+93 g^{3} .
\end{aligned}
$$

This theorem is essential to the proof of Theorem 1.1.1 given in Section 4.5. We give a proof of Theorem 2.4.1 at the end of this section.

Lemma 2.4.2. For a smooth projective connected curve $X$ over $\overline{\mathbb{Q}}$ of genus $g \geq 1$,

$$
\log \|\vartheta\|_{\max }(X) \leq \frac{g}{4} \log \max \left(1, h_{\mathrm{Fal}}(X)\right)+\left(4 g^{3}+5 g+1\right) \log 2 .
$$

Proof. We kindly thank R. de Jong for sharing this proof with us. We follow the idea of [Graftieaux 2001, Section 2.3.2]; see also [David 1991, Appendice]. Let $\mathscr{F}_{g}$ be the Siegel fundamental domain of dimension $g$ in the Siegel upper half-space $\mathbb{H}_{g}$, i.e., the space of complex $(g \times g)$-matrices $\tau$ in $\mathbb{H}_{g}$ such that the following properties are satisfied. Firstly, for every element $u_{i j}$ of $u=\Re(\tau)$, we have $\left|u_{i j}\right| \leq \frac{1}{2}$. Secondly, for every $\gamma$ in $\operatorname{Sp}(2 g, \mathbb{Z})$, we have $\operatorname{det} \Im(\gamma \cdot \tau) \leq \operatorname{det} \Im(\tau)$, and finally, $\Im(\tau)$ is Minkowski-reduced; i.e., for all $\xi=\left(\xi_{1}, \ldots, \xi_{g}\right) \in \mathbb{Z}^{g}$ and for all $i$ such that $\xi_{i}, \ldots, \xi_{g}$ are nonzero, we have $\xi \Im(\tau)^{t} \xi \geq(\Im(\tau))_{i i}$ and, for all $1 \leq i \leq g-1$ we have $(\Im(\tau))_{i, i+1} \geq 0$. One can show that $\mathscr{F}_{g}$ contains a representative of each $\operatorname{Sp}(2 g, \mathbb{Z})$-orbit in $\mathbb{H}_{g}$.

Let $K$ be a number field such that $X$ has a model $X_{K}$ over $K$. For any embedding $\sigma: K \rightarrow \mathbb{C}$, let $\tau_{\sigma}$ be an element of $\mathscr{F}_{g}$ such that $\operatorname{Jac}\left(X_{K, \sigma}\right) \cong \mathbb{C}^{g} /\left(\tau_{\sigma} \mathbb{Z}^{g}+\mathbb{Z}^{g}\right)$ as principally polarised abelian varieties, the matrix of the Riemann form induced by the polarisation of $\operatorname{Jac}\left(X_{K, \sigma}\right)$ being $\Im\left(\tau_{\sigma}\right)^{-1}$ on the canonical basis of $\mathbb{C}^{g}$. By a result of Bost (see [Graftieaux 2001, Lemme 2.12] or [Pazuki 2012]), we have

$$
\frac{1}{[K: \mathbb{Q}]} \sum_{\sigma: K \rightarrow \mathbb{C}} \log \operatorname{det}\left(\Im\left(\tau_{\sigma}\right)\right) \leq g \log \max \left(1, h_{\mathrm{Fal}}(X)\right)+\left(2 g^{3}+2\right) \log (2) .
$$

Here we used that the Faltings height of $X$ equals the Faltings height of its Jacobian. Now, let $\vartheta(z ; \tau)$ be the Riemann theta function as in Section 2.1, where $\tau$ is in $\mathscr{F}_{g}$ and $z=x+i y$ is in $\mathbb{C}^{g}$ with $x, y \in \mathbb{R}^{g}$. Combining (5) with the upper bound

$$
\exp \left(-\pi^{t} y(\Im(\tau))^{-1} y\right)|\vartheta(z ; \tau)| \leq 2^{3 g^{3}+5 g}
$$

implies the result. Let us prove (6). Note that, if we write $y=\Im(z)=(\Im(\tau)) \cdot b$ for $b$ in $\mathbb{R}^{g}$,

$$
\exp \left(-\pi^{t} g(\Im(\tau))^{-1} y\right)|\vartheta(z ; \tau)| \leq \sum_{n \in \mathbb{Z}^{g}} \exp \left(-\pi^{t}(n+b)(\Im(\tau))(n+b)\right) .
$$


Since $\Im(\tau)$ is Minkowski reduced, we have ${ }^{t} m \Im(\tau) m \geq c(g) \sum_{i=1}^{g} m_{i}^{2}(\Im(\tau))_{i i}$ for all $m$ in $\mathbb{R}^{g}$. Here $c(g)=\left(4 / g^{3}\right)^{g-1}\left(\frac{3}{4}\right)^{g(g-1) / 2}$. Also, $(\Im(\tau))_{i i} \geq \sqrt{3} / 2$ for all $i=1, \ldots, g$ (see [Igusa 1972, Chapter V.4] for these facts). We deduce that

$$
\begin{aligned}
\sum_{n \in \mathbb{Z}^{g}} \exp \left(-\pi^{t}(n+b)(\Im(\tau))(n+b)\right) & \leq \sum_{n \in \mathbb{Z}^{g}} \exp \left(-\sum_{i=1}^{g} \pi c(g)\left(n_{i}+b_{i}\right)^{2}(\Im(\tau))_{i i}\right) \\
\leq & \prod_{i=1}^{g} \sum_{n_{i} \in \mathbb{Z}} \exp \left(-\pi c(g)\left(n_{i}+b_{i}\right)^{2}(\Im(\tau))_{i i}\right) \\
& \leq \prod_{i=1}^{g} \frac{2}{1-\exp \left(-\pi c(g)(\Im(\tau))_{i i}\right)} \leq 2^{g}\left(1+\frac{2}{\pi \sqrt{3} c(g)}\right)^{g} .
\end{aligned}
$$

This proves (6).

Lemma 2.4.3. Let $a \in \mathbb{R}_{>0}$ and $b \in \mathbb{R}_{\leq 1}$. Then, for all real numbers $x \geq b$, $x-a \log \max (1, x)=\frac{1}{2} x+\frac{1}{2}(x-2 a \log \max (1, x)) \geq \frac{1}{2} x+\min \left(\frac{1}{2} b, a-a \log (2 a)\right)$.

Proof. It suffices to prove that $x-2 a \log \max (1, x) \geq \min (b, 2 a-2 a \log (2 a))$ for all $x \geq b$. To prove this, let $x \geq b$. Then, if $2 a \leq 1$, we have $x-2 a \log \max (1, x) \geq$ $b \geq \min (b, 2 a-2 a \log (2 a))$. (To prove that $x-2 a \log \max (1, x) \geq b$, we may assume that $x \geq 1$. It is easy to show that $x-2 a \log x$ is a nondecreasing function for $x \geq 1$. Therefore, for all $x \geq 1$, we conclude that $x-2 a \log x \geq 1 \geq b$.) If $2 a>1$, the function $x-2 a \log (x)$ attains its minimum value at $x=2 a$ on the interval $[1, \infty)$.

Lemma 2.4.4 (Bost). Let $X$ be a smooth projective connected curve over $\overline{\mathbb{Q}}$ of genus $g \geq 1$. Then

$$
h_{\mathrm{Fal}}(X) \geq-\log (2 \pi) g
$$

Proof. See [Gaudron and Rémond 2011, Corollaire 8.4]. (Note that the Faltings height $h(X)$ utilised by Bost, Gaudron and Rémond is bigger than $h_{\mathrm{Fal}}(X)$ due to a difference in normalisation. In fact, we have $h(X)=h_{\mathrm{Fal}}(X)+g \log (\sqrt{\pi})$. In particular, the slightly stronger lower bound $h_{\mathrm{Fal}}(X) \geq-\log (\sqrt{2} \pi) g$ holds.)

Lemma 2.4.5. Let $X$ be a smooth projective connected curve over $\overline{\mathbb{Q}}$ of genus $g \geq 1$. Then

$$
\begin{aligned}
\log S(X) & +h_{\mathrm{Fal}}(X) \\
& \geq \frac{1}{2} h_{\mathrm{Fal}}(X)-\left(4 g^{3}+5 g+1\right) \log 2+\min \left(-\frac{g}{2} \log (2 \pi), \frac{g}{4}-\frac{g}{4} \log \left(\frac{g}{2}\right)\right) .
\end{aligned}
$$


Proof. By the explicit formula (2) for $S(X)$ in Section 2.1 and our bounds on theta functions (Lemma 2.4.2),

$$
\log S(X)+h_{\mathrm{Fal}}(X) \geq-\frac{g}{4} \log \max \left(1, h_{\mathrm{Fal}}(X)\right)-\left(4 g^{3}+5 g+1\right) \log 2+h_{\mathrm{Fal}}(X) .
$$

Since $h_{\mathrm{Fal}}(X) \geq-g \log (2 \pi)$, the statement follows from Lemma 2.4.3 (with $x=$ $h_{\mathrm{Fal}}(X), a=g / 4$ and $\left.b=-g \log (2 \pi)\right)$.

Lemma 2.4.6. Let $X$ be a smooth projective connected curve of genus $g \geq 2$ over $\overline{\mathbb{Q}}$. Then

$$
\frac{(2 g-1)(g+1)}{8(g-1)} e(X)+\frac{1}{8} \delta_{\mathrm{Fal}}(X) \geq \log S(X)+h_{\mathrm{Fal}}(X) .
$$

Proof. By [de Jong 2005a, Proposition 5.6],

$$
e(X) \geq \frac{8(g-1)}{(g+1)(2 g-1)}\left(\log R(X)+h_{\mathrm{Fal}}(X)\right) .
$$

Note that $\log R(X)=\log S(X)-\delta_{\mathrm{Fal}}(X) / 8$; see (3) in Section 2.1. This implies the inequality.

Lemma 2.4.7 (Noether formula). Let X be a smooth projective connected curve over $\overline{\mathbb{Q}}$ of genus $g \geq 1$. Then

$$
12 h_{\mathrm{Fal}}(X)=e(X)+\Delta(X)+\delta_{\mathrm{Fal}}(X)-4 g \log (2 \pi) .
$$

Proof. This is well-known; see [Faltings 1984, Theorem 6; Moret-Bailly 1989, Théorème 2.2].

Proposition 2.4.8. Let $X$ be a smooth projective connected curve of genus $g \geq 2$ over $\overline{\mathbb{Q}}$. Then

$$
\begin{aligned}
h_{\mathrm{Fal}}(X) & \leq \frac{(2 g-1)(g+1)}{4(g-1)} e(X)+\frac{1}{4} \delta_{\mathrm{Fal}}(X)+20 g^{3}, \\
-g \log (2 \pi) & \leq \frac{(2 g-1)(g+1)}{4(g-1)} e(X)+\frac{1}{4} \delta_{\mathrm{Fal}}(X)+20 g^{3}, \\
\Delta(X) & \leq \frac{3(2 g-1)(g+1)}{g-1} e(X)+2 \delta_{\mathrm{Fal}}(X)+248 g^{3} .
\end{aligned}
$$

Proof. Firstly, by Lemma 2.4.6,

$$
\frac{(2 g-1)(g+1)}{8(g-1)} e(X)+\frac{1}{8} \delta_{\mathrm{Fal}}(X) \geq \log S(X)+h_{\mathrm{Fal}}(X) .
$$

To obtain the upper bound for $h_{\mathrm{Fal}}(X)$, we proceed as follows. By Lemma 2.4.5, $\log S(X)+h_{\mathrm{Fal}}(X)$

$$
\geq \frac{1}{2} h_{\mathrm{Fal}}(X)-\left(4 g^{3}+5 g+1\right) \log 2+\min \left(-\frac{g}{2} \log (2 \pi), \frac{g}{4}-\frac{g}{4} \log \left(\frac{g}{2}\right)\right) .
$$


From these two inequalities, we deduce that

$$
\begin{aligned}
\frac{1}{2} h_{\mathrm{Fal}}(X) \leq \frac{(2 g-1)(g+1)}{8(g-1)} e(X)+\frac{1}{8} \delta_{\mathrm{Fal}}(X) & +\left(4 g^{3}+5 g+1\right) \log 2 \\
& +\max \left(\frac{g}{2} \log (2 \pi), \frac{g}{4} \log \left(\frac{g}{2}\right)-\frac{g}{4}\right) .
\end{aligned}
$$

Finally, it is straightforward to verify the inequality

$$
\left(4 g^{3}+5 g+1\right) \log 2+\max \left(\frac{g}{2} \log (2 \pi), \frac{g}{4} \log \left(\frac{g}{2}\right)-\frac{g}{4}\right) \leq 10 g^{3} .
$$

This concludes the proof of the upper bound for $h_{\mathrm{Fal}}(X)$.

The second inequality follows from the first inequality of the proposition and the lower bound $h_{\mathrm{Fal}}(X) \geq-g \log (2 \pi)$ of Bost (Lemma 2.4.4).

Finally, to obtain the upper bound of the proposition for the discriminant of $X$, we eliminate the Faltings height of $X$ in the first inequality using the Noether formula and obtain

$$
\Delta(X)+e(X)+\delta_{\mathrm{Fal}}(X)-4 g \log (2 \pi) \leq \frac{3(2 g-1)(g+1)}{(g-1)} e(X)+3 \delta_{\mathrm{Fal}}(X)+240 g^{3} .
$$

Faltings [1984, Theorem 5] showed that $e(X) \geq 0$. Therefore, we conclude that

$$
\Delta(X)+\delta_{\mathrm{Fal}}(X)-4 g \log (2 \pi) \leq \frac{3(2 g-1)(g+1)}{(g-1)} e(X)+3 \delta_{\mathrm{Fal}}(X)+240 g^{3} .
$$

We are now ready to prove Theorem 2.4.1.

Proof of Theorem 2.4.1. The proof is straightforward. The upper bound $e(X) \leq$ $4 g(g-1) h(b)$ is well-known; see [Faltings 1984, Theorem 5].

Let us prove the lower bound for $\delta_{\mathrm{Fal}}(X)$. If $g \geq 2$, the lower bound for $\delta_{\mathrm{Fal}}(X)$ can be deduced from the second inequality of Proposition 2.4.8 and the upper bound $e(X) \leq 4 g(g-1) h(b)$. When $g=1$, this follows from a result of Szpiro [de Jong 2005b, Proposition 7.2] and the nonnegativity of $h(b)$.

From now on, we suppose that $b$ is a non-Weierstrass point. The upper bound $h_{\text {Fal }}(X) \leq \frac{1}{2} g(g+1) h(b)+\log \|\mathrm{Wr}\|_{\text {Ar }}(b)$ follows from Theorem 5.9 in [de Jong 2005a] and (4) in Section 2.1.

We deduce the upper bound $\delta_{\text {Fal }}(X) \leq 6 g(g+1) h(b)+12 \log \|\mathrm{Wr}\|_{\mathrm{Ar}}(b)+$ $4 g \log (2 \pi)$ as follows. Since $e(X) \geq 0$ and $\Delta(X) \geq 0$, the Noether formula implies that

$$
\delta_{\mathrm{Fal}}(X) \leq 12 h_{\mathrm{Fal}}(X)+4 g \log (2 \pi) .
$$

Thus, the upper bound for $\delta_{\mathrm{Fal}}(X)$ follows from the upper bound for $h_{\mathrm{Fal}}(X)$.

The upper bound $\Delta(X) \leq 2 g(g+1)(4 g+1) h(b)+12 \log \|\mathrm{Wr}\|_{\mathrm{Ar}}(b)+93 g^{3}$ follows from the inequality $\Delta(X) \leq 12 h_{\mathrm{Fal}}(X)-\delta_{\mathrm{Fal}}(X)+4 g \log (2 \pi)$ and the 
preceding bounds. (One could also use the last inequality of Proposition 2.4.8 to obtain a similar result.)

\section{Bounds for Arakelov-Green functions of Belyi covers}

Our aim is to give explicit bounds for the Arakelov-Green function on a Belyi cover of $X(2)$. Such bounds have been obtained for certain Belyi covers using spectral methods in [Jorgenson and Kramer 2006]. The results in [loc. cit.] do not apply to our situation since the smallest positive eigenvalue of the Laplacian can go to zero in a tower of Belyi covers; see [Long 2008, Theorem 4].

Instead, we use a theorem of Merkl to prove explicit bounds for the ArakelovGreen function on a Belyi cover in Theorem 3.4.5. More precisely, we construct a "Merkl atlas" for an arbitrary Belyi cover. Our construction uses an explicit version of [Jorgenson and Kramer 2004] on the Arakelov $(1,1)$-form due to Bruin.

We use our results to estimate the Arakelov norm of the Wronskian differential in Proposition 3.5.1.

Merkl's theorem [2011, Theorem 10.1] was used to prove bounds for ArakelovGreen functions of the modular curve $X_{1}(5 p)$ in [Edixhoven and de Jong 2011a].

3.1. Merkl's theorem. Let $X$ be a compact connected Riemann surface of positive genus, and recall that $\mu$ denotes the Arakelov $(1,1)$-form on $X$.

Definition 3.1.1. A Merkl atlas for $X$ is a quadruple

$$
\left(\left\{\left(U_{j}, z_{j}\right)\right\}_{j=1}^{n}, r_{1}, M, c_{1}\right),
$$

where $\left\{\left(U_{j}, z_{j}\right)\right\}_{j=1}^{n}$ is a finite atlas for $X$ and $\frac{1}{2}<r_{1}<1, M \geq 1$ and $c_{1}>0$ are real numbers such that the following properties are satisfied:

(1) Each $z_{j} U_{j}$ is the open unit disc.

(2) The open sets $U_{j}^{r_{1}}:=\left\{x \in U_{j}:\left|z_{j}(x)\right|<r_{1}\right\}$ with $1 \leq j \leq n$ cover $X$.

(3) For all $1 \leq j, j^{\prime} \leq n$, the function $\left|d z_{j} / d z_{j^{\prime}}\right|$ on $U_{j} \cap U_{j^{\prime}}$ is bounded from above by $M$.

(4) For $1 \leq j \leq n$, write $\mu_{\mathrm{Ar}}=i F_{j} d z_{j} \wedge d \overline{z_{j}}$ on $U_{j}$. Then $0 \leq F_{j}(x) \leq c_{1}$ for all $x \in U_{j}$.

Given a Merkl atlas $\left(\left\{\left(U_{j}, z_{j}\right)\right\}_{j=1}^{n}, r_{1}, M, c_{1}\right)$ for $X$, the following result provides explicit bounds for Arakelov-Green functions in $n, r_{1}, M$ and $c_{1}$ :

Theorem 3.1.2 (Merkl). Let $\left(\left\{\left(U_{j}, z_{j}\right)\right\}_{j=1}^{n}, r_{1}, M, c_{1}\right)$ be a Merkl atlas for $X$. Then

$$
\sup _{(X \times X) \backslash \Delta} \operatorname{gr}_{X} \leq \frac{330 n}{\left(1-r_{1}\right)^{3 / 2}} \log \frac{1}{1-r_{1}}+13.2 n c_{1}+(n-1) \log M .
$$


Furthermore, for every index $j$ and all $x \neq y \in U_{j}^{r_{1}}$, we have $\left|\operatorname{gr}_{X}(x, y)-\log \right| z_{j}(x)-z_{j}(y)|| \leq \frac{330 n}{\left(1-r_{1}\right)^{3 / 2}} \log \frac{1}{1-r_{1}}+13.2 n c_{1}+(n-1) \log M$. Proof. Merkl [2011] proved this theorem without explicit constants and without the dependence on $r_{1}$. A proof of the theorem in a more explicit form was given by P. Bruin in his master's thesis. This proof is reproduced, with minor modifications, in the Appendix.

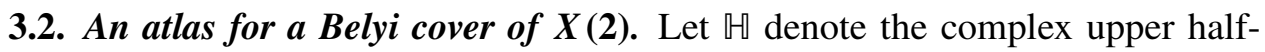
plane. Recall that $\mathrm{SL}_{2}(\mathbb{R})$ acts on $\mathbb{H}$ via Möbius transformations. Let $\Gamma(2)$ denote the subgroup of $\mathrm{SL}_{2}(\mathbb{Z})$ defined as

$$
\Gamma(2)=\left\{\left(\begin{array}{ll}
a & b \\
c & d
\end{array}\right) \in \mathrm{SL}_{2}(\mathbb{Z}): a \equiv d \equiv 1 \bmod 2 \text { and } b \equiv c \equiv 0 \bmod 2\right\} .
$$

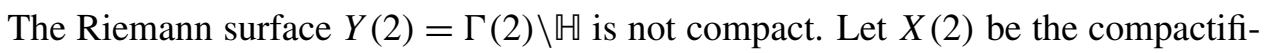

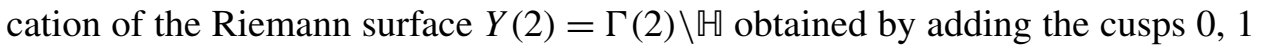
and $\infty$. Note that $X(2)$ is known as the compact modular curve associated to the congruence subgroup $\Gamma(2)$ of $\mathrm{SL}_{2}(\mathbb{Z})$. The modular lambda function $\lambda: \mathbb{H} \rightarrow \mathbb{C}$ induces an analytic isomorphism $\lambda: X(2) \rightarrow \mathbb{P}^{1}(\mathbb{C})$; see Section 4.4 for details. In particular, the genus of $X(2)$ is zero. For a cusp $\kappa \in\{0,1, \infty\}$, we fix an element $\gamma_{\kappa}$ in $\mathrm{SL}_{2}(\mathbb{Z})$ such that $\gamma_{\kappa}(\kappa)=\infty$.

We construct an atlas for the compact connected Riemann surface $X(2)$. Let $\dot{B}_{\infty}$ be the open subset given by the image of the strip

$$
\dot{S}_{\infty}:=\left\{x+i y:-1 \leq x<1, y>\frac{1}{2}\right\} \subset \mathbb{}
$$

in $Y(2)$ under the quotient map $\mathbb{H} \rightarrow \Gamma(2) \backslash \mathbb{W}$ defined by $\tau \mapsto \Gamma(2) \tau$. The quotient map $\mathbb{H} \rightarrow \Gamma(2) \backslash \mathbb{W}$ induces a bijection from this strip to $\dot{B}_{\infty}$. More precisely, suppose that $\tau$ and $\tau^{\prime}$ in $\dot{S}_{\infty}$ lie in the same orbit under the action of $\Gamma(2)$. Then, there exists an element

$$
\gamma=\left(\begin{array}{ll}
a & b \\
c & d
\end{array}\right) \in \Gamma(2)
$$

such that $\gamma \tau=\tau^{\prime}$. If $c \neq 0$, by definition, $c$ is a nonzero integral multiple of 2 . Thus, $c^{2} \geq 4$. Therefore,

$$
\frac{1}{2}<\Im \tau^{\prime}=\frac{\Im \tau}{|c \tau+d|^{2}} \leq \frac{1}{4 \Im \tau}<\frac{1}{2}
$$

This is clearly impossible. Thus, $c=0$ and $\tau^{\prime}=\tau \pm b$. By definition, $b=2 k$ for some integer $k$. Since $\tau$ and $\tau^{\prime}$ lie in the above strip, we conclude that $b=0$. Thus, $\tau=\tau^{\prime}$.

Consider the morphism $z_{\infty}: \mathbb{H} \rightarrow \mathbb{C}$ given by $\tau \mapsto \exp (\pi i \tau+\pi / 2)$. The image of the strip $\dot{S}_{\infty}$ under $z_{\infty}$ in $\mathbb{C}$ is the punctured open unit disc $\dot{B}(0,1)$. Now, for any $\tau$ 
and $\tau^{\prime}$ in the strip $\dot{S}_{\infty}$, the equality $z_{\infty}(\tau)=z_{\infty}\left(\tau^{\prime}\right)$ holds if and only if $\tau^{\prime}=\tau \pm 2 k$ for some integer $k$. But then $k=0$ and $\tau=\tau^{\prime}$. We conclude that $z_{\infty}$ factors injectively through $\dot{B}_{\infty}$. Let $z_{\infty}: B_{\infty} \rightarrow B(0,1)$ denote, by abuse of notation, the induced chart at $\infty$, where $B_{\infty}:=\dot{B}_{\infty} \cup\{\infty\}$ and $B(0,1)$ is the open unit disc in $\mathbb{C}$. We translate our neighbourhood $B_{\infty}$ at $\infty$ to a neighbourhood for $\kappa$, where $\kappa$ is a cusp of $X(2)$. More precisely, for any $\tau$ in $\mathbb{H}$, define $z_{\kappa}(\tau)=\exp \left(\pi i \gamma_{k}^{-1} \tau+\pi / 2\right)$. Let $\dot{B}_{\kappa}$ be the image of $\dot{S}_{\infty}$ under the map $\mathbb{H} \rightarrow Y(2)$ given by $\tau \mapsto \Gamma(2) \gamma_{\kappa} \tau$. We define $B_{\kappa}=\dot{B}_{\kappa} \cup\{\kappa\}$. We let $z_{\kappa}: B_{\kappa} \rightarrow B(0,1)$ denote the induced chart (by abuse of notation).

Since the open subsets $B_{\kappa}$ cover $X(2)$, we have constructed an atlas $\left\{\left(B_{\kappa}, z_{\kappa}\right)\right\}_{\kappa}$ for $X(2)$, where $\kappa$ runs through the cusps 0,1 and $\infty$.

Definition 3.2.1. A Belyi cover of $X(2)$ is a morphism of compact connected Riemann surfaces $Y \rightarrow X(2)$ that is unramified over $Y(2)$. The points of $Y$ not lying over $Y(2)$ are called cusps.

Lemma 3.2.2. Let $\pi: Y \rightarrow X(2)$ be a Belyi cover with $Y$ of genus $g$. Then, $g \leq \operatorname{deg} \pi$.

Proof. This is trivial for $g \leq 1$. For $g \geq 2$, the statement follows from the RiemannHurwitz formula.

Let $\pi: Y \rightarrow X(2)$ be a Belyi cover. We are going to "lift" the atlas $\left\{\left(B_{\kappa}, z_{\kappa}\right)\right\}$ for $X(2)$ to an atlas for $Y$.

Let $\kappa$ be a cusp of $X(2)$. The branched cover $\pi^{-1}\left(B_{\kappa}\right) \rightarrow B_{\kappa}$ restricts to a finite degree topological cover $\pi^{-1}\left(\dot{B}_{\kappa}\right) \rightarrow \dot{B}_{\kappa}$. In particular, the composed morphism

$$
\pi^{-1} \dot{B}_{\kappa} \rightarrow \dot{B}_{\kappa} \underset{\left.z_{\kappa}\right|_{\dot{B}_{\kappa}}}{\sim} \dot{B}(0,1)
$$

is a finite degree topological cover of $\dot{B}(0,1)$.

Recall that the fundamental group of $\dot{B}(0,1)$ is isomorphic to $\mathbb{Z}$. More precisely, for any connected topological cover of $V \rightarrow \dot{B}(0,1)$ of finite degree, there is a unique integer $e \geq 1$ such that $V \rightarrow \dot{B}(0,1)$ is isomorphic to the cover $\dot{B}(0,1) \rightarrow \dot{B}(0,1)$ given by $x \mapsto x^{e}$.

For every cusp $y$ of $Y$ lying over $\kappa$, let $\dot{V}_{y}$ be the unique connected component of $\pi^{-1} \dot{B}_{\kappa}$ whose closure $V_{y}$ in $\pi^{-1}\left(B_{\kappa}\right)$ contains $y$. Then, for any cusp $y$, there is a positive integer $e_{y}$ and an isomorphism $w_{y}: \dot{V}_{y} \stackrel{\sim}{\rightarrow} \dot{B}(0,1)$ such that $w_{y}^{e_{y}}=\left.z_{\kappa} \circ \pi\right|_{\dot{V}_{v}}$. The isomorphism $w_{y}: \dot{V}_{y} \rightarrow \dot{B}(0,1)$ extends to an isomorphism $w_{y}: V_{y} \rightarrow B(0,1)$ such that $w_{y}^{e_{y}}=\left.z_{\kappa} \circ \pi\right|_{V_{y}}$. This shows that $e_{y}$ is the ramification index of $y$ over $\kappa$. Note that we have constructed an atlas $\left\{\left(V_{y}, w_{y}\right)\right\}$ for $Y$, where $y$ runs over the cusps of $Y$. 
3.3. The Arakelov $(1,1)$-form and the hyperbolic metric. Let

$$
\mu_{\text {hyp }}(\tau)=\frac{i}{2} \frac{1}{\Im(\tau)^{2}} d \tau d \bar{\tau}
$$

be the hyperbolic metric on $\mathbb{H}$. A Fuchsian group is a discrete subgroup of $\mathrm{SL}_{2}(\mathbb{R})$. For any Fuchsian group $\Gamma$, the quotient space $\Gamma \backslash \mathbb{M}$ is a connected Hausdorff topological space and can be made into a Riemann surface in a natural way. The hyperbolic metric $\mu_{\text {hyp }}$ on $\mathbb{U}$ induces a measure on $\Gamma \backslash \mathbb{M}$, given by a smooth positive real-valued $(1,1)$-form outside the set of fixed points of elliptic elements of $\Gamma$. If the volume of $\Gamma \backslash \mathbb{W}$ with respect to this measure is finite, we call $\Gamma$ a cofinite Fuchsian group.

Let $\Gamma$ be a cofinite Fuchsian group, and let $X$ be the compactification of $\Gamma \backslash \mathbb{M}$ obtained by adding the cusps. We assume that $\Gamma$ has no elliptic elements and that the genus $g$ of $X$ is positive. There is a unique smooth function $F_{\Gamma}: X \rightarrow[0, \infty)$ that vanishes at the cusps of $\Gamma$ such that

$$
\mu=\frac{1}{g} F_{\Gamma} \mu_{\mathrm{hyp}} .
$$

A detailed description of $F_{\Gamma}$ is not necessary for our purposes.

Definition 3.3.1. Let $\pi: Y \rightarrow X(2)$ be a Belyi cover. Then we define the cofinite Fuchsian group $\Gamma_{Y}$ (or simply $\Gamma$ ) associated to $\pi: Y \rightarrow X(2)$ as follows. Since the topological fundamental group of $Y(2)$ equals $\Gamma(2) /\{ \pm 1\}$, we have $\pi^{-1}(Y(2))=$ $\Gamma^{\prime} \backslash \mathbb{W}$ for some subgroup $\Gamma^{\prime} \subset \Gamma(2) /\{ \pm 1\}$ of finite index. We define $\Gamma \subset \Gamma(2)$ to be the inverse image of $\Gamma^{\prime}$ under the quotient map $\Gamma(2) \rightarrow \Gamma(2) /\{ \pm 1\}$. Note that $\Gamma$ is a cofinite Fuchsian group without elliptic elements.

Theorem 3.3.2 (Jorgenson and Kramer). For any Belyi cover $\pi: Y \rightarrow X(2)$, where $Y$ has positive genus,

$$
\sup _{\tau \in Y} F_{\Gamma} \leq 64 \max _{y \in Y}\left(e_{y}\right)^{2} \leq 64(\operatorname{deg} \pi)^{2} .
$$

Proof. This is shown in [Bruin 2013]. More precisely, in the notation of [loc. cit.], Bruin shows that, with $a=1.44$, we have $N_{\mathrm{SL}_{2}(\mathbb{Z})}\left(z, 2 a^{2}-1\right) \leq 58$. In particular, $\sup _{z \in Y} N_{\Gamma}\left(z, z, 2 a^{2}-1\right) \leq 58$; see Section 8.2 in [loc. cit.]. Now, we apply Proposition 6.1 and Lemma 6.2 (with $\epsilon=2 \mathrm{deg} \pi$ ) in [loc. cit.] to deduce the sought inequality.

Remark 3.3.3. Jorgenson and Kramer [2004] prove a stronger (albeit nonexplicit) version of Theorem 3.3.2.

3.4. A Merkl atlas for a Belyi cover of $X(2)$. In this section, we prove bounds for Arakelov-Green functions of Belyi covers. 
Recall that we constructed an atlas $\left\{\left(B_{\kappa}, z_{\kappa}\right)\right\}_{\kappa}$ for $X(2)$. For a cusp $\kappa$ of $X(2)$, let

$$
y_{\kappa}: \mathbb{H} \rightarrow(0, \infty)
$$

be defined by $\tau \mapsto \Im\left(\gamma_{\kappa}^{-1} \tau\right)=\frac{1}{2}-\frac{\log \left|z_{\kappa}(\tau)\right|}{\pi}$. This induces a function $\dot{B}_{\kappa} \rightarrow(0, \infty)$, also denoted by $y_{\kappa}$.

Lemma 3.4.1. For any two cusps $\kappa$ and $\kappa^{\prime}$ of $X(2)$, we have

$$
\left|\frac{d z_{\kappa}}{d z_{\kappa^{\prime}}}\right| \leq 4 \exp (3 \pi / 2)
$$

on $B_{\kappa} \cap B_{\kappa^{\prime}}$.

Proof. We work on the complex upper half-plane $\mathbb{W}$. We may and do assume that $\kappa \neq \kappa^{\prime}$. By applying $\gamma_{\kappa^{\prime}}^{-1}$, we may and do assume that $\kappa^{\prime}=\infty$. On $B_{\kappa} \cap B_{\infty}$, we have $d z_{\kappa}(\tau)=\pi i \exp \left(\pi i \gamma_{\kappa}^{-1} \tau+\pi / 2\right) d\left(\gamma_{\kappa}^{-1} \tau\right)$ and $d z_{\infty}(\tau)=\pi i \exp (\pi i \tau+\pi / 2) d(\tau)$.

Therefore,

$$
\frac{d z_{\kappa}}{d z_{\infty}}(\tau)=\exp \left(\pi i\left(\gamma_{\kappa}^{-1} \tau-\tau\right)\right) \frac{d\left(\gamma_{\kappa}^{-1} \tau\right)}{d(\tau)}
$$

It follows from a simple calculation that, for $\gamma_{\kappa}^{-1}=\left(\begin{array}{ll}a & b \\ c & d\end{array}\right)$ with $c \neq 0$,

$$
\left|\frac{d z_{\kappa}}{d z_{\infty}}\right|(\tau)=\frac{1}{|c \tau+d|^{2}} \exp \left(\pi\left(y_{\infty}(\tau)-y_{\kappa}(\tau)\right)\right) .
$$

For $\tau$ and $\gamma_{\kappa}^{-1} \tau$ in $B_{\infty}$, one has $y_{\infty}(\tau)>\frac{1}{2}$ and $y_{\kappa}(\tau)>\frac{1}{2}$. From $|c \tau+d| \geq$ $y_{\infty}(\tau)=\Im(\tau)$, it follows that

$$
y_{\kappa}(\tau)=\Im\left(\gamma_{\kappa}^{-1}(\tau)\right)=\gamma_{\infty}\left(\frac{a \tau+b}{c \tau+d}\right)=\frac{\Im \tau}{|c \tau+d|^{2}} \leq \frac{\Im \tau}{(\Im \tau)^{2}} \leq 2,
$$

and similarly, $y_{\infty}(\tau) \leq 2$. The statement follows.

Let $\pi: Y \rightarrow X(2)$ be a Belyi cover, and let $V=\pi^{-1}(Y(2))$ be the complement of the set of cusps in $Y$. Recall that we constructed an atlas $\left\{\left(V_{y}, w_{y}\right)\right\}$ for $Y$. We assume that the genus $g$ of $Y$ is positive, and as usual, we let $\mu$ denote the Arakelov $(1,1)$-form on $Y$.

Lemma 3.4.2. Let $y$ be a cusp of $\pi: Y \rightarrow X(2)$ with $\kappa=\pi(y)$. Then

$$
i d w_{y} d \bar{w}_{y}=\frac{2 \pi^{2} y_{\kappa}^{2}\left|w_{y}\right|^{2}}{e_{y}^{2}} \mu_{\text {hyp }} \text { on } \dot{V}_{y} .
$$

Proof. Let $\kappa=\pi(y)$ in $X(2)$. We work on the complex upper half-plane. By the chain rule, we have

$$
d\left(z_{\kappa}\right)=d\left(w_{y}^{e_{y}}\right)=e_{y} w_{y}^{e_{y}-1} d w_{y} .
$$


Therefore,

$$
e_{y}^{2}\left|w_{y}\right|^{2 e_{y}-2} d w_{y} d \bar{w}_{y}=d z_{\kappa} d \bar{z}_{\kappa} .
$$

Note that $d z_{\kappa}=\pi i z_{\kappa} d\left(\gamma_{\kappa}^{-1}\right)$, where we view $\gamma_{\kappa}^{-1}: \mathbb{M} \rightarrow \mathbb{C}$ as a function. Therefore,

$$
e_{y}^{2}\left|w_{y}\right|^{2 e_{y}-2} d w_{y} d \bar{w}_{y}=\pi^{2}\left|z_{\kappa}\right|^{2} d\left(\gamma_{\kappa}^{-1}\right) d\left(\overline{\gamma_{\kappa}^{-1}}\right) .
$$

Since $\left|w_{y}^{e_{y}}\right|=\left|z_{\kappa}\right|$, we have

$$
\begin{aligned}
i d w_{y} d \bar{w}_{y} & =\frac{i \pi^{2}\left|w_{y}\right|^{2}}{e_{y}^{2}} d\left(\gamma_{\kappa}^{-1}\right) d\left(\overline{\gamma_{\kappa}^{-1}}\right) \\
& =\frac{2 \pi^{2} y_{\kappa}^{2}\left|w_{y}\right|^{2}}{e_{y}^{2}} \frac{i d\left(\gamma_{\kappa}^{-1}\right) d\left(\overline{\gamma_{\kappa}^{-1}}\right)}{2 y_{\kappa}^{2}}=\frac{2 \pi^{2} y_{\kappa}^{2}\left|w_{y}\right|^{2}}{e_{y}^{2}}\left(\mu_{\mathrm{hyp}} \circ \gamma_{\kappa}^{-1}\right) .
\end{aligned}
$$

Since $\mu_{\text {hyp }}$ is invariant under the action of $\mathrm{SL}_{2}(\mathbb{Z})$, this concludes the proof.

Proposition 3.4.3. Let $y$ be a cusp of $\pi: Y \rightarrow X(2)$. Write $\mu=i F_{y} d w_{y} d \bar{w}_{y}$ on $V_{y}$. Then $F_{y}$ is a subharmonic function on $V_{y}$ and

$$
0 \leq F_{y} \leq \frac{128 \exp (3 \pi)(\operatorname{deg} \pi)^{4}}{\pi^{2} g} .
$$

Proof. The first statement follows from [Jorgenson and Kramer 2004, p. 8]; see also [Bruin 2010, p. 58]. The lower bound for $F_{y}$ is clear from the definition. Let us prove the upper bound for $F_{y}$.

For a cusp $\kappa$ of $X(2)$, let $\dot{B}_{\kappa}(2) \subset \dot{B}_{\kappa}$ be the image of the strip $\{x+i y:-1 \leq x<1$, $y>2\}$ in $Y(2)$ under the map $\mathbb{H} \rightarrow Y(2)$ given by $\tau \mapsto \Gamma(2) \gamma_{\kappa} \tau$. For a cusp $y$ of $Y$ lying over $\kappa$, define $\dot{V}_{y}(2)=\pi^{-1}\left(\dot{B}_{\kappa}(2)\right)$ and $V_{y}(2)=\dot{V}_{y}(2) \cup\{y\}$. Since the boundary $\partial V_{y}(2)$ of $V_{y}(2)$ is contained in $V_{y}-V_{y}(2)$, by the maximum principle for subharmonic functions,

$$
\sup _{V_{y}} F_{y}=\max \left(\sup _{V_{y}(2)} F_{y}, \sup _{V_{y}-V_{y}(2)} F_{y}\right)=\max \left(\sup _{\partial V_{y}(2)} F_{y}, \sup _{V_{y}-V_{y}(2)} F_{y}\right)=\sup _{V_{y}-V_{y}(2)} F_{y} .
$$

By Lemma 3.4.2, Definition 3.3.1 and (7) in Section 3.3,

$$
F_{y}=F_{\Gamma} \frac{e_{y}^{2}}{2 g \pi^{2} y_{\kappa}^{2}\left|w_{y}\right|^{2}} .
$$

Note that $y_{\kappa}^{-2}<4$ on $V_{y}$. Furthermore,

$$
\sup _{V_{y}-V_{y}(2)}\left|w_{y}\right|^{-2} \leq \sup _{B_{\kappa}-B_{\kappa}(2)}\left|z_{\kappa}\right|^{-2}=\exp (-\pi) \sup _{B_{\kappa}-B_{\kappa}(2)} \exp \left(2 \pi y_{\kappa}\right) \leq \exp (3 \pi) .
$$

Thus, the proposition follows from Jorgenson-Kramer's upper bound for $F_{\Gamma}$ (Theorem 3.3.2). 
Definition 3.4.4. Define $s_{1}=\sqrt{1 / 2}$. Note that $\frac{1}{2}<s_{1}<1$. For any cusp $\kappa$ of $X(2)$, let $B_{\kappa}^{s_{1}}$ be the open subset of $B_{\kappa}$ whose image under $z_{\kappa}$ is $\left\{x \in \mathbb{C}:|x|<s_{1}\right\}$. Moreover, define the positive real number $r_{1}$ by the equation $r_{1}^{\operatorname{deg} \pi}=s_{1}$. Note that $\frac{1}{2}<r_{1}<1$. For all cusps $y$ of $\pi: Y \rightarrow X(2)$, define the subset $V_{y}^{r_{1}} \subset V_{y}$ by $V_{y}^{r_{1}}=\left\{x \in V_{y}:\left|w_{y}(x)\right|<r_{1}\right\}$.

Theorem 3.4.5. Let $\pi: Y \rightarrow X(2)$ be a Belyi cover such that $Y$ is of genus $g \geq 1$. Then

$$
\sup _{(Y \times Y) \backslash \Delta} \operatorname{gr}_{Y} \leq 6378027 \frac{(\operatorname{deg} \pi)^{5}}{g} .
$$

Moreover, for every cusp $y$ and all $x \neq x^{\prime}$ in $V_{y}^{r_{1}}$,

$$
\left|\operatorname{gr}_{Y}\left(x, x^{\prime}\right)-\log \right| w_{y}(x)-w_{y}\left(x^{\prime}\right)|| \leq 6378027 \frac{(\operatorname{deg} \pi)^{5}}{g} .
$$

Proof. Write $d=\operatorname{deg} \pi$. Let $s_{1}$ and $r_{1}$ be as in Definition 3.4.4. We define real numbers

$$
n:=\#(Y-V), \quad M:=4 d \exp (3 \pi) \quad \text { and } \quad c_{1}:=\frac{128 \exp (3 \pi) d^{4}}{\pi^{2} g} .
$$

Since $n$ is the number of cusps of $Y$, we have $n \leq 3 d$. Moreover,

$$
\frac{1}{1-r_{1}} \leq \frac{d}{1-s_{1}} \text {. }
$$

Note that

$$
\frac{330 n}{\left(1-r_{1}\right)^{3 / 2}} \log \frac{1}{1-r_{1}}+13.2 n c_{1}+(n-1) \log M \leq 6378027 \frac{d^{5}}{g} .
$$

Therefore, by Theorem 3.1.2, it suffices to show that

$$
\left(\left\{\left(V_{y}, w_{y}\right)\right\}_{y}, r_{1}, M, c_{1}\right),
$$

where $y$ runs over the cusps of $\pi: Y \rightarrow X(2)$, constitutes a Merkl atlas for $Y$.

The first condition of Merkl's theorem is satisfied. That is, $w_{y} V_{y}$ is the open unit disc in $\mathbb{C}$.

To verify the second condition of Merkl's theorem, we have to show that the open sets $V_{y}^{r_{1}}$ cover $Y$. For any $x \in V_{y}$, we have $x \in V_{y}^{r_{1}}$ if $\pi(x) \in B_{\kappa}^{s_{1}}$. In fact, for any $x$ in $V_{y}$, we have $\left|w_{y}(x)\right|<r_{1}$ if and only if

$$
\left|z_{\kappa}(\pi(x))\right|=\left|w_{y}(x)\right|^{e_{y}}<r_{1}^{e_{y}} .
$$

Since $r_{1}<1$, we see that $s_{1}=r_{1}^{d} \leq r_{1}^{e_{y}}$. Therefore, if $\pi(x)$ lies in $B_{\kappa}^{s_{1}}$, we see that $x$ lies in $V_{y}^{r_{1}}$. Now, since $s_{1}<\sqrt{3} / 2$, we have $X(2)=\bigcup_{\kappa \in[0,1, \infty]} B_{\kappa}^{s_{1}}$. We conclude that $Y=\bigcup_{y} V_{y}^{r_{1}}$, where $y$ runs through the cusps. 
Since we have already verified the fourth condition of Merkl's theorem in Proposition 3.4.3, it suffices to verify the third condition to finish the proof. Let $\kappa$ and $\kappa^{\prime}$ be cusps of $X(2)$. We may and do assume that $\kappa \neq \kappa^{\prime}$. Now, as usual, we work on the complex upper half-plane. By the chain rule,

$$
\left|\frac{d w_{y}}{d w_{y^{\prime}}}\right| \leq \frac{d}{\left|w_{y}\right|^{e_{y}-1}} \sup _{B_{\kappa} \cap B_{\kappa^{\prime}}}\left|\frac{d z_{\kappa}}{d z_{\kappa^{\prime}}}\right|
$$

on $V_{y} \cap V_{y^{\prime}}$. Note that $\left|w_{y}(\tau)\right|^{e_{y}-1} \geq\left|w_{y}(\tau)\right|^{e_{y}}=\left|z_{\kappa}(\tau)\right|$ for any $\tau$ in $\mathbb{H}$. Therefore,

$$
\left|\frac{d w_{y}}{d w_{y^{\prime}}}\right| \leq \frac{d}{\left|z_{\kappa}\right|} \sup _{B_{\kappa} \cap B_{\kappa^{\prime}}}\left|\frac{d z_{\kappa}}{d z_{\kappa^{\prime}}}\right| \leq M,
$$

where we used Lemma 3.4.1 and the inequality $\left|z_{\kappa}\right|>\exp (-3 \pi / 2)$ on $B_{\kappa} \cap B_{\kappa^{\prime}}$.

\subsection{The Arakelov norm of the Wronskian differential.}

Proposition 3.5.1. Let $\pi: Y \rightarrow X(2)$ be a Belyi cover with $Y$ of genus $g \geq 1$. Then

$$
\sup _{Y-\operatorname{Supp} \mathcal{W}} \log \|\mathrm{Wr}\|_{\mathrm{Ar}} \leq 6378028 g(\operatorname{deg} \pi)^{5} .
$$

Proof. Let $b$ be a non-Weierstrass point on $Y$, and let $y$ be a cusp of $Y$ such that $b$ lies in $V_{y}^{r_{1}}$. Let $\omega=\left(\omega_{1}, \ldots, \omega_{g}\right)$ be an orthonormal basis of $\mathrm{H}^{0}\left(Y, \Omega_{Y}^{1}\right)$. Then, as in Section 2.1,

$$
\log \|\mathrm{Wr}\|_{\mathrm{Ar}}(b)=\log \left|W_{w_{y}}(\omega)(b)\right|+\frac{g(g+1)}{2} \log \left\|d w_{y}\right\|_{\mathrm{Ar}}(b) .
$$

By Theorem 3.4.5,

$$
\frac{g(g+1)}{2} \log \left\|d w_{y}\right\|_{\operatorname{Ar}}(b) \leq 6378027 g(\operatorname{deg} \pi)^{5} .
$$

Let us show that $\log \left|W_{w_{y}}(\omega)(b)\right| \leq g(\operatorname{deg} \pi)^{5}$. Write $\omega_{k}=f_{k} d w_{y}$ on $V_{y}$. Note that $\omega_{k} \wedge \overline{\omega_{k}}=\left|f_{k}\right|^{2} d w_{y} \wedge d \bar{w}_{y}$. Therefore,

$$
\mu=\frac{i}{2 g} \sum_{k=1}^{g} \omega_{k} \wedge \overline{\omega_{k}}=\frac{i}{2 g} \sum_{k=1}^{g}\left|f_{k}\right|^{2} d w_{y} \wedge d \bar{w}_{y} .
$$

We deduce that $\sum_{k=1}^{g}\left|f_{k}\right|^{2}=2 g F_{y}$, where $F_{y}$ is the unique function on $V_{y}$ such that $\mu=i F_{y} d w_{y} \wedge d \bar{w}_{y}$. By our upper bound for $F_{y}$ (Proposition 3.4.3), for any $j=1, \ldots, g$,

$$
\sup _{V_{y}}\left|f_{j}\right|^{2} \leq \sup _{V_{y}} \sum_{k=1}^{g}\left|f_{k}\right|^{2}=2 g F_{y} \leq \frac{256 \exp (3 \pi)(\operatorname{deg} \pi)^{4}}{\pi^{2}} .
$$


By Hadamard's inequality,

$$
\log \left|W_{w_{y}}(\omega)(b)\right| \leq \sum_{l=0}^{g-1} \log \left(\sum_{k=1}^{g}\left|\frac{d^{l} f_{k}}{d w_{y}^{l}}\right|^{2}(b)\right)^{1 / 2} .
$$

Let $r_{1}<r<1$ be some real number. By Cauchy's integral formula, for any $0 \leq l \leq g-1$,

$$
\begin{aligned}
\left|\frac{d^{l} f_{k}}{d w_{y}^{l}}\right|(b) & =\left|\frac{l !}{2 \pi i} \int_{\left|w_{y}\right|=r} \frac{f_{k}}{\left(w_{y}-w_{y}(b)\right)^{l+1}} d w_{y}\right| \\
& \leq \frac{l !}{\left(r-r_{1}\right)^{l+1}} \sup _{V_{y}}\left|f_{k}\right| \leq \frac{g !}{\left(1-r_{1}\right)^{g}} \sup _{V_{y}}\left|f_{k}\right| .
\end{aligned}
$$

By the preceding estimations, since $g ! \leq g^{g}$ and $1 /\left(1-r_{1}\right) \leq \operatorname{deg} \pi /\left(1-s_{1}\right)$, we obtain that

$\log \left|W_{w_{y}}(\omega)(b)\right|$

$$
\begin{aligned}
& \leq \sum_{l=0}^{g-1} \log \left(\frac{g !}{\left(1-r_{1}\right)^{g}}\left(\sum_{k=1}^{g} \sup _{V_{y}}\left|f_{k}\right|^{2}\right)^{1 / 2}\right) \\
& \leq \sum_{l=0}^{g-1} \log \left(\frac{g !}{\left(1-r_{1}\right)^{g}}\left(\sum_{k=1}^{g} \frac{256 \exp (3 \pi)(\operatorname{deg} \pi)^{4}}{\pi^{2}}\right)^{1 / 2}\right) \\
& =g \log (g !)+g^{2} \log \left(\frac{1}{1-r_{1}}\right)+\frac{g}{2} \log \left(\frac{256 g \exp (3 \pi)}{\pi^{2}}\right)+2 g \log (\operatorname{deg} \pi) \\
& \leq\left(4.5+\log \left(\frac{1}{1-s_{1}}\right)+\frac{1}{2} \log \left(\frac{256 \exp (3 \pi)}{\pi^{2}}\right)\right) g^{2} \log (\operatorname{deg} \pi) \\
& \leq 13 g(\operatorname{deg} \pi)^{2} .
\end{aligned}
$$

Since $g \geq 1$ and $\pi: Y \rightarrow X(2)$ is a Belyi cover, the inequality deg $\pi \geq 3$ holds. Thus,

$$
13 g(\operatorname{deg} \pi)^{2} \leq \frac{13 g(\operatorname{deg} \pi)^{5}}{27} \leq g(\operatorname{deg} \pi)^{5} .
$$

\section{Points of bounded height}

4.1. Lenstra's generalisation of Dedekind's discriminant bound. Let $A$ be a discrete valuation ring of characteristic 0 with fraction field $K$. Let $\operatorname{ord}_{A}$ denote the valuation on $A$. Let $L / K$ be a finite field extension of degree $n$, and let $B$ be the integral closure of $A$ in $L$. Note that $L / K$ is separable, and $B / A$ is finite; see [Serre 1979, Proposition I.4.8].

The inverse different $\mathfrak{D}_{B / A}^{-1}$ of $B$ over $A$ is the fractional ideal

$$
\{x \in L: \operatorname{Tr}(x B) \subset A\},
$$


where $\operatorname{Tr}$ is the trace of $L$ over $K$. The inverse of the inverse different, denoted by $\mathfrak{D}_{B / A}$, is the different of $B$ over $A$. Note that $\mathfrak{D}_{B / A}$ is actually an integral ideal of $L$.

The following proposition (which we would like to attribute to H. W. Lenstra, Jr.) is a generalisation of Dedekind's discriminant bound; see [Serre 1979, Proposition III.6.13].

Proposition 4.1.1 (H. W. Lenstra, Jr.). Suppose that B is a discrete valuation ring of ramification index e over $A$. Then, the valuation $r$ of the different ideal $\mathfrak{D}_{B / A}$ on $B$ satisfies the inequality

$$
r \leq e-1+e \cdot \operatorname{ord}_{A}(n) .
$$

Proof. Let $x$ be a uniformiser of $A$. Since $A$ is of characteristic 0 , we may define $y:=1 / n x$; note that $y$ is an element of $K$. The trace of $y$ (as an element of $L$ ) is $1 / x$. Since $1 / x$ is not in $A$, this implies that the inverse different $\mathfrak{D}_{B / A}^{-1}$ is strictly contained in the fractional ideal $y B$. (If not, since $A$ and $B$ are discrete valuation rings, we would have that $y B$ is strictly contained in the inverse different.) In particular, the different $\mathfrak{D}_{B / A}$ strictly contains the fractional ideal $(n x)$. Therefore, the valuation $\operatorname{ord}_{B}\left(\mathfrak{D}_{B / A}\right)$ on $B$ of $\mathfrak{D}_{B / A}$ is strictly less than the valuation of $n x$. Thus,

$$
\operatorname{ord}_{B}\left(\mathfrak{D}_{B / A}\right)<\operatorname{ord}_{B}(n x)=e \cdot \operatorname{ord}_{A}(n x)=e\left(\operatorname{ord}_{A}(n)+1\right)=e \cdot \operatorname{ord}_{A}(n)+e .
$$

This concludes the proof of the inequality.

Remark 4.1.2. If the extension of residue fields of $B / A$ is separable, Proposition 4.1.1 follows from the remark following Proposition III.6.13 in [Serre 1979]. (The result in that proposition was conjectured by Dedekind and proved by Hensel when $A=\mathbb{Z}$.) The reader will see that, in the proof of Proposition 4.2.4, we have to deal with imperfect residue fields.

Proposition 4.1.3. Suppose that the residue characteristic $p$ of $A$ is positive. Let $m$ be the biggest integer such that $p^{m} \leq n$. Then, for $\beta \subset B$ a maximal ideal of $B$ with ramification index $e_{\beta}$ over $A$, the valuation $r_{\beta}$ of the different ideal $\mathfrak{D}_{B / A}$ at $\beta$ satisfies the inequality

$$
r_{\beta} \leq e_{\beta}-1+e_{\beta} \cdot \operatorname{ord}_{A}\left(p^{m}\right) .
$$

Proof. To compute $r_{\beta}$, we localise $B$ at $\beta$ and then take the completions $\widehat{A}$ and $\widehat{B_{\beta}}$ of $A$ and $B_{\beta}$, respectively. Let $d$ be the degree of $\widehat{B_{\beta}}$ over $\widehat{A}$. Then, by Lenstra's result (Proposition 4.1.1), the inequality

$$
r_{\beta} \leq e_{\beta}-1+e_{\beta} \cdot \operatorname{ord}_{\widehat{A}}(d)
$$

holds. By definition, $\operatorname{ord}_{\widehat{A}}(d)=\operatorname{ord}_{A}(d) \leq \operatorname{ord}_{A}\left(p^{m}\right)$. This concludes the proof.

4.2. Covers of arithmetic surfaces with fixed branch locus. Let $K$ be a number field with ring of integers $O_{K}$, and let $S=\operatorname{Spec} O_{K}$. Let $D$ be a reduced effective divisor on $\mathscr{X}=\mathbb{P}_{S}^{1}$, and let $U$ denote the complement of the support of $D$ in $\mathscr{X}$. 
Let $y \rightarrow S$ be an integral normal two-dimensional flat projective $S$-scheme with geometrically connected fibres, and let $\pi: \mathscr{Y} \rightarrow \mathscr{X}$ be a finite surjective morphism of $S$-schemes that is étale over $U$. Let $\psi: y^{\prime} \rightarrow$ y be the minimal resolution of singularities [Liu 2006a, Proposition 9.3.32]. Note that we have the following diagram of morphisms:

$$
\mathscr{Y}^{\prime} \stackrel{\psi}{\longrightarrow} \mathscr{Y} \stackrel{\pi}{\longrightarrow} \mathscr{X} \rightarrow S .
$$

Consider the prime decomposition $D=\sum_{i \in I} D_{i}$, where $I$ is a finite-index set. Let $D_{i j}$ be an irreducible component of $\pi^{-1}(D)$ mapping onto $D_{i}$, where $j$ is in the index set $J_{i}$. We define $r_{i j}$ to be the valuation of the different ideal of $O_{g y, D_{i j}} / \mathcal{O}_{\mathscr{X}, D_{i}}$. We define the ramification divisor $R$ to be $\sum_{i \in I} \sum_{j \in J_{i}} r_{i j} D_{i j}$. We define $B:=\pi_{*} R$.

We apply [Liu 2006a, 6.4.26] to obtain that there exists a dualising sheaf $\omega_{\mathrm{y} / \mathrm{S}}$ for $\mathscr{Y} \rightarrow S$ and a dualising sheaf $\omega_{\pi}$ for $\pi: \mathscr{Y} \rightarrow \mathscr{X}$ such that the adjunction formula

$$
\omega_{\mathscr{y} / S}=\pi^{*} \omega_{\mathscr{L} / S} \otimes \omega_{\pi}
$$

holds. Since the local ring at the generic point of a divisor on $\mathscr{X}$ is of characteristic 0 , basic properties of the different ideal imply that $\omega_{\pi}$ is canonically isomorphic to the line bundle $\mathrm{O}_{9}(R)$. We deduce the Riemann-Hurwitz formula

$$
\omega_{\mathscr{y} / S}=\pi^{*} \omega_{\mathscr{X} / S} \otimes O_{\mathscr{y}}(R) .
$$

Let $K_{\mathscr{L}}=-2 \cdot[\infty]$ be the divisor defined by the tautological section of $\omega_{\mathscr{L} / O_{K}}$. Let $K_{\text {O }}$ ' denote the Cartier divisor on $\mathrm{Y}^{\prime}$ defined by the rational section $d(\pi \circ \psi)$ of $\omega_{0 y} / S$. We define the Cartier divisor $K_{\text {oy }}$ on 9 analogously; i.e., $K_{\text {gy }}$ is the Cartier divisor on 9 defined by $d \pi$. Note that $K_{\odot y}=\psi_{*} K_{y^{\prime}}$. Also, the Riemann-Hurwitz formula implies the following equality of Cartier divisors:

$$
K_{\mathscr{y}}=\pi^{*} K_{\mathscr{L}}+R \text {. }
$$

Let $E_{1}, \ldots, E_{s}$ be the exceptional components of $\psi: y^{\prime} \rightarrow \mathscr{Y}$. Note that the pull-back of the Cartier divisor $\psi^{*} K_{\text {oy }}$ coincides with $K_{\text {oy }}$ on

$$
y^{\prime}-\bigcup_{i=1}^{s} E_{i} \text {. }
$$

Therefore, there exist integers $c_{i}$ such that

$$
K_{y^{\prime}}=\psi^{*} K_{\text {甲y }}+\sum_{i=1}^{s} c_{i} E_{i},
$$

where this is an equality of Cartier divisors (not only modulo linear equivalence). Note that $\left(\psi^{*} K_{\text {वy }}, E_{i}\right)=0$ for all $i$. In fact, $K_{\text {gy }}$ is linearly equivalent to a Cartier divisor with support disjoint from the singular locus of 9 . 
Lemma 4.2.1. For all $i=1, \ldots, s$, we have $c_{i} \leq 0$.

Proof. We have the following local statement. Let $y$ be a singular point of $\mathscr{y}$, and let $E_{1}, \ldots, E_{r}$ be the exceptional components of $\psi$ lying over $y$. We define

$$
V_{+}=\sum_{\substack{i=1 \\ c_{i}>0}}^{r} c_{i} E_{i}
$$

as the sum on the $c_{i}>0$. To prove the lemma, it suffices to show that $V_{+}=0$. Since the intersection form on the exceptional locus of $y^{\prime} \rightarrow$ $y$ is negative-definite [Liu 2006a, Proposition 9.1.27], to prove $V_{+}=0$, it suffices to show that $\left(V_{+}, V_{+}\right) \geq 0$. Clearly, to prove the latter inequality, it suffices to show that, for all $i$ such that $c_{i}>0$, we have $\left(V_{+}, E_{i}\right) \geq 0$. To do this, fix $i \in\{1, \ldots, r\}$ with $c_{i}>0$. Since $\mathrm{y}^{\prime} \rightarrow$ $y$ is minimal, we have that $E_{i}$ is not a $(-1)$-curve. In particular, by the adjunction formula, the inequality $\left(K_{y^{\prime}}, E_{i}\right) \geq 0$ holds. We conclude that

$$
\left(V_{+}, E_{i}\right)=\left(K_{y^{\prime}}, E_{i}\right)-\sum_{\substack{j=1 \\ c_{j}<0}}^{r} c_{j}\left(E_{j}, E_{i}\right) \geq 0,
$$

where, in the last inequality, we used that, for all $j$ such that $c_{j}<0$, we have that $E_{j} \neq E_{i}$.

Proposition 4.2.2. Let $P^{\prime}: S \rightarrow y^{\prime}$ be a section, and let $Q: S \rightarrow \mathscr{X}$ be the induced section. If the image of $P^{\prime}$ is not contained in the support of $K_{y^{\prime}}$, then

$$
\left(K_{\mathrm{y}^{\prime}}, P^{\prime}\right)_{\mathrm{fin}} \leq(B, Q)_{\mathrm{fin}} .
$$

Proof. By the Riemann-Hurwitz formula, we have $K_{\mathscr{y}}=\pi^{*} K_{\mathscr{L}}+R$. Therefore, by Lemma 4.2.1, we get that

$$
\begin{aligned}
\left(K_{\mathscr{y}^{\prime}}, P^{\prime}\right)_{\mathrm{fin}} & =\left(\psi^{*} K_{\text {} y}+\sum c_{i} E_{i}, P^{\prime}\right)_{\mathrm{fin}} \\
& =\left(\psi^{*} \pi^{*} K_{\mathscr{L}}+\psi^{*} R+\sum_{i=1}^{s} c_{i} E_{i}, P^{\prime}\right)_{\mathrm{fin}} \\
& \leq\left(\psi^{*} \pi^{*} K_{\mathscr{X}}, P^{\prime}\right)_{\mathrm{fin}}+\left(\psi^{*} R, P^{\prime}\right)_{\mathrm{fin}} .
\end{aligned}
$$

Since the image of $P^{\prime}$ is not contained in the support of $K_{9 y^{\prime}}$, we can apply the projection formula for the composed morphism $\pi \circ \psi: \mathscr{Y}^{\prime} \rightarrow \mathscr{X}$ to $\left(\psi^{*} \pi^{*} K_{\mathscr{X}}, P^{\prime}\right)_{\text {fin }}$ and $\left(\psi^{*} R, P^{\prime}\right)_{\text {fin }}$; see [Liu 2006a, Section 9.2]. This gives

$$
\left(K_{\mathscr{y}^{\prime}}, P^{\prime}\right)_{\mathrm{fin}} \leq\left(\psi^{*} \pi^{*} K_{\mathscr{L}}, P^{\prime}\right)_{\mathrm{fin}}+\left(\psi^{*} R, P^{\prime}\right)_{\mathrm{fin}}=\left(K_{\mathscr{L}}, Q\right)_{\mathrm{fin}}+\left(\pi_{*} R, Q\right)_{\mathrm{fin}} .
$$

Since $K_{\mathscr{L}}=-2 \cdot[\infty]$, the inequality $\left(K_{\mathscr{L}}, Q\right)_{\text {fin }} \leq 0$ holds. By definition, $B=\pi_{*} R$. This concludes the proof. 
We introduce some notation. For $i$ in $I$ and $j$ in $J_{i}$, let $e_{i j}$ and $f_{i j}$ be the ramification index and residue degree of $\pi$ at the generic point of $D_{i j}$, respectively. Moreover, let $\mathfrak{p}_{i} \subset O_{K}$ be the maximal ideal corresponding to the image of $D_{i}$ in Spec $O_{K}$. Then, note that $e_{i j}$ is the multiplicity of $D_{i j}$ in the fibre of $9 y$ over $\mathfrak{p}_{i}$. Now, let $e_{\mathfrak{p}_{i}}$ and $f_{\mathfrak{p}_{i}}$ be the ramification index and residue degree of $\mathfrak{p}_{i}$ over $\mathbb{Z}$, respectively. Finally, let $p_{i}$ be the residue characteristic of the local ring at the generic point of $D_{i}$ and, if $p_{i}>0$, let $m_{i}$ be the biggest integer such that $p_{i}^{m_{i}} \leq \operatorname{deg} \pi$, i.e., $m_{i}=\left\lfloor\log (\operatorname{deg} \pi) / \log \left(p_{i}\right)\right\rfloor$.

Lemma 4.2.3. Let $i$ be in I such that $0<p_{i} \leq \operatorname{deg} \pi$. Then, for all $j$ in $J_{i}$,

$$
r_{i j} \leq 2 e_{i j} m_{i} e_{\mathfrak{p}_{i}}
$$

Proof. Let ord $D_{i}$ be the valuation on the local ring at the generic point of $D_{i}$. Then, by Proposition 4.1.3, the inequality

$$
r_{i j} \leq e_{i j}-1+e_{i j} \cdot \operatorname{ord}_{D_{i}}\left(p_{i}^{m_{i}}\right)
$$

holds. Note that $\operatorname{ord}_{D_{i}}\left(p_{i}^{m_{i}}\right)=m_{i} e_{\mathfrak{p}_{i}}$. Since $p_{i} \leq \operatorname{deg} \pi$, we have that $m_{i} \geq 1$. Therefore,

$$
r_{i j} \leq e_{i j}-1+e_{i j} m_{i} e_{\mathfrak{p}_{i}} \leq 2 e_{i j} m_{i} e_{\mathfrak{p}_{i}}
$$

Let us introduce a bit more notation. Let $I_{1}$ be the set of $i$ in $I$ such that $D_{i}$ is horizontal (i.e., $p_{i}=0$ ) or $p_{i}>\operatorname{deg} \pi$. Let $D_{1}=\sum_{i \in I_{1}} D_{i}$. We are now finally ready to combine our results to bound the "nonarchimedean" part of the height of a point.

Proposition 4.2.4. Let $P^{\prime}: S \rightarrow \mathscr{Y}^{\prime}$ be a section, and let $Q: S \rightarrow \mathscr{X}$ be the induced section. If the image of $P^{\prime}$ is not contained in the support of $K_{\mathrm{y}^{\prime}}$, then

$$
\left(K_{\mathrm{y}^{\prime}}, P^{\prime}\right)_{\mathrm{fin}} \leq \operatorname{deg} \pi\left(D_{1}, Q\right)_{\mathrm{fin}}+2(\operatorname{deg} \pi)^{2} \log (\operatorname{deg} \pi)[K: \mathbb{Q}] .
$$

Proof. Note that

$$
B=\sum_{i \in I}\left(\sum_{j \in J_{i}} r_{i j} f_{i j}\right) D_{i}
$$

Let $I_{2}$ be the complement of $I_{1}$ in $I$. Let $D_{2}=\sum_{i \in I_{2}} D_{i}$, and note that $D=D_{1}+D_{2}$. In particular,

$$
\begin{aligned}
(B, Q)_{\mathrm{fin}} & =\sum_{i \in I} \sum_{j \in J_{i}} r_{i j} f_{i j}\left(D_{i}, Q\right)_{\mathrm{fin}} \\
& =\sum_{i \in I_{1}} \sum_{j \in J_{i}} r_{i j} f_{i j}\left(D_{i}, Q\right)_{\mathrm{fin}}+\sum_{i \in I_{2}} \sum_{j \in J_{i}} r_{i j} f_{i j}\left(D_{i}, Q\right)_{\mathrm{fin}} .
\end{aligned}
$$


Note that, for all $i$ in $I_{1}$ and $j$ in $J_{i}$, the ramification of $D_{i j}$ over $D_{i}$ is tame; i.e., the equality $r_{i j}=e_{i j}-1$ holds. Note that, for all $i$ in $I$, we have $\sum_{j \in J_{i}} e_{i j} f_{i j}=\operatorname{deg} \pi$. Thus,

$$
\sum_{i \in I_{1}} \sum_{j \in J_{i}} r_{i j} f_{i j}\left(D_{i}, Q\right)_{\mathrm{fin}} \leq \sum_{i \in I_{1}} \sum_{j \in J_{i}} e_{i j} f_{i j}\left(D_{i}, Q\right)_{\mathrm{fin}}=\operatorname{deg} \pi\left(D_{1}, Q\right)_{\mathrm{fin}} .
$$

We claim that

$$
\sum_{i \in I_{2}} \sum_{j \in J_{i}} r_{i j} f_{i j}\left(D_{i}, Q\right)_{\mathrm{fin}} \leq 2(\operatorname{deg} \pi)^{2} \log (\operatorname{deg} \pi)[K: \mathbb{Q}] .
$$

In fact, since, for all $i$ in $I_{2}$ and $j$ in $J_{i}$, by Lemma 4.2.3, the inequality

$$
r_{i j} \leq 2 e_{i j} m_{i} e_{\mathfrak{p}_{i}}
$$

holds, we have that

$$
\begin{aligned}
\sum_{i \in I_{2}} \sum_{j \in J_{i}} r_{i j} f_{i j}\left(D_{i}, Q\right)_{\mathrm{fin}} & \leq 2 \sum_{i \in I_{2}} m_{i} e_{\mathfrak{p}_{i}}\left(D_{i}, Q\right)_{\mathrm{fin}}\left(\sum_{j \in J_{i}} e_{i j} f_{i j}\right) \\
& =2(\operatorname{deg} \pi) \sum_{i \in I_{2}} m_{i} e_{\mathfrak{p}_{i}}\left(D_{i}, Q\right)_{\mathrm{fin}} .
\end{aligned}
$$

Note that $\left(D_{i}, Q\right)=\log \left(\# k\left(\mathfrak{p}_{i}\right)\right)=f_{\mathfrak{p}_{i}} \log p_{i}$. We conclude that

$$
\begin{aligned}
\sum_{i \in I_{2}} m_{i} e_{\mathfrak{p}_{i}}\left(D_{i}, Q\right)_{\text {fin }} & =\sum_{p \text { prime }}\left(\sum_{i \in I_{2}, p_{i}=p} e_{\mathfrak{p}_{i}} f_{\mathfrak{p}_{i}}\right)\left\lfloor\frac{\log (\operatorname{deg} \pi)}{\log p}\right\rfloor \log (p) \\
& =[K: \mathbb{Q}] \sum_{\mathscr{X}_{p} \cap\left|D_{2}\right| \neq \varnothing}\left\lfloor\frac{\log (\operatorname{deg} \pi)}{\log p}\right\rfloor \log (p),
\end{aligned}
$$

where the last sum runs over all prime numbers $p$ such that the fibre $\mathscr{X}_{p}$ contains an irreducible component of the support of $D_{2}$. Thus,

$$
(B, Q)_{\text {fin }} \leq(\operatorname{deg} \pi)\left(D_{1}, Q\right)_{\text {fin }}+2(\operatorname{deg} \pi)[K: \mathbb{Q}] \sum_{\mathscr{X}_{p} \cap D_{2} \neq \varnothing}\left\lfloor\frac{\log (\operatorname{deg} \pi)}{\log p}\right\rfloor \log (p) .
$$

Note that

$$
\sum_{\mathscr{X}_{p} \cap D_{2} \neq \varnothing}\left\lfloor\frac{\log (\operatorname{deg} \pi)}{\log p}\right\rfloor \log (p) \leq \sum_{\mathscr{X}_{p} \cap D_{2} \neq \varnothing} \log (\operatorname{deg} \pi) \leq \operatorname{deg} \pi \log (\operatorname{deg} \pi),
$$

where we used that $\mathscr{X}_{p} \cap D_{2} \neq \varnothing$ implies that $p \leq \operatorname{deg} \pi$. In particular,

$$
(B, Q)_{\mathrm{fin}} \leq(\operatorname{deg} \pi)\left(D_{1}, Q\right)_{\mathrm{fin}}+2(\operatorname{deg} \pi)^{2} \log (\operatorname{deg} \pi)[K: \mathbb{Q}] .
$$


By Proposition 4.2.2, we conclude that

$$
\left(K_{y^{\prime}}, P^{\prime}\right)_{\text {fin }} \leq(\operatorname{deg} \pi)\left(D_{1}, Q\right)_{\text {fin }}+2(\operatorname{deg} \pi)^{2} \log (\operatorname{deg} \pi)[K: \mathbb{Q}] .
$$

4.3. Models of covers of curves. In this section, we give a general construction for a model of a cover of the projective line. Let $K$ be a number field with ring of integers $O_{K}$, and let $S=\operatorname{Spec} O_{K}$.

Proposition 4.3.1. Let $\mathrm{y} \rightarrow \operatorname{Spec} O_{K}$ be a flat projective morphism with geometrically connected fibres of dimension 1, where 9 is an integral normal scheme. Then, there exists a finite field extension $L / K$ such that the minimal resolution of singularities of the normalisation of $\mathrm{y} \times O_{K} O_{L}$ is semistable over $O_{L}$.

Proof. This follows from [Liu 2006b, Corollary 2.8].

The main result of this section reads as follows.

Theorem 4.3.2. Let $K$ be a number field, and let $Y$ be a smooth projective geometrically connected curve over $K$. Then, for any finite morphism $\pi_{K}: Y \rightarrow \mathbb{P}_{K}^{1}$, there exists a number field $L / K$ such that

- the normalisation $\pi:$ y $\rightarrow \mathbb{P}^{1}{ }_{O_{L}}$ of $\mathbb{P}^{1}{ }_{O_{L}}$ in the function field of $Y_{L}$ is finite flat surjective,

- the minimal resolution of singularities $\psi: y^{\prime} \rightarrow$ y is semistable over $O_{L}$ and

- each irreducible component of the vertical part of the branch locus of the finite flat morphism $\pi:$ y $\rightarrow \mathbb{P}_{O_{L}}^{1}$ is of characteristic less than or equal to deg $\pi$. (The characteristic of a prime divisor $D$ on $\mathbb{P}_{O_{L}}^{1}$ is the residue characteristic of the local ring at the generic point of $D$.)

Proof. By Proposition 4.3.1, there exists a finite field extension $L / K$ such that the minimal resolution of singularities $\psi: y^{\prime} \rightarrow$ $y$ of the normalisation of $\mathbb{P}^{1}{ }_{O_{L}}$ in the function field of $Y_{L}$ is semistable over $O_{L}$. Note that the finite morphism $\pi:$ y $\rightarrow \mathbb{P}_{O_{L}}^{1}$ is flat. (The source is normal of dimension 2, and the target is regular.) Moreover, since the fibres of $y^{\prime} \rightarrow$ Spec $O_{L}$ are reduced, the fibres of y over $O_{L}$ are reduced. Let $\mathfrak{p} \subset O_{L}$ be a maximal ideal of residue characteristic strictly bigger than deg $\pi$, and note that the ramification of $\pi: y \rightarrow \mathbb{P}^{1}{ }_{O_{L}}$ over (each prime divisor of $\mathbb{P}_{O_{L}}^{1}$ lying over) $\mathfrak{p}$ is tame. Since the fibres of $y \rightarrow \operatorname{Spec} O_{L}$ are reduced, we see that the finite morphism $\pi$ is unramified over $\mathfrak{p}$. In fact, since $\mathbb{P}_{O_{L}}^{1} \rightarrow$ Spec $O_{L}$ has reduced (even smooth) fibres, the valuation of the different ideal $\mathscr{D}_{\mathscr{O}_{D} / \mathscr{O}_{\pi(D)}}$ on $\mathscr{O}_{D}$ of an irreducible component $D$ of $\mathscr{Y}_{\mathfrak{p}}$ lying over $\pi(D)$ in $\mathscr{X}$ is precisely the multiplicity of $D$ in $\mathscr{Y}_{\mathfrak{p}}$. (Here we let $O_{D}$ denote the local ring at the generic point of $D$ and $\mathrm{O}_{\pi(D)}$ the local ring at the generic point of $\pi(D)$.) Thus, each irreducible component of the vertical part of the branch locus of $\pi:$ oy $\rightarrow \mathbb{P}^{1}{ }_{O_{L}}$ is of characteristic less or equal to $\operatorname{deg} \pi$. 
4.4. The modular lambda function. The modular function $\lambda: \mathbb{U} \rightarrow \mathbb{C}$ is defined as

$$
\lambda(\tau)=\frac{\wp\left(\frac{1}{2}+\frac{\tau}{2}\right)-\wp\left(\frac{\tau}{2}\right)}{\wp\left(\frac{\tau}{2}\right)-\wp\left(\frac{1}{2}\right)},
$$

where $\wp$ denotes the Weierstrass elliptic function for the lattice $\mathbb{Z}+\tau \mathbb{Z}$ in $\mathbb{C}$. The function $\lambda$ is $\Gamma$ (2)-invariant. More precisely, $\lambda$ factors through the $\Gamma(2)$-quotient map $\mathbb{H} \rightarrow Y(2)$ and an analytic isomorphism $Y(2) \stackrel{\sim}{\longrightarrow} \mathbb{C} \backslash\{0,1\}$. Thus, the modular function $\lambda$ induces an analytic isomorphism $X(2) \rightarrow \mathbb{P}^{1}(\mathbb{C})$. Let us note that $\lambda(i \infty)=0, \lambda(1)=\infty$ and $\lambda(0)=1$.

The restriction of $\lambda$ to the imaginary axis $\{i y: y>0\}$ in $\mathbb{W}$ induces a homeomorphism, also denoted by $\lambda$, from $\{i y: y>0\}$ to the open interval $(0,1)$ in $\mathbb{R}$. In fact, for $\alpha$ in the open interval $(0,1)$,

$$
\lambda^{-1}(\alpha)=i \frac{\mathrm{M}(1, \sqrt{\alpha})}{\mathrm{M}(1, \sqrt{1-\alpha})},
$$

where $\mathrm{M}$ denotes the arithmetic-geometric mean.

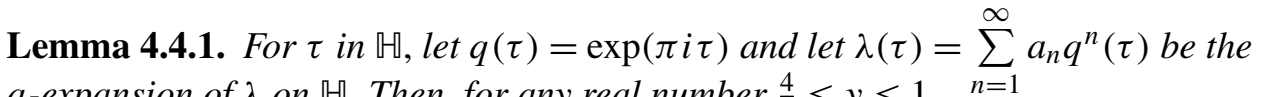
$q$-expansion of $\lambda$ on $\mathbb{H}$. Then, for any real number $\frac{4}{5} \leq y \leq 1$,

$$
-\log \left|\sum_{n=1}^{\infty} n a_{n} q^{n}(i y)\right| \leq 2 .
$$

Proof. Note that

$$
\sum_{n=1}^{\infty} n a_{n} q^{n}=q \frac{d \lambda}{d q}
$$

It suffices to show that $|q d \lambda / d q| \geq \frac{3}{20}$. We will use the product formula for $\lambda$. Namely,

$$
\lambda(q)=16 q \prod_{n=1}^{\infty} f_{n}(q) \quad \text { and } \quad f_{n}(q):=\frac{1+q^{2 n}}{1+q^{2 n-1}} .
$$

Write $f_{n}^{\prime}(q)=d f_{n}(q) / d q$. Then,

$$
q \frac{d \lambda}{d q}=\lambda\left(1+q \sum_{n=1}^{\infty} \frac{f_{n}^{\prime}(q)}{f_{n}(q)}\right)=\lambda\left(1+q \sum_{n=1}^{\infty} \frac{d}{d q} \log f_{n}(q)\right) .
$$

Note that, for any positive integer $n$ and $\frac{4}{5} \leq y \leq 1$,

$$
\left(\frac{d}{d q} \log f_{n}(q)\right)(i y) \leq 0 .
$$


Moreover, since $\lambda(i)=\frac{1}{2}$ and $\lambda(0)=1$, the inequality $\lambda(i y) \geq \frac{1}{2}$ holds for all $0 \leq y \leq 1$. Also, for $\frac{4}{5} \leq y \leq 1$,

$$
\left(-q \sum_{n=1}^{\infty} \frac{d}{d q} \log f_{n}(q)\right)(i y) \leq \frac{7}{10} .
$$

In fact,

$$
\sum_{n=1}^{\infty} \frac{d}{d q} \log f_{n}(q)=\sum_{n=1}^{\infty} \frac{2 n q^{2 n-1}}{1+q^{2 n}}-\sum_{n=1}^{\infty} \frac{(2 n-1) q^{2 n-2}}{1+q^{2 n-1}} .
$$

It is straightforward to verify that, for all $\frac{4}{5} \leq y \leq 1$, the inequality

$$
\begin{aligned}
\sum_{n=1}^{\infty} \frac{2 n q^{2 n-1}(i y)}{1+q^{2 n}(i y)}-\sum_{n=1}^{\infty} \frac{(2 n-1) q^{2 n-2}(i y)}{1+q^{2 n-1}(i y)} & \\
& \geq \frac{100}{109} \sum_{n=1}^{\infty} 2 n q^{2 n-1}(i y)-\sum_{n=1}^{\infty}(2 n-1) q^{2 n-2}(i y)
\end{aligned}
$$

holds. Finally, utilising classical formulas for geometric series, for all $\frac{4}{5} \leq y \leq 1$,

$$
q(i y) \sum_{n=1}^{\infty} \frac{d}{d q}\left(\log f_{n}(q)\right)(i y) \geq q(i y)\left(\frac{200 q(i y)}{109\left(1-q^{2}(i y)\right)^{2}}-\frac{1+q^{2}(i y)}{\left(1-q^{2}(i y)\right)^{2}}\right) \geq \frac{7}{10} .
$$

We conclude that

$$
\left|q \frac{d \lambda}{d q}\right| \geq \frac{1}{2}\left(1-\frac{7}{10}\right)=\frac{3}{20} .
$$

4.5. A non-Weierstrass point with bounded height. The logarithmic height of a nonzero rational number $a=p / q$ is given by

$$
h_{\text {naive }}(a)=\log \max (|p|,|q|),
$$

where $p$ and $q$ are coprime integers and $q>0$.

Theorem 4.5.1. Let $\pi_{\overline{\mathbb{Q}}}: Y \rightarrow \mathbb{P}_{\overline{\mathbb{Q}}}^{1}$ be a finite morphism of degree $d$, where $Y / \overline{\mathbb{Q}}$ is a smooth projective connected curve of positive genus $g \geq 1$. Assume that $\pi_{\overline{\mathbb{Q}}}: Y \rightarrow \mathbb{P}_{\overline{\mathbb{Q}}}^{1}$ is unramified over $\mathbb{P}_{\overline{\mathbb{Q}}}^{1} \backslash\{0,1, \infty\}$. Then, for any rational number $0<a \leq \frac{2}{3}$ and any $b \in Y(\overline{\mathbb{Q}})$ lying over $a$,

$$
h(b) \leq 3 h_{\text {naive }}(a) d^{2}+6378031 \frac{d^{5}}{g} .
$$

Proof. By Theorem 4.3.2, there exist a number field $K$ and a model

$$
\pi_{K}: Y \rightarrow \mathbb{P}_{K}^{1}
$$


for $\pi_{\overline{\mathbb{Q}}}: Y \rightarrow \mathbb{P}_{\overline{\mathbb{Q}}}^{1}$ with the following three properties: the minimal resolution of singularities $\psi: y^{\prime} \rightarrow \mathscr{Y}$ of the normalisation $\pi: \mathscr{Y} \rightarrow \mathbb{P}_{O_{K}}^{1}$ of $\mathbb{P}_{O_{K}}^{1}$ in $\mathscr{Y}$ is semistable over $O_{K}$, each irreducible component of the vertical part of the branch locus of $\pi: y \rightarrow \mathbb{P}^{1}{ }_{O_{K}}$ is of characteristic less than or equal to deg $\pi$ and every point in the fibre of $\pi_{K}$ over $a$ is $K$-rational. Also, the morphism $\pi: y \rightarrow \mathbb{P}^{1}{ }_{O_{K}}$ is finite flat surjective.

Let $b \in Y(K)$ lie over $a$. Let $P^{\prime}$ be the closure of $b$ in $\mathcal{Y}^{\prime}$. By Lemma 2.3.1, the height of $b$ is "minimal" on the minimal regular model. That is,

$$
h(b) \leq \frac{\left(P^{\prime}, \omega_{y^{\prime}} / O_{K}\right)}{[K: \mathbb{Q}]} .
$$

Recall the following notation from Section 4.2. Let $\mathscr{X}=\mathbb{P}_{O_{K}}^{1}$. Let $K_{\mathscr{X}}=-2 \cdot[\infty]$ be the divisor defined by the tautological section. Let $K_{\mathscr{y}} \mathrm{y}^{\prime}$ be the divisor on $\mathrm{Y}^{\prime}$ defined by $d\left(\pi_{K}\right)$ viewed as a rational section of $\omega_{0 y^{\prime} / O_{K}}$. Since the support of $K_{\triangle y^{\prime}}$ on the generic fibre is contained in $\pi_{K}^{-1}(\{0,1, \infty\})$, the section $P^{\prime}$ is not contained in the support of $K_{0 y^{\prime}}$. Therefore, we get that

$$
h(b)[K: \mathbb{Q}] \leq\left(P^{\prime}, \omega_{\mathrm{y}^{\prime}} / O_{K}\right)=\left(P^{\prime}, K_{\mathrm{y}^{\prime}}\right)_{\mathrm{fin}}+\sum_{\sigma: K \rightarrow \mathbb{C}}\left(-\log \left\|d \pi_{K}\right\|_{\sigma}\right)(\sigma(b)) .
$$

Let $D$ be the branch locus of $\pi: \mathscr{Y} \rightarrow \mathscr{X}$ endowed with the reduced closed subscheme structure. Write $D=0+1+\infty+D_{\text {ver }}$, where $D_{\text {ver }}$ is the vertical part of $D$. Note that, in the notation of Section 4.2, we have that $D_{1}=0+1+\infty$. Thus, if $Q$ denotes the closure of $a$ in $\mathscr{X}$, by Proposition 4.2.4, we get

$$
\left(P^{\prime}, K_{\text {Og' }}\right)_{\text {fin }} \leq(\operatorname{deg} \pi)(0+1+\infty, Q)_{\text {fin }}+2(\operatorname{deg} \pi)^{2} \log (\operatorname{deg} \pi)[K: \mathbb{Q}] .
$$

Write $a=p / q$, where $p$ and $q$ are coprime positive integers with $q>p$. Note that

$$
\begin{aligned}
(0+1+\infty, Q)_{\text {fin }} & =[K: \mathbb{Q}] \log (p q(q-p)) \\
& \leq 3 \log (q)[K: \mathbb{Q}] \\
& =3 h_{\text {naive }}(a)[K: \mathbb{Q}] .
\end{aligned}
$$

We conclude that

$$
\frac{\left(P^{\prime}, K_{\mathrm{y}^{\prime}}\right)_{\text {fin }}}{[K: \mathbb{Q}]} \leq 3 h_{\text {naive }}(a)(\operatorname{deg} \pi)^{2}+2(\operatorname{deg} \pi)^{3} .
$$

It remains to estimate $\sum_{\sigma: K \rightarrow \mathbb{C}}\left(-\log \left\|d \pi_{K}\right\|_{\sigma}\right)(\sigma(b))$. We will use our bounds for Arakelov-Green functions.

Let $\sigma: K \rightarrow \mathbb{C}$ be an embedding. The composition

$$
Y_{\sigma} \stackrel{\pi_{\sigma}}{\longrightarrow} \mathbb{P}^{1}(\mathbb{C}) \stackrel{\lambda^{-1}}{\longrightarrow} X(2)
$$


is a Belyi cover (Definition 3.2.1). By abuse of notation, let $\pi$ denote the composed morphism $Y_{\sigma} \rightarrow X(2)$. Note that $\lambda^{-1}\left(\frac{2}{3}\right) \approx 0.85 i$. In particular, $\Im\left(\lambda^{-1}(a)\right) \geq$ $\Im\left(\lambda^{-1}\left(\frac{2}{3}\right)\right)>s_{1}$. (Recall that $s_{1}=\sqrt{1 / 2}$.) Therefore, the element $\lambda^{-1}(a)$ lies in $\dot{B}_{\infty}^{s_{1}}$. Since $V_{y}^{r_{1}} \supset V_{y} \cap \pi^{-1} B_{\infty}^{s_{1}}$, there is a unique cusp $y$ of $Y_{\sigma} \rightarrow X(2)$ lying over $\infty$ such that $\sigma(b)$ lies in $V_{y}^{r_{1}}$.

Note that $q=z_{\infty} \exp (-\pi / 2)$. Therefore, since $\lambda=\sum_{j=1}^{\infty} a_{j} q^{j}$ on $\mathbb{H}$,

$$
\lambda \circ \pi=\sum_{j=1}^{\infty} a_{j} \exp (-j \pi / 2)\left(z_{\infty} \circ \pi\right)^{j}=\sum_{j=1}^{\infty} a_{j} \exp (-j \pi / 2) w_{y}^{e_{y} j}
$$

on $V_{y}$. Thus, by the chain rule,

$$
d(\lambda \circ \pi)=e_{y} \sum_{j=1}^{\infty} j a_{j} \exp (-j \pi / 2) w_{y}^{e_{y} j-1} d\left(w_{y}\right) .
$$

By the trivial inequality $e_{y} \geq 1$, the inequality $\left|w_{y}\right| \leq 1$ and Lemma 4.4.1, $-\log \|d(\lambda \circ \pi)\|_{\mathrm{Ar}}(\sigma(b))$

$$
\begin{aligned}
& =-\log \left\|d w_{y}\right\|_{\operatorname{Ar}}(\sigma(b))-\log \left|e_{y} \sum_{j=1}^{\infty} j a_{j} \exp (-j \pi / 2) w_{y}^{e_{y} j-1}(\sigma(b))\right| \\
& \leq-\log \left\|d w_{y}\right\|_{\operatorname{Ar}}(\sigma(b))-\log \left|\sum_{j=1}^{\infty} j a_{j} \exp (-j \pi / 2) w_{y}^{e_{y} j}(\sigma(b))\right| \\
& \leq-\log \left\|d w_{y}\right\|_{\mathrm{Ar}}(\sigma(b))+2 .
\end{aligned}
$$

Thus, by Theorem 3.4.5, we conclude that

$$
\frac{\sum_{\sigma: K \rightarrow \mathbb{C}}\left(-\log \left\|d \pi_{K}\right\|_{\sigma}\right)(\sigma(b))}{[K: \mathbb{Q}]} \leq 6378027 \frac{(\operatorname{deg} \pi)^{5}}{g}+2 .
$$

Theorem 4.5.2. Let $Y$ be a smooth projective connected curve over $\overline{\mathbb{Q}}$ of genus $g \geq 1$. For any finite morphism $\pi: Y \rightarrow \mathbb{P}_{\overline{\mathbb{Q}}}^{1}$ ramified over exactly three points, there exists a non-Weierstrass point $b$ on $Y$ such that

$$
h(b) \leq 6378033 \frac{(\operatorname{deg} \pi)^{5}}{g} .
$$

Proof. Define the sequence $\left(a_{n}\right)_{n=1}^{\infty}$ of rational numbers by $a_{1}=\frac{1}{2}$ and $a_{n}=$ $n /(2 n-1)$ for $n \geq 2$. Note that $\frac{1}{2} \leq a_{n} \leq \frac{2}{3}$ and that $h_{\text {naive }}\left(a_{n}\right) \leq \log (2 n)$. We may and do assume that $\pi: Y \rightarrow \mathbb{P}_{\overline{\mathbb{Q}}}^{1}$ is unramified over $\mathbb{P}_{\overline{\mathbb{Q}}}^{1} \backslash\{0,1, \infty\}$. By Theorem 4.5.1, for all $x \in \pi^{-1}\left(\left\{a_{n}\right\}\right)$,

$$
h(x) \leq 3 \log (2 n)(\operatorname{deg} \pi)^{2}+6378031 \frac{(\operatorname{deg} \pi)^{5}}{g} .
$$


Since the number of Weierstrass points on $Y$ is at most $g^{3}-g$, there exists an integer $1 \leq i \leq(\operatorname{deg} \pi)^{2}$ such that the fibre $\pi^{-1}\left(a_{i}\right)$ contains a non-Weierstrass point, say $b$. Applying (9) to $b$, we conclude that

$$
\begin{aligned}
h(b) & \leq 3 \log \left(2(\operatorname{deg} \pi)^{2}\right)(\operatorname{deg} \pi)^{2}+6378031 \frac{(\operatorname{deg} \pi)^{5}}{g} \\
& \leq 2 \frac{(\operatorname{deg} \pi)^{5}}{g}+6378031 \frac{(\operatorname{deg} \pi)^{5}}{g} .
\end{aligned}
$$

4.6. For a smooth projective connected curve $X$ over $\overline{\mathbb{Q}}$, we let $\operatorname{deg}_{B}(X)$ denote the Belyi degree of $X$.

Proof of Theorem 1.1.1. The inequality $\Delta(X) \geq 0$ is trivial, the lower bound $e(X) \geq 0$ is due to Faltings [1984, Theorem 5] and the lower bound $h_{\mathrm{Fal}}(X) \geq-g \log (2 \pi)$ is due to Bost (Lemma 2.4.4).

For the remaining bounds, we proceed as follows. By Theorem 4.5.2, there exists a non-Weierstrass point $b$ in $X(\overline{\mathbb{Q}})$ such that

$$
h(b) \leq 6378033 \frac{\operatorname{deg}_{B}(X)^{5}}{g} .
$$

By our bound on the Arakelov norm of the Wronskian differential in Proposition 3.5.1, we have $\log \|\mathrm{Wr}\|_{\mathrm{Ar}}(b) \leq 6378028 g \operatorname{deg}_{B}(X)^{5}$. To obtain the theorem, we combine these bounds with Theorem 2.4.1.

\section{Computing coefficients of modular forms}

Let $\Gamma \subset \mathrm{SL}_{2}(\mathbb{Z})$ be a congruence subgroup, and let $k$ be a positive integer. A modular form $f$ of weight $k$ for the group $\Gamma$ is determined by $k$ and its $q$-expansion coefficients $a_{m}(f)$ for $0 \leq m \leq k \cdot\left[\mathrm{SL}_{2}(\mathbb{Z}):\{ \pm 1\} \Gamma\right] / 12$. In this section, we follow [Bruin 2011] and give an algorithmic application of the main result of this paper. More precisely, the goal of this section is to complete the proof of the following theorem. The proof is given at the end of this section.

Theorem 5.0.1 (Couveignes, Edixhoven, Bruin). Assume the Riemann hypothesis for $\zeta$-functions of number fields. There exists a probabilistic algorithm that, given

- a positive integer $k$,

- a number field $K$,

- a congruence subgroup $\Gamma \subset \mathrm{SL}_{2}(\mathbb{Z})$,

- a modular form $f$ of weight $k$ for $\Gamma$ over $K$, and

- a positive integer $m$ in factored form,

computes $a_{m}(f)$ and whose expected running time is bounded by a polynomial in the length of the input. 
Remark 5.0.2. We should make precise how the number field $K$, the congruence subgroup $\Gamma$ and the modular form $f$ should be given to the algorithm and how the algorithm returns the coefficient $a_{m}(f)$. We should also explain what "probabilistic" means in this context. For the sake of brevity, we refer the reader to [Bruin 2011, p. 20] for the precise definitions. Following the definitions there, the above theorem becomes a precise statement.

Remark 5.0.3. The algorithm in Theorem 5.0.1 is due to Bruin, Couveignes and Edixhoven. Assuming the Riemann hypothesis for $\zeta$-functions of number fields, it was shown that the algorithm runs in polynomial time for certain congruence subgroups; see [Bruin 2011, Theorem 1.1]. Bruin did not have enough information about the semistable bad reduction of the modular curve $X_{1}(n)$ at primes $p$ such that $p^{2}$ divides $n$ to show that the algorithm runs in polynomial time. Nevertheless, our bounds on the discriminant of a curve can be used to show that the algorithm runs in polynomial time for all congruence subgroups.

Proof of Theorem 5.0.1. We follow Bruin's strategy [2010, Chapter V.1, p. 165]. He notes that, to assure that the algorithm runs in polynomial time for all congruence subgroups, it suffices to show that, for all positive integers $n$, the discriminant $\Delta\left(X_{1}(n)\right)$ is polynomial in $n$ (or equivalently the genus of $X_{1}(n)$ ). The latter follows from Corollary 1.5.1. In fact, the Belyi degree of $X_{1}(n)$ is at most the index of $\Gamma_{1}(n)$ in $\mathrm{SL}_{2}(\mathbb{Z})$. Since

$$
\left[\mathrm{SL}_{2}(\mathbb{Z}): \Gamma_{1}(n)\right]=n^{2} \prod_{p \mid n}\left(1-1 / p^{2}\right) \leq n^{2},
$$

we conclude that $\Delta\left(X_{1}(n)\right) \leq 5 \cdot 10^{8} n^{14}$.

\section{Bounds for heights of covers of curves}

Let $X$ be a smooth projective connected curve over $\overline{\mathbb{Q}}$. We prove that Arakelov invariants of (possibly ramified) covers of $X$ are polynomial in the degree. Let us be more precise.

Theorem 6.0.4. Let $X$ be a smooth projective connected curve over $\overline{\mathbb{Q}}$, let $U$ be a nonempty open subscheme of $X$, let $B_{f} \subset \mathbb{P}^{1}(\overline{\mathbb{Q}})$ be a finite set and let $f: X \rightarrow \mathbb{P}_{\overline{\mathbb{Q}}}^{1}$ be a finite morphism unramified over $\mathbb{P}_{\bar{Q}}^{1}-B_{f}$. Define

$$
B:=f(X \backslash U) \cup B_{f} .
$$

Let $N$ be the number of elements in the orbit of $B$ under the action of $\mathrm{Gal}(\overline{\mathbb{Q}} / \mathbb{Q})$, and let $H_{B}$ be the height of $B$ as defined in Section 1.3. Define

$$
c_{B}:=\left(4 N H_{B}\right)^{45 N^{3} 2^{N-2} N !} .
$$


Then, for any finite morphism $\pi: Y \rightarrow X$ étale over $U$, where $Y$ is a smooth projective connected curve over $\overline{\mathbb{Q}}$ of genus $g \geq 1$,

$$
\begin{aligned}
-\log (2 \pi) g & \leq h_{\mathrm{Fal}}(Y) \leq 13 \cdot 10^{6} g c_{B}(\operatorname{deg} f)^{5}(\operatorname{deg} \pi)^{5}, \\
0 & \leq e(Y) \leq 3 \cdot 10^{7}(g-1) c_{B}(\operatorname{deg} f)^{5}(\operatorname{deg} \pi)^{5}, \\
0 & \leq \Delta(Y) \leq 5 \cdot 10^{8} g^{2} c_{B}(\operatorname{deg} f)^{5}(\operatorname{deg} \pi)^{5}, \\
-10^{8} g^{2} c_{B}(\operatorname{deg} f)^{5}(\operatorname{deg} \pi)^{5} & \leq \delta_{\mathrm{Fal}}(Y) \leq 2 \cdot 10^{8} g c_{B}(\operatorname{deg} f)^{5}(\operatorname{deg} \pi)^{5} .
\end{aligned}
$$

Proof. We apply Khadjavi's effective version of Belyi's theorem. More precisely, by [Khadjavi 2002, Theorem 1.1.c], there exists a finite morphism $R: \mathbb{P}_{\overline{\mathbb{Q}}}^{1} \rightarrow \mathbb{P}_{\overline{\mathbb{Q}}}^{1}$ étale over $\mathbb{P}_{\bar{Q}}^{1} \backslash\{0,1, \infty\}$ such that $\operatorname{deg} R \leq\left(4 N H_{B}\right)^{9 N^{3} 2^{N-2} N !}$ and $R(B) \subset\{0,1, \infty\}$. Note that the composed morphism

$$
R \circ f \circ \pi: Y \stackrel{\pi}{\rightarrow} X \stackrel{f}{\rightarrow} \mathbb{P}_{\overline{\mathbb{Q}}}^{1} \stackrel{R}{\rightarrow} \mathbb{P}_{\overline{\mathbb{Q}}}^{1}
$$

is unramified over $\mathbb{P}_{\overline{\mathbb{Q}}}^{1} \backslash\{0,1, \infty\}$. We conclude by applying Theorem 1.1.1 to the composition $R \circ f \circ \pi$.

Note that Theorem 6.0.4 implies Theorem 1.3.1 (with $X=\mathbb{P}_{\overline{\mathbb{Q}}}^{1}, B_{f}$ the empty set and $f: X \rightarrow \mathbb{P}_{\overline{\mathbb{Q}}}^{1}$ the identity morphism).

In the proof of Theorem 6.0.4, we used Khadjavi's effective version of Belyi's theorem. Khadjavi's bounds are not optimal; see [Lițcanu 2004, Lemme 4.1] and [Khadjavi 2002, Theorem 1.1.b] for better bounds when $B$ is contained in $\mathbb{P}^{1}(\mathbb{Q})$. Actually, the use of Belyi's theorem makes the dependence on the branch locus enormous in Theorem 6.0.4. It should be possible to avoid the use of Belyi's theorem and improve the dependence on the branch locus in Theorem 6.0.4. This is not necessary for our present purposes.

Remark 6.0.5. Let us mention the quantitative Riemann existence theorem due to Bilu and Strambi [2010]. Bilu and Strambi give explicit bounds for the naive logarithmic height of a cover of $\mathbb{P}_{\overline{\mathbb{Q}}}^{1}$ with fixed branch locus. Although their bound on the naive height is exponential in the degree, the dependence on the height of the branch locus in their result is logarithmic.

Let us show that Theorem 1.3.1 implies the following:

Theorem 6.0.6 [Edixhoven et al. 2010, Conjecture 5.1]. Let $U \subset \mathbb{P}_{\mathbb{Z}}^{1}$ be a nonempty open subscheme. Then there are integers $a$ and $b$ with the following property. For any prime number $\ell$ and for any connected finite étale cover $\pi: V \rightarrow U_{\mathbb{Z}[1 / \ell]}$, the Faltings height of the normalisation of $\mathbb{P}_{\mathbb{Q}}^{1}$ in the function field of $V$ is bounded by $(\operatorname{deg} \pi)^{a} \ell^{b}$.

Proof. We claim that this conjecture holds with $b=0$ and an integer $a$ depending only on the generic fibre $U_{\mathbb{Q}}$ of $U$. In fact, let $\pi: Y \rightarrow \mathbb{P}_{\mathbb{Q}}^{1}$ denote the normalisation of $\mathbb{P}_{\mathbb{Q}}^{1}$ 
in the function field of $V$. Note that $\pi$ is étale over $U_{\mathbb{Q}}$. Let $B=\mathbb{P}_{\mathbb{Q}}^{1}-U_{\mathbb{Q}} \subset \mathbb{P}^{1}(\overline{\mathbb{Q}})$, and let $N$ be the number of elements in the orbit of $B$ under the action of $\operatorname{Gal}(\overline{\mathbb{Q}} / \mathbb{Q})$. By Theorem 1.3.1,

$$
h_{\mathrm{Fal}}(Y):=\sum_{X \subset Y_{\overline{\mathbb{Q}}}} h_{\mathrm{Fal}}(X) \leq(\operatorname{deg} \pi)^{a},
$$

where the sum runs over all connected components $X$ of $Y_{\overline{\mathbb{Q}}}:=Y \times_{\mathbb{Q}} \overline{\mathbb{Q}}$, and

$$
a=6+\log \left(13 \cdot 10^{6} N\left(4 N H_{B}\right)^{45 N^{3} 2^{N-2} N !}\right) .
$$

Here we used that $g \leq N \operatorname{deg} \pi$ and

$$
13 \cdot 10^{6} g\left(4 N H_{B}\right)^{45 N^{3} 2^{N-2} N !} \leq(\operatorname{deg} \pi)^{1+\log \left(13 \cdot 10^{6} N\left(4 N H_{B}\right)^{45 N^{3} 2^{N-2} N !}\right)} .
$$

This concludes the proof.

Let us briefly mention the context in which these results will hopefully be applied. Let $S$ be a smooth projective geometrically connected surface over $\mathbb{Q}$. As is explained in Section 5 of [Edixhoven et al. 2010], it seems reasonable to suspect that there exists an algorithm that, on input of a prime $\ell$, computes the étale cohomology groups $\mathrm{H}^{i}\left(S_{\overline{\mathbb{Q}}, \text { ét }}, \mathbb{F}_{\ell}\right)$ with their $\operatorname{Gal}(\overline{\mathbb{Q}} / \mathbb{Q})$-action in time polynomial in $\ell$ for all $i=0, \ldots, 4$.

\section{Appendix: Merkl's method of bounding Green functions}

by Peter Bruin

The goal of this appendix is to prove Theorem 3.1.2. Let $X$ be a compact connected Riemann surface, and let $\mu$ be a smooth nonnegative $(1,1)$-form on $X$ such that $\int_{X} \mu=1$. Let $*$ denote the star operator on 1 -forms on $X$, given with respect to a holomorphic coordinate $z=x+i y$ by

$$
* d x=d y \quad \text { and } \quad * d y=-d x,
$$

or equivalently

$$
* d z=-i d \bar{z} \quad \text { and } \quad * d \bar{z}=i d z
$$

The Green function for $\mu$ is the unique smooth function

$$
\operatorname{gr}_{\mu}:(X \times X) \backslash \Delta \rightarrow \mathbb{R},
$$

with a logarithmic singularity along the diagonal $\Delta$, such that for fixed $w \in X$ we have, in a distributional sense,

$$
\frac{1}{2 \pi} d * d \operatorname{gr}_{\mu}(z, w)=\delta_{w}(z)-\mu(z) \quad \text { and } \quad \int_{z \in X \backslash\{w\}} \operatorname{gr}_{\mu}(z, w) \mu(z)=0 .
$$


For all $a, b \in X$, we write $g_{a, b}$ for the unique smooth function on $X \backslash\{a, b\}$ satisfying

$$
d * d g_{a, b}=\delta_{a}-\delta_{b} \quad \text { and } \quad \int_{X \backslash\{a, b\}} g_{a, b} \mu=0 .
$$

Then for all $a \in X$, we consider the function $g_{a, \mu}$ on $X \backslash\{a\}$ defined by

$$
g_{a, \mu}(x)=\int_{b \in X \backslash\{x\}} g_{a, b}(x) \mu(b) .
$$

A straightforward computation using Fubini's theorem shows that this function satisfies

$$
d * d g_{a, \mu}=\delta_{a}-\mu \quad \text { and } \quad \int_{X \backslash\{a\}} g_{a, \mu} \mu=0 .
$$

This implies that $2 \pi g_{a, \mu}(b)=\operatorname{gr}_{\mu}(a, b)$, where $\operatorname{gr}_{\mu}$ is the Green function for $\mu$ defined above.

We begin by restricting our attention to one of the charts of our atlas, say $(U, z)$. By assumption, $z$ is an isomorphism from $U$ to the open unit disc in $\mathbb{C}$. Let $r_{2}$ and $r_{4}$ be real numbers with

$$
r_{1}<r_{2}<r_{4}<1
$$

and write

$$
r_{3}=\left(r_{2}+r_{4}\right) / 2
$$

We choose a smooth function

$$
\tilde{\chi}: \mathbb{R}_{\geq 0} \rightarrow[0,1]
$$

such that $\tilde{\chi}(r)=1$ for $r \leq r_{2}$ and $\tilde{\chi}(r)=0$ for $r \geq r_{4}$. We also define a smooth function $\chi$ on $X$ by putting

$$
\chi(x)=\tilde{\chi}(|z(x)|) \quad \text { for } x \in U
$$

and extending by 0 outside $U$. Furthermore, we put

$$
\chi^{c}=1-\chi .
$$

For $0<r<1$, we write

$$
U^{r}=\{x \in U:|z(x)|<r\} .
$$

For all $a, b \in U^{r_{1}}$, the function

$$
f_{a, b}=\frac{1}{2 \pi} \log \left|\frac{(z-z(a))\left(\overline{z(a)} z-r_{4}^{2}\right)}{(z-z(b))\left(\overline{z(b)} z-r_{4}^{2}\right)}\right|
$$


is defined on $U \backslash\{a, b\}$. Moreover, $f_{a, b}$ is harmonic on $U \backslash\{a, b\}$ since the logarithm of the modulus of a holomorphic function is harmonic. We extend $\chi^{\mathrm{c}} f_{a, b}$ to a smooth function on $U$ by defining it to be zero in $a$ and $b$.

We consider the open annulus

$$
A=U^{r_{4}} \backslash \overline{U^{r_{2}}} .
$$

Let $(\rho, \phi)$ be polar coordinates on $A$ such that $z=\rho \exp (i \phi)$. A straightforward calculation shows that in these coordinates the star operator is given by

$$
* d \rho=\rho d \phi \quad \text { and } \quad * d \phi=-\frac{d \rho}{\rho} .
$$

We consider the inner product

$$
\langle\alpha, \beta\rangle_{A}=\int_{A} \alpha \wedge * \beta
$$

on the $\mathbb{R}$-vector space of square-integrable real-valued 1-forms on A. Furthermore, we write

$$
\|\alpha\|_{A}^{2}=\langle\alpha, \alpha\rangle_{A} .
$$

Lemma A.1. For every real harmonic function $g$ on A such that $\|d g\|_{A}$ exists,

$$
\max _{|z|=r_{3}} g-\min _{|z|=r_{3}} g \leq \frac{2 \sqrt{\pi}}{r_{4}-r_{2}}\|d g\|_{A} .
$$

Proof. By the formula for the star operator in polar coordinates,

$$
\begin{aligned}
d g \wedge * d g & =\left(\partial_{\rho} g d \rho+\partial_{\phi} g d \phi\right) \wedge\left(\rho \partial_{\rho} g d \phi-\rho^{-1} \partial_{\phi} g d \rho\right) \\
& =\left(\left(\partial_{\rho} g\right)^{2}+\left(\rho^{-1} \partial_{\phi} g\right)^{2}\right) \rho d \rho d \phi .
\end{aligned}
$$

Using the mean value theorem, we can bound the left-hand side of the inequality we need to prove by

$$
\begin{aligned}
\max _{|z|=r_{3}} g-\min _{|z|=r_{3}} g & \leq \pi \max _{|z|=r_{3}}\left|\partial_{\phi} g\right| \\
& =\pi\left|\partial_{\phi} g\right|(x) \quad \text { for some } x \text { with }|z(x)|=r_{3} .
\end{aligned}
$$

We write $R=\left(r_{4}-r_{2}\right) / 2$, and we consider the open disc

$$
D=\{z \in U:|z-z(x)|<R\}
$$

of radius $R$ around $x$; this lies in $A$ because $r_{3}=\left(r_{4}+r_{2}\right) / 2$. Let $(\sigma, \psi)$ be polar coordinates on $D$ such that $z-z(x)=\sigma \exp (i \psi)$. Because $g$ is harmonic, so is $\partial_{\phi} g$, and Gauss's mean value theorem implies that

$$
\partial_{\phi} g(x)=\frac{1}{\pi R^{2}} \int_{D} \partial_{\phi} g \sigma d \sigma d \psi .
$$


On the space of real continuous functions on $D$, we have the inner product

$$
\left(h_{1}, h_{2}\right) \mapsto \int_{D} h_{1} h_{2} \sigma d \sigma d \psi
$$

Applying the Cauchy-Schwarz inequality with $h_{1}=\rho^{-1} \partial_{\phi} g$ and $h_{2}=\rho$ gives

$$
\begin{aligned}
\left|\int_{D} \partial_{\phi} g \sigma d \sigma d \psi\right| & \leq\left[\int_{D}\left(\rho^{-1} \partial_{\phi} g\right)^{2} \sigma d \sigma d \psi\right]^{1 / 2} \cdot\left[\int_{D} \rho^{2} \sigma d \sigma d \psi\right]^{1 / 2} \\
& \leq\left[\int_{A}\left(\rho^{-1} \partial_{\phi} g\right)^{2} \rho d \rho d \phi\right]^{1 / 2} \cdot\left[\int_{D} \sigma d \sigma d \psi\right]^{1 / 2} \\
& \leq\left[\int_{A} d g \wedge * d g\right]^{1 / 2}\left[\pi R^{2}\right]^{1 / 2} \\
& =\sqrt{\pi} R\|d g\|_{A} .
\end{aligned}
$$

Combining the above results finishes the proof.

Lemma A.2. For all $a, b \in U^{r_{1}}$, there exists a smooth function $\tilde{g}_{a, b}$ on $X$ such that

$$
d * d \tilde{g}_{a, b}= \begin{cases}d * d\left(\chi^{\mathrm{c}} f_{a, b}\right) & \text { on } U, \\ 0 & \text { on } X \backslash \bar{U} .\end{cases}
$$

It is unique up to an additive constant and fulfils

$$
\left\|d \tilde{g}_{a, b}\right\|_{A} \leq\left\|d\left(\chi^{\mathrm{c}} f_{a, b}\right)\right\|_{A} .
$$

Proof. First we note that the expression on the right-hand side of the equality defines a smooth 2-form on $X$ because $d * d\left(\chi^{\mathrm{c}} f_{a, b}\right)(z)$ vanishes for $|z|>r_{4}$; this follows from the choice of $\chi$ and the fact that $f_{a, b}$ is harmonic for $|z|>r_{1}$. Since moreover $\chi^{\mathrm{c}} f_{a, b}=0$ on $U^{r_{2}}$, we see that the support of this 2 -form is contained in the closed annulus $\bar{A}$. By Stokes's theorem,

$$
\int_{\bar{A}} d * d\left(\chi^{\mathrm{c}} f_{a, b}\right)=\int_{\partial \bar{A}} * d\left(\chi^{\mathrm{c}} f_{a, b}\right) .
$$

Notice that $f_{a, b}$ is invariant under the substitution $z \mapsto r_{4}^{2} / \bar{z}$; this implies that $\partial_{\rho} f_{a, b}(z)=0$ for $|z|=r_{4}$. Furthermore, $\chi^{\mathrm{c}}(z)=1$ and $d \chi^{\mathrm{c}}(z)=0$ for $|z|=r_{4}$, so we see that

$$
d\left(\chi^{\mathrm{c}} f_{a, b}\right)(z)=\chi^{\mathrm{c}}(z) d f_{a, b}(z)=\left(\partial_{\phi} f_{a, b} d \phi\right)(z) \quad \text { if }|z|=r_{4} .
$$

Likewise, since $\chi^{\mathrm{c}}=0$ and $d \chi^{\mathrm{c}}(z)=0$ for $|z|=r_{2}$,

$$
d\left(\chi^{\mathrm{c}} f_{a, b}\right)(z)=\chi^{\mathrm{c}}(z) d f_{a, b}(z)=0 \quad \text { if }|z|=r_{2} .
$$


This means that, for $z$ on the boundary of $\bar{A}$,

$$
* d\left(\chi^{\mathrm{c}} f_{a, b}\right)(z)= \begin{cases}-\left(\partial_{\phi} f_{a, b} d \rho\right)(z) & \text { if }|z|=r_{4}, \\ 0 & \text { if }|z|=r_{2} .\end{cases}
$$

In particular, $* d\left(\chi^{\mathrm{c}} f_{a, b}\right)$ vanishes when restricted to the submanifold $\partial \bar{A}$ of $X$. From this, we conclude that

$$
\int_{\bar{A}} d * d\left(\chi^{\mathrm{c}} f_{a, b}\right)=\int_{\partial \bar{A}} * d\left(\chi^{\mathrm{c}} f_{a, b}\right)=0 .
$$

This implies that a function $\tilde{g}_{a, b}$ with the required property exists.

To prove the inequality $\left\|d \tilde{g}_{a, b}\right\|_{A} \leq\left\|d\left(\chi^{\mathrm{c}} f_{a, b}\right)\right\|_{A}$, we note that

$$
\begin{aligned}
\left\|d\left(\chi^{\mathrm{c}} f_{a, b}\right)\right\|_{A}^{2} & =\left\|d \tilde{g}_{a, b}+d\left(\chi^{\mathrm{c}} f_{a, b}-\tilde{g}_{a, b}\right)\right\|_{A}^{2} \\
& =\left\|d \tilde{g}_{a, b}\right\|_{A}^{2}+2\left\langle d \tilde{g}_{a, b}, d\left(\chi^{\mathrm{c}} f_{a, b}-\tilde{g}_{a, b}\right)\right\rangle_{A}+\left\|d\left(\chi^{\mathrm{c}} f_{a, b}-\tilde{g}_{a, b}\right)\right\|_{A}^{2} .
\end{aligned}
$$

The last term is clearly nonnegative. Furthermore, integration by parts using Stokes's theorem gives

$$
\begin{aligned}
\left\langle d \tilde{g}_{a, b}, d\left(\chi^{\mathrm{c}} f_{a, b}-\tilde{g}_{a, b}\right)\right\rangle_{A} & =\int_{A} d \tilde{g}_{a, b} \wedge * d\left(\chi^{\mathrm{c}} f_{a, b}-\tilde{g}_{a, b}\right) \\
= & \int_{\partial \bar{A}} \tilde{g}_{a, b} * d\left(\chi^{\mathrm{c}} f_{a, b}-\tilde{g}_{a, b}\right)-\int_{A} \tilde{g}_{a, b} d * d\left(\chi^{\mathrm{c}} f_{a, b}-\tilde{g}_{a, b}\right) .
\end{aligned}
$$

The second term vanishes because $d * d \tilde{g}_{a, b}=d * d\left(\chi^{\mathrm{c}} f_{a, b}\right)$ on $A$. From our earlier expression for $* d\left(\chi^{\mathrm{c}} f_{a, b}\right)(z)$ on the boundary of $A$, we see that

$$
\int_{\partial \bar{A}} \tilde{g}_{a, b} * d\left(\chi^{\mathrm{c}} f_{a, b}\right)=0 .
$$

Finally, because $\partial \bar{A}$ is also the (negatively oriented) boundary of $X \backslash A$ and because $d * d \tilde{g}_{a, b}=0$ on $X \backslash A$,

$$
-\int_{\partial \bar{A}} \tilde{g}_{a, b} * d \tilde{g}_{a, b}=\int_{X \backslash A} d \tilde{g}_{a, b} \wedge * d \tilde{g}_{a, b} \geq 0 .
$$

Thus, we have

$$
\left\langle d \tilde{g}_{a, b}, d\left(\chi^{\mathrm{c}} f_{a, b}-\tilde{g}_{a, b}\right)\right\rangle_{A} \geq 0,
$$

which proves the inequality.

Lemma A.3. Let $\lambda=\max _{r_{2} \leq r \leq r_{4}}\left|\tilde{\chi}^{\prime}(r)\right|$. Then

$$
\max _{X} \tilde{g}_{a, b}-\min _{X} \tilde{g}_{a, b} \leq c_{3}\left(r_{1}, r_{2}, r_{4}, \lambda\right),
$$

where 
$c_{3}\left(r_{1}, r_{2}, r_{4}, \lambda\right)=$

$4 \sqrt{\frac{r_{4}+r_{2}}{r_{4}-r_{2}}}\left(\frac{\lambda}{2} \log \frac{\left(r_{1}+r_{4}\right)^{2}}{\left(r_{2}-r_{1}\right)\left(r_{4}-r_{1}\right)}+\frac{1}{r_{2}-r_{1}}+\frac{r_{1}}{r_{4}\left(r_{4}-r_{1}\right)}\right)+\frac{2}{\pi} \log \frac{\left(r_{1}+r_{4}\right)^{2}}{\left(r_{2}-r_{1}\right)\left(r_{4}-r_{1}\right)}$.

Proof. First, we note that

$$
\max _{X} \tilde{g}_{a, b}=\max \left\{\sup _{U^{r_{3}}} \tilde{g}_{a, b}, \sup _{X \backslash U^{r_{3}}} \tilde{g}_{a, b}\right\}, \quad \min _{X} \tilde{g}_{a, b}=\min \left\{\inf _{U^{r_{3}}} \tilde{g}_{a, b}, \inf _{X \backslash U^{r_{3}}} \tilde{g}_{a, b}\right\} .
$$

Furthermore,

$\sup _{U^{r_{3}}} \tilde{g}_{a, b} \leq \sup _{U^{r_{3}}}\left(\tilde{g}_{a, b}-\chi^{\mathrm{c}} f_{a, b}\right)+\sup _{U^{r_{3}}} \chi^{\mathrm{c}} f_{a, b}=\max _{|z|=r_{3}}\left(\tilde{g}_{a, b}-\chi^{\mathrm{c}} f_{a, b}\right)+\max _{r_{2} \leq|z| \leq r_{3}} \chi^{\mathrm{c}} f_{a, b}$

because of the maximum principle $\left(\tilde{g}_{a, b}-\chi^{\mathrm{c}} f_{a, b}\right.$ is harmonic on $\left.U\right)$ and because $\chi^{\mathrm{c}}(z)=0$ for $|z|<r_{2}$. In the same way, we find

$$
\inf _{U^{r_{3}}} \tilde{g}_{a, b} \geq \min _{|z|=r_{3}}\left(\tilde{g}_{a, b}-\chi^{\mathrm{c}} f_{a, b}\right)+\min _{r_{2} \leq|z| \leq r_{3}} \chi^{\mathrm{c}} f_{a, b} .
$$

We extend $\chi f_{a, b}$ to a smooth function on $X \backslash\{a, b\}$ by putting $\left(\chi f_{a, b}\right)(x)=0$ for $x \notin U$. Then $\tilde{g}_{a, b}+\chi f_{a, b}$ is harmonic on $X \backslash\{a, b\}$, and the same method as above gives us

$$
\begin{aligned}
\sup _{X \backslash U^{r_{3}}} \tilde{g}_{a, b} & \leq \max _{|z|=r_{3}}\left(\tilde{g}_{a, b}+\chi f_{a, b}\right)-\min _{r_{3} \leq|z| \leq r_{4}} \chi f_{a, b} \\
& \leq \max _{|z|=r_{3}}\left(\tilde{g}_{a, b}-\chi^{\mathrm{c}} f_{a, b}\right)+\max _{|z|=r_{3}} f_{a, b}-\min _{r_{3} \leq|z| \leq r_{4}} \chi f_{a, b}
\end{aligned}
$$

and

$$
\inf _{X \backslash U^{r_{3}}} \tilde{g}_{a, b} \geq \min _{|z|=r_{3}}\left(\tilde{g}_{a, b}-\chi^{\mathrm{c}} f_{a, b}\right)+\min _{|z|=r_{3}} f_{a, b}-\max _{r_{3} \leq|z| \leq r_{4}} \chi f_{a, b}
$$

These bounds imply that

$$
\begin{aligned}
\max _{X} \tilde{g}_{a, b} & \leq \max _{|z|=r_{3}}\left(\tilde{g}_{a, b}-\chi^{\mathrm{c}} f_{a, b}\right)+2 \sup _{A}\left|f_{a, b}\right|, \\
\min _{X} \tilde{g}_{a, b} & \geq \min _{|z|=r_{3}}\left(\tilde{g}_{a, b}-\chi^{\mathrm{c}} f_{a, b}\right)-2 \sup _{A}\left|f_{a, b}\right|
\end{aligned}
$$

and hence

$$
\max _{X} \tilde{g}_{a, b}-\min _{X} \tilde{g}_{a, b} \leq \max _{|z|=r_{3}}\left(\tilde{g}_{a, b}-\chi^{\mathrm{c}} f_{a, b}\right)-\min _{|z|=r_{3}}\left(\tilde{g}_{a, b}-\chi^{\mathrm{c}} f_{a, b}\right)+4 \sup _{A}\left|f_{a, b}\right| .
$$

By Lemmas A.1 and A.2,

$$
\begin{aligned}
\max _{|z|=r_{3}}\left(\tilde{g}_{a, b}-\chi^{\mathrm{c}} f_{a, b}\right)-\min _{|z|=r_{3}}\left(\tilde{g}_{a, b}-\chi^{\mathrm{c}} f_{a, b}\right) & \leq \frac{2 \sqrt{\pi}}{r_{4}-r_{2}}\left\|d \tilde{g}_{a, b}-d\left(\chi^{\mathrm{c}} f_{a, b}\right)\right\|_{A} \\
& \leq \frac{2 \sqrt{\pi}}{r_{4}-r_{2}}\left(\left\|d \tilde{g}_{a, b}\right\|_{A}+\left\|d\left(\chi^{\mathrm{c}} f_{a, b}\right)\right\|_{A}\right) \\
& \leq \frac{4 \sqrt{\pi}}{r_{4}-r_{2}}\left\|d\left(\chi^{\mathrm{c}} f_{a, b}\right)\right\|_{A} .
\end{aligned}
$$


We have

$$
\begin{aligned}
\left\|d\left(\chi^{\mathrm{c}} f_{a, b}\right)\right\|_{A} & \leq\left\|d\left(\chi^{\mathrm{c}}\right) f_{a, b}\right\|_{A}+\left\|\chi^{\mathrm{c}} d f_{a, b}\right\|_{A} \\
& \leq\left\|\tilde{\chi}^{\prime}(\rho) f_{a, b} d \rho\right\|_{A}+\left\|d f_{a, b}\right\|_{A} \\
& \leq \lambda\|d \rho\|_{A} \sup _{A}\left|f_{a, b}\right|+\left\|d f_{a, b}\right\|_{A} .
\end{aligned}
$$

Now

$$
\|d \rho\|_{A}^{2}=\int_{A} d \rho \wedge * d \rho=\int_{A} \rho d \rho \wedge d \phi=\pi\left(r_{4}^{2}-r_{2}^{2}\right) .
$$

Furthermore, for all $a, b \in U^{r_{1}}$, we have

$\left|f_{a, b}(z)\right|=\frac{1}{2 \pi}|\log | z-z(a)|+\log | \overline{z(a)} z-r_{4}^{2}|-\log | z-z(b)|-\log | \overline{z(b)} z-r_{4}^{2}||$.

For all $a \in U^{r_{1}}$ and all $z \in A$, the triangle inequality gives

$$
r_{2}-r_{1}<|z-z(a)|<r_{4}+r_{1} \quad \text { and } \quad r_{4}\left(r_{4}-r_{1}\right)<\left|\overline{z(a)} z-r_{4}^{2}\right|<r_{4}\left(r_{4}+r_{1}\right) .
$$

From this, we deduce that, for all $a, b \in U^{r_{1}}$,

$$
\sup _{A}\left|f_{a, b}\right| \leq \frac{1}{2 \pi} \log \frac{\left(r_{1}+r_{4}\right)^{2}}{\left(r_{2}-r_{1}\right)\left(r_{4}-r_{1}\right)} .
$$

Finally, we bound the quantity $\left\|d f_{a, b}\right\|_{A}$. Because $f_{a, b}$ is a real function, we have $d f_{a, b}=\partial_{z} f_{a, b} d z+\overline{\partial_{z} f_{a, b}} d \bar{z}$. Therefore,

$$
\begin{aligned}
\left\|d f_{a, b}\right\|_{A}^{2} & =\int_{A} d f_{a, b} \wedge * d f_{a, b}=2 i \int_{A}\left|\partial_{z} f_{a, b}\right|^{2} d z \wedge d \bar{z} \\
& =4 \int_{0}^{2 \pi} \int_{r_{2}}^{1}\left|\partial_{z} f_{a, b}\right|^{2} \rho d \rho d \phi \leq 4 \pi\left(1-r_{2}^{2}\right) \sup _{A}\left|\partial_{z} f_{a, b}\right|^{2} .
\end{aligned}
$$

A straightforward computation gives

$$
\partial_{z} f_{a, b}=\frac{1}{4 \pi}\left(\frac{1}{z-z(a)}+\frac{\overline{z(a)}}{\overline{z(a)} z-r_{4}^{2}}-\frac{1}{z-z(b)}-\frac{\overline{z(b)}}{\overline{z(b)} z-r_{4}^{2}}\right) .
$$

Our previous bounds for $|z-z(a)|$ and $|\overline{z(a)} z-1|$ yield

$$
\sup _{A}\left|\partial_{z} f_{a, b}\right| \leq \frac{1}{2 \pi}\left(\frac{1}{r_{2}-r_{1}}+\frac{r_{1}}{r_{4}\left(r_{4}-r_{1}\right)}\right) .
$$

From this, we obtain

$$
\left\|d f_{a, b}\right\|_{A} \leq \sqrt{\frac{r_{4}^{2}-r_{2}^{2}}{\pi}}\left(\frac{1}{r_{2}-r_{1}}+\frac{r_{1}}{r_{4}\left(r_{4}-r_{1}\right)}\right) .
$$

Combining the bounds for $\sup _{A}\left|f_{a, b}\right|$ and $\left\|d f_{a, b}\right\|_{A}$ yields the lemma. 
From now on, we impose the normalisation condition

$$
\int_{X} \tilde{g}_{a, b} \mu=0
$$

on $\tilde{g}_{a, b}$ for all $a, b \in U^{r_{1}}$; this can be attained by adding a suitable constant to $\tilde{g}_{a, b}$. Then for all $a, b \in U^{r_{1}}$, the function $g_{a, b}$ defined earlier is equal to

$$
g_{a, b}=\tilde{g}_{a, b}+\chi f_{a, b}-\int_{X} \chi f_{a, b} \mu .
$$

Indeed, by the definition of $\tilde{g}_{a, b}$, the right-hand side satisfies (1). Furthermore, for all $a \in U^{r_{1}}$, we define a smooth function $l_{a}$ on $X \backslash\{a\}$ by

$$
l_{a}= \begin{cases}(\chi / 2 \pi) \log |z-z(a)| & \text { on } U, \\ 0 & \text { on } X \backslash \bar{U}\end{cases}
$$

this is bounded from above by $(1 / 2 \pi) \log \left(r_{4}+r_{1}\right)$.

Lemma A.4. For all $a, b \in U^{r_{1}}$, we have

$$
\max _{X}\left|g_{a, b}-l_{a}+l_{b}\right|<c_{4}\left(r_{1}, r_{2}, r_{4}, \lambda, c_{1}\right),
$$

where

$$
c_{4}\left(r_{1}, r_{2}, r_{4}, \lambda, c_{1}\right)=c_{3}\left(r_{1}, r_{2}, r_{4}, \lambda\right)+\frac{1}{2 \pi} \log \frac{r_{4}+r_{1}}{r_{4}-r_{1}}+\left(\frac{8}{3} \log 2-\frac{1}{4}\right) \frac{c_{1}}{r_{4}^{2}} .
$$

Proof. By (3) and the definitions of $f_{a, b}$ and $l_{a}$, we get

$$
g_{a, b}-l_{a}+l_{b}=\tilde{g}_{a, b}-\int_{X} \chi f_{a, b} \mu+\frac{\chi}{2 \pi} \log \left|\frac{\overline{z(a)} z-r_{4}^{2}}{\overline{z(b)} z-r_{4}^{2}}\right|,
$$

where the last term is extended to zero outside $U$. We bound each of the terms on the right-hand side. From $\int_{X} \tilde{g}_{a, b} \mu=0$ and the nonnegativity of $\mu$, it follows that

$$
\max _{X} \tilde{g}_{a, b} \geq 0 \geq \min _{X} \tilde{g}_{a, b}
$$

Together with the bound for $\max _{X} \tilde{g}_{a, b}-\min _{X} \tilde{g}_{a, b}$ from Lemma A.3, this implies

$$
\max _{X}\left|\tilde{g}_{a, b}\right| \leq c_{3}\left(r_{1}, r_{2}, r_{4}, \lambda, c_{1}\right) .
$$

Because the support of $\chi$ is contained in $U^{r_{4}}$, the hypothesis (4) of Definition 3.1.1 together with the definition of $f_{a, b}$ gives

$$
\begin{aligned}
& \int_{X} \chi f_{a, b} \mu \\
& =\int_{U^{r_{4}}} \frac{\chi}{2 \pi}\left(\log \left|\frac{z-z(a)}{r_{4}}\right|+\log \left|\frac{\overline{z(a)} z}{r_{4}^{2}}-1\right|-\log \left|\frac{z-z(b)}{r_{4}}\right|-\log \left|\frac{\overline{z(b)} z}{r_{4}^{2}}-1\right|\right) \mu .
\end{aligned}
$$


Writing $w=z / r_{4}$ and $t=z(a) / r_{4}$, we have

$$
\int_{U^{r_{4}}} \frac{\chi}{2 \pi} \log \left|\frac{z-z(a)}{r_{4}}\right| \mu \leq \frac{c_{1}}{2 \pi r_{4}^{2}} \int_{\substack{|w|<1 \\|w-t|>1}} \log |w-t| i d w \wedge d \bar{w}
$$

We note that $t$ satisfies $|t|<r_{1} / r_{4}$; for simplicity, we relax this to $|t| \leq 1$. Then it is easy to see that the above expression attains its maximum for $|t|=1$; by rotational symmetry, we can take $t=1$. We now have to integrate over the crescent-shaped domain $\{w \in \mathbb{C}:|w|<1$ and $|w-1|>1\}$, which is contained in $\{1+r \exp (i \phi)$ : $1<r<2,2 \pi / 3<\phi<4 \pi / 3\}$. We get

$$
\begin{aligned}
\int_{U^{r_{4}}} \frac{\chi}{2 \pi} \log \left|\frac{z-z(a)}{r_{4}}\right| \mu & <\frac{c_{1}}{\pi} \int_{2 \pi / 3}^{4 \pi / 3} \int_{1}^{2} \log (r) r d r d \phi \\
& =\left(\frac{4}{3} \log 2-\frac{1}{2}\right) c_{1} .
\end{aligned}
$$

In a similar way, we obtain

$$
\begin{aligned}
& \int_{U^{r_{4}}} \frac{\chi}{2 \pi} \log \left|\frac{z-z(a)}{r_{4}}\right| \mu \geq-\frac{c_{1}}{2 r_{4}^{2}}, \\
& \int_{U^{r_{4}}} \frac{\chi}{2 \pi} \log \left|\frac{\overline{z(a)} z}{r_{4}^{2}}-1\right| \mu<\left(\frac{4}{3} \log 2-\frac{1}{2}\right) \frac{c_{1}}{r_{4}^{2}}, \\
& \int_{U^{r_{4}}} \frac{\chi}{2 \pi} \log \left|\frac{\overline{z(a)} z}{r_{4}^{2}}-1\right| \mu \geq-\frac{c_{1}}{4 r_{4}^{2}} .
\end{aligned}
$$

The same bounds hold for $b$. Combining everything, we get

$$
\left|\int_{X} \chi f_{a, b} \mu\right| \leq\left(\frac{8}{3} \log 2-\frac{1}{4}\right) \frac{c_{1}}{r_{4}^{2}} \text {. }
$$

Finally, we have

$$
\begin{aligned}
\max _{X} \frac{\chi}{2 \pi} \log \left|\frac{\overline{z(a)} z-r_{4}^{2}}{\overline{z(b)} z-r_{4}^{2}}\right| & \leq \frac{1}{2 \pi} \sup _{U^{r_{4}}} \log \left|\frac{r_{4}-\overline{z(a)} z / r_{4}}{r_{4}-\overline{z(b)} z / r_{4}}\right| \\
& \leq \frac{1}{2 \pi} \log \frac{r_{4}+r_{1}}{r_{4}-r_{1}},
\end{aligned}
$$

which finishes the proof.

We will now apply Lemma A.4, which holds for any chart $(U, z)$ satisfying the hypotheses (1) and (4) of Definition 3.1.1, to our atlas $\left\{\left(U_{j}, z_{j}\right): 1 \leq j \leq n\right\}$. Besides including the index $j$ in the notation for the coordinates, we denote by $l_{a}^{(j)}$ and $\chi^{(j)}$ the functions $l_{a}$ and $\chi$ defined for the coordinate $\left(U_{j}, z_{j}\right)$. We obtain the following generalisation of Lemma A.4 to the situation where $a$ and $b$ are arbitrary points of $X$ : 
Lemma A.5. For all $a, b \in X$ and all $j$ and $k$ such that $a \in U_{j}^{r_{1}}$ and $b \in U_{k}^{r_{1}}$,

$$
\sup _{X}\left|g_{a, b}-l_{a}^{(j)}+l_{b}^{(k)}\right| \leq c_{5}\left(r_{1}, r_{2}, r_{4}, \lambda, n, c_{1}, M\right),
$$

where

$$
c_{5}\left(r_{1}, r_{2}, r_{4}, \lambda, c_{1}, n, M\right)=n c_{4}\left(r_{1}, r_{2}, r_{4}, \lambda, c_{1}\right)+\frac{n-1}{2 \pi} \log \left(M \frac{r_{4}+r_{1}}{r_{2}-r_{1}}\right) .
$$

Proof. We first show that for any two coordinate indices $j$ and $k$ and for all $a \in U_{k}^{r_{1}} \cap U_{j}^{r_{1}}$,

$$
\sup _{X}\left|l_{a}^{(k)}-l_{a}^{(j)}\right| \leq \frac{1}{2 \pi} \log \left(M \frac{r_{4}+r_{1}}{r_{2}-r_{1}}\right) .
$$

To prove this, let $y \in X$. We distinguish three cases to prove that $l_{a}^{(k)}(y)-l_{a}^{(j)}(y)$ is bounded from above by the right-hand side of (4); the inequality then follows by interchanging $j$ and $k$.

Case 1. Suppose $y \in U_{j}$ with $\left|z_{j}(y)-z_{j}(a)\right|<\left(r_{2}-r_{1}\right) / M$. In this case, we have

$$
\left|z_{j}(y)\right|<\left|z_{j}(a)\right|+\frac{r_{2}-r_{1}}{M}<r_{2} ;
$$

hence, $a, y \in U_{j}^{r_{2}}$. Let $[a, y]^{j}$ denote the line segment between $a$ and $y$ in the $z_{j}$-coordinate, i.e., the curve in $U_{j}^{r_{2}}$ whose $z_{j}$-coordinate is parametrised by

$$
\hat{z}_{j}(t)=(1-t) z_{j}(a)+t z_{j}(y) \text { for } 0 \leq t \leq 1 .
$$

We claim that this line segment also lies inside $U_{k}^{r_{2}}$. Suppose this is not the case; then, because the "starting point" $z_{j}^{-1}\left(\hat{z}_{j}(0)\right)=a$ does lie in $U_{k}^{r_{2}}$, there exists a smallest $t \in(0,1)$ for which the point

$$
y^{\prime}=z_{j}^{-1}\left(\hat{z}_{j}(t)\right) \in U_{j}^{r_{2}}
$$

lies on the boundary of $U_{k}^{r_{2}}$. It follows from hypothesis (3) of Definition 3.1.1 that

$$
\left|z_{k}\left(y^{\prime}\right)-z_{k}(a)\right| \leq M\left|z_{j}\left(y^{\prime}\right)-z_{j}(a)\right| \text {. }
$$

On the other hand,

$$
\begin{aligned}
\left|z_{j}\left(y^{\prime}\right)-z_{j}(a)\right| & =t\left|z_{j}(y)-z_{j}(a)\right| \\
& <\left(r_{2}-r_{1}\right) / M
\end{aligned}
$$

by assumption, and

$$
\left|z_{k}\left(y^{\prime}\right)-z_{k}(a)\right|>r_{2}-r_{1}
$$

by the triangle inequality. This implies

$$
\left|z_{k}\left(y^{\prime}\right)-z_{k}(a)\right|>M\left|z_{j}\left(y^{\prime}\right)-z_{j}(a)\right|,
$$


a contradiction. Therefore, the line segment $[a, y]^{j}$ lies inside $U_{j}^{r_{2}} \cap U_{k}^{r_{2}}$. By hypothesis (3) of Definition 3.1.1, we have

$$
\left|z_{k}(y)-z_{k}(a)\right| \leq M\left|z_{j}(y)-z_{j}(a)\right| .
$$

Because $\chi^{(j)}(y)=\chi^{(k)}(y)=1$, we find

$$
l_{a}^{(k)}(y)-l_{a}^{(j)}(y)=\frac{1}{2 \pi} \log \left|\frac{z_{k}(y)-z_{k}(a)}{z_{j}(y)-z_{j}(a)}\right| \leq \frac{1}{2 \pi} \log M,
$$

which is bounded by the right-hand side of (4).

Case 2. Suppose $y \notin U_{j}$. Then $l_{a}^{(j)}(y)=0$, and thus,

$$
l_{a}^{(k)}(y)-l_{a}^{(j)}(y)=l_{a}^{(k)}(y) \leq \frac{\log \left(r_{4}+r_{1}\right)}{2 \pi} .
$$

Case 3. Suppose $y \in U_{j}$ and $\left|z_{j}(y)-z_{j}(a)\right| \geq\left(r_{2}-r_{1}\right) / M$. Then

$$
l_{a}^{(k)}(y)-l_{a}^{(j)}(y) \leq \frac{\log \left(r_{4}+r_{1}\right)}{2 \pi}-\frac{\chi^{(j)}(y)}{2 \pi} \log \frac{r_{2}-r_{1}}{M},
$$

which is also bounded by the right-hand side in (4).

By hypothesis (2) of Definition 3.1.1, the open sets $U_{j}^{r_{1}}$ cover $X$. Furthermore, $X$ is connected. For arbitrary $a, b \in X$ and indices $j$ and $k$ such that $a \in U_{j}^{r_{1}}$ and $b \in U_{k}^{r_{1}}$, we can therefore choose a finite sequence of indices $j=j_{1}, j_{2}, \ldots, j_{m}=k$ with $m \leq n$ and points $a=a_{0}, a_{1}, \ldots, a_{m}=b$ such that $a_{i} \in U_{j_{i}}^{r_{1}} \cap U_{j_{i+1}}^{r_{1}}$ for $1 \leq i \leq m-1$. Using $g_{a, b}=\sum_{i=1}^{m} g_{a_{i-1}, a_{i}}$, we get

$$
\begin{aligned}
\sup _{X}\left|g_{a, b}-l_{a}^{(j)}+l_{b}^{(k)}\right| & =\sup _{X}\left|\sum_{i=1}^{m}\left(g_{a_{i-1}, a_{i}}-l_{a_{i-1}}^{\left(j_{j}\right)}+l_{a_{i}}^{\left(j_{j}\right)}\right)+\sum_{i=1}^{m-1}\left(l_{a_{i}}^{\left(j_{i+1}\right)}-l_{a_{i}}^{\left(j_{i}\right)}\right)\right| \\
& \leq \sum_{i=1}^{m} \sup _{X}\left|g_{a_{i-1}, a_{i}}-l_{a_{i-1}}^{\left(j_{i}\right)}+l_{a_{i}}^{\left(j_{i}\right)}\right|+\sum_{i=1}^{m-1} \sup _{X}\left|l_{a_{i}}^{\left(j_{i+1}\right)}-l_{a_{i}}^{\left(j_{i}\right)}\right| .
\end{aligned}
$$

The lemma now follows from Lemma A.4 and the inequality (4).

Proof of Theorem 3.1.2. We choose a continuous partition of unity $\left\{\phi^{j}\right\}_{j=1}^{n}$ subordinate to the covering $\left\{U_{j}^{r_{1}}\right\}_{j=1}^{n}$. Let $a \in X$, and let $j$ be an index such that $a \in U_{j}^{r_{1}}$. By the definition of $g_{a, \mu}$, we have

$$
\begin{aligned}
g_{a, \mu}(x)-l_{a}^{(j)}(x) & =\int_{b \in X} g_{a, b}(x) \mu(b)-l_{a}^{(j)}(x) \\
& =\sum_{k=1}^{n} \int_{b \in U_{k}^{r_{1}}} \phi^{k}(b)\left(g_{a, b}(x)-l_{a}^{(j)}(x)\right) \mu(b)
\end{aligned}
$$


$=\sum_{k=1}^{n} \int_{b \in U_{k}^{r_{1}}} \phi^{k}(b)\left(g_{a, b}(x)-l_{a}^{(j)}(x)+l_{b}^{(k)}(x)\right) \mu(b)-\sum_{k=1}^{n} \int_{b \in U_{k}^{r_{1}}} \phi^{k}(b) l_{b}^{(k)}(x) \mu(b)$.

In a similar way to in the proof of Lemma A.4, one can check that, for every index $k$ and all $x \in X$, we have

$$
-\frac{c_{1}}{2} \leq \int_{b \in U_{k}^{r_{1}}} \phi^{k}(b) l_{b}^{(k)}(x) \mu(b) \leq\left(\frac{4}{3} \log 2-\frac{1}{2}\right) c_{1}
$$

so that

$$
\sup _{x \in X}\left|\int_{b \in U_{k}^{r_{1}}} \phi^{k}(b) l_{b}^{(k)}(x) \mu(b)\right| \leq \frac{c_{1}}{2} .
$$

Together with Lemma A.5, this gives the inequality

$$
\begin{aligned}
\sup _{X}\left|g_{a, \mu}-l_{a}^{(j)}\right| & \leq c_{5}\left(r_{1}, r_{2}, r_{4}, \lambda, c_{1}, n, M\right) \sum_{j=1}^{n} \int_{b \in U_{j}^{r_{1}}} \phi^{j}(b) \mu(b)+\sum_{j=1}^{n} \frac{c_{1}}{2} \\
& =c_{5}\left(r_{1}, r_{2}, r_{4}, \lambda, c_{1}, n, M\right)+\frac{n c_{1}}{2} .
\end{aligned}
$$

We also have

$$
\sup _{X} g_{a, \mu} \leq \sup _{X}\left(g_{a, \mu}-l_{a}^{(j)}\right)+\sup _{X} l_{a}^{(j)} \leq \sup _{X}\left(g_{a, \mu}-l_{a}^{(j)}\right)+\frac{\log \left(r_{4}+r_{1}\right)}{2 \pi} .
$$

By varying the choice of $r_{4}$ and $\tilde{\chi}$, we can let $r_{4}$ tend to 1 and $\lambda$ to $\frac{1}{1-r_{2}}$. This leads to

$$
\begin{array}{r}
c_{3}\left(r_{1}, r_{2}, 1, \frac{1}{1-r_{2}}\right)=4 \sqrt{\frac{1+r_{2}}{1-r_{2}}}\left(\frac{1}{2\left(1-r_{2}\right)} \log \frac{\left(r_{1}+1\right)^{2}}{\left(r_{2}-r_{1}\right)\left(1-r_{1}\right)}+\frac{1}{r_{2}-r_{1}}+\frac{r_{1}}{1-r_{1}}\right) \\
+\frac{2}{\pi} \log \frac{\left(r_{1}+1\right)^{2}}{\left(r_{2}-r_{1}\right)\left(1-r_{1}\right)},
\end{array}
$$

which implies successively

$$
\begin{aligned}
c_{4}\left(r_{1}, r_{2}, 1, \frac{1}{1-r_{2}}, c_{1}\right) & =c_{3}\left(r_{1}, r_{2}, 1, \frac{1}{1-r_{2}}\right)+\frac{1}{2 \pi} \log \frac{1+r_{1}}{1-r_{1}}+\left(\frac{8}{3} \log 2-\frac{1}{4}\right) c_{1}, \\
c_{5} & =n c_{4}\left(r_{1}, r_{2}, r_{4}, \frac{1}{1-r_{2}}, c_{1}\right)+\frac{n-1}{2 \pi} \log \left(M \frac{1+r_{1}}{r_{2}-r_{1}}\right) .
\end{aligned}
$$

We take $r_{2}=0.39+0.61 r_{1}$. Then, for $r_{1}>\frac{1}{2}$, one can check numerically that

$$
c_{5} \leq 52.4 \frac{n}{\left(1-r_{1}\right)^{3 / 2}} \log \frac{1}{1-r_{1}}+1.60 n c_{1}+\frac{n-1}{2 \pi} \log M .
$$

From this, the theorem follows. 


\section{Acknowledgements}

I thank Peter Bruin, Bas Edixhoven and Robin de Jong. They introduced me to Arakelov theory and Merkl's theorem, and I am grateful to them for many inspiring discussions and their help in writing this article. I also thank Rafael von Känel and Jan Steffen Müller for motivating discussions about this article. I thank JeanBenoît Bost and Gerard Freixas for discussions on Arakelov geometry, Yuri Bilu for inspiring discussions on Riemann's existence theorem, Jürg Kramer for discussions on Faltings' delta invariant, Hendrik Lenstra and Bart de Smit for their help in proving Proposition 4.1.1, Qing Liu for answering my questions on models of finite morphisms of curves and Karl Schwede for helpful discussions about the geometry of surfaces.

\section{References}

[Abbes and Ullmo 1997] A. Abbes and E. Ullmo, "Auto-intersection du dualisant relatif des courbes modulaires $X_{0}(N)$ ”, J. Reine Angew. Math. 484 (1997), 1-70. MR 99e:11077 Zbl 0934.14016

[Arakelov 1974] S. J. Arakelov, "Intersection theory for divisors on an arithmetic surface", Izv. Akad. Nauk SSSR Ser. Mat. 38 (1974), 1179-1192. In Russian; translated in Math. USSR, Izv. 8:6 (1974), 1167-1180. MR 57 \#12505 Zbl 0355.14002

[Belyi 1979] G. V. Belyi, "On Galois extensions of a maximal cyclotomic field”, Izv. Akad. Nauk SSSR Ser. Mat. 43:2 (1979), 267-276. In Russian; translated in Math. USSR, Izv. 14:2 (1980), 247-256. MR 80f:12008 Zbl 0429.12004

[Bilu and Strambi 2010] Y. F. Bilu and M. Strambi, "Quantitative Riemann existence theorem over a number field”, Acta Arith. 145:4 (2010), 319-339. MR 2011m:14045 Zbl 1222.11082

[Bruin 2010] P. Bruin, Modular curves, Arakelov theory, algorithmic applications, Ph.D. thesis, Universiteit Leiden, 2010, http://www.math.leidenuniv.nl/scripties/proefschrift-peterbruin.pdf.

[Bruin 2011] P. Bruin, "Computing coefficients of modular forms", pp. 19-36 in Actes de la Conférence "Théorie des Nombres et Applications", Presses Univ. Franche-Comté, Besançon, 2011. MR 2894266 Zbl 1267.11036

[Bruin 2013] P. Bruin, "Explicit bounds on automorphic and canonical Green functions of Fuchsian groups", preprint, 2013, http://homepages.warwick.ac.uk/staff/P.Bruin/green.pdf. To appear in Mathematika.

[Clark and Voight 2011] P. L. Clark and J. Voight, "Algebraic curves uniformized by congruence subgroups of triangle groups", preprint, 2011, http://math.uga.edu/ pete/triangle-072011.pdf.

[Curilla and Kühn 2009] C. Curilla and U. Kühn, "On the arithmetic self-intersection numbers of the dualizing sheaf for Fermat curves of prime exponent", preprint, 2009. arXiv 0906.3891v1

[David 1991] S. David, "Fonctions thêta et points de torsion des variétés abéliennes", Compositio Math. 78:2 (1991), 121-160. MR 92d:11061 Zbl 0741.14025

[Edixhoven and de Jong 2011a] B. Edixhoven and R. de Jong, "Bounds for Arakelov invariants of modular curves", pp. 217-256 in Computational aspects of modular forms and Galois representations, edited by B. Edixhoven and J.-M. Couveignes, Ann. of Math. Stud. 176, Princeton Univ. Press, 2011. MR 2857095 Zbl 1216.11004

[Edixhoven and de Jong 2011b] B. Edixhoven and R. de Jong, "Short introduction to heights and Arakelov theory", pp. 79-94 in Computational aspects of modular forms and Galois representations, 
edited by B. Edixhoven and J.-M. Couveignes, Ann. of Math. Stud. 176, Princeton Univ. Press, 2011. MR 2867563 Zbl 1216.11004

[Edixhoven et al. 2010] B. Edixhoven, R. de Jong, and J. Schepers, "Covers of surfaces with fixed branch locus", Internat. J. Math. 21:7 (2010), 859-874. MR 2011i:14032 Zbl 1203.14021

[Faltings 1983] G. Faltings, "Endlichkeitssätze für abelsche Varietäten über Zahlkörpern”, Invent. Math. 73:3 (1983), 349-366. MR 85g:11026a Zbl 0588.14026

[Faltings 1984] G. Faltings, "Calculus on arithmetic surfaces”, Ann. of Math. (2) 119:2 (1984), 387-424. MR 86e:14009 Zbl 0559.14005

[Gaudron and Rémond 2011] E. Gaudron and G. Rémond, "Théorème des periodes et degrés minimaux d'isogénies", preprint, 2011. To appear in Comment. Math. Helv. arXiv 1105.1230v1

[Graftieaux 2001] P. Graftieaux, "Formal groups and the isogeny theorem", Duke Math. J. 106:1 (2001), 81-121. MR 2002f:14055 Zbl 1064.14045

[Igusa 1972] J.-i. Igusa, Theta functions, Die Grundlehren der mathematischen Wissenschaften 194, Springer, New York, 1972. MR 48 \#3972 Zbl 0251.14016

[Javanpeykar and von Känel 2013] A. Javanpeykar and R. von Känel, “Szpiro's small points conjecture for cyclic covers", preprint, 2013. arXiv 1311.0043v1

[de Jong 2005a] R. de Jong, "Arakelov invariants of Riemann surfaces", Doc. Math. 10 (2005), 311-329. MR 2006j:14030 Zbl 1087.14023

[de Jong 2005b] R. de Jong, "On the Arakelov theory of elliptic curves", Enseign. Math. (2) 51:3-4 (2005), 179-201. MR 2007b:14047 Zbl 1115.14014

[Jorgenson and Kramer 2004] J. Jorgenson and J. Kramer, "Bounding the sup-norm of automorphic forms”, Geom. Funct. Anal. 14:6 (2004), 1267-1277. MR 2005m:11071 Zbl 1078.11027

[Jorgenson and Kramer 2006] J. Jorgenson and J. Kramer, "Bounds on canonical Green's functions", Compos. Math. 142:3 (2006), 679-700. MR 2007h:14030 Zbl 1105.14028

[Jorgenson and Kramer 2009] J. Jorgenson and J. Kramer, "Bounds on Faltings's delta function through covers", Ann. of Math. (2) 170:1 (2009), 1-43. MR 2010g:14031 Zbl 1169.14020

[Khadjavi 2002] L. S. Khadjavi, "An effective version of Belyi's theorem”, J. Number Theor. 96:1 (2002), 22-47. MR 2003h:11072 Zbl 1078.11046

[Kühn 2013] U. Kühn, "On the arithmetic self-intersection number of the dualizing sheaf on arithmetic surfaces”, preprint, 2013. arXiv 0906.2056v2

[Lițcanu 2004] R. Lițcanu, "Propriétés du degré des morphismes de Belyi", Monatsh. Math. 142:4 (2004), 327-340. MR 2005i:11082 Zbl 1078.14034

[Liu 2006a] Q. Liu, Algebraic geometry and arithmetic curves, 2nd ed., Oxford Graduate Texts in Mathematics 6, Oxford University Press, Oxford, 2006. MR 2003g:14001 Zbl 1103.14001

[Liu 2006b] Q. Liu, "Stable reduction of finite covers of curves", Compos. Math. 142:1 (2006), 101-118. MR 2007k:14057 Zbl 1108.14020

[Long 2008] L. Long, "Finite index subgroups of the modular group and their modular forms", pp. 83-102 in Modular forms and string duality, edited by N. Yui et al., Fields Inst. Commun. 54, Amer. Math. Soc., Providence, RI, 2008. MR 2009k:11069 Zbl 1163.11032

[Mayer 2012] H. Mayer, Self-intersection of the dualizing sheaf of modular curves $X_{1}(n)$, Ph.D. thesis, Humboldt-Universität zu Berlin, 2012. arXiv 1212.1294v1

[Merkl 2011] F. Merkl, "An upper bound for Green functions on Riemann surfaces", pp. 203-215 in Computational aspects of modular forms and Galois representations, edited by B. Edixhoven and J.M. Couveignes, Ann. of Math. Stud. 176, Princeton Univ. Press, 2011. MR 2857094 Zbl 1216.11004

[Michel and Ullmo 1998] P. Michel and E. Ullmo, "Points de petite hauteur sur les courbes modulaires $X_{0}(N)$ ", Invent. Math. 131:3 (1998), 645-674. MR 99c:11074 Zbl 0991.11037 
[Moret-Bailly 1989] L. Moret-Bailly, "La formule de Noether pour les surfaces arithmétiques", Invent. Math. 98:3 (1989), 491-498. MR 91h:14023 Zbl 0727.14014

[Moret-Bailly 1990] L. Moret-Bailly, "Hauteurs et classes de Chern sur les surfaces arithmétiques", pp. 37-58 in Séminaire sur les Pinceaux de Courbes Elliptiques (Paris, 1988), Astérisque 183, Soc. Math. de France, Paris, 1990. MR 92g:14018a Zbl 0727.14015

[Pazuki 2012] F. Pazuki, “Theta height and Faltings height”, Bull. Soc. Math. France 140:1 (2012), 19-49. MR 2903770 Zbl 1245.14029

[Rémond 1999] G. Rémond, "Hauteurs thêta et construction de Kodaira", J. Number Theory 78:2 (1999), 287-311. MR 2000g:11059 Zbl 0947.14016

[Serre 1979] J.-P. Serre, Local fields, Graduate Texts in Mathematics 67, Springer, New York, 1979. MR 82e:12016 Zbl 0423.12016

[Szpiro 1985a] L. Szpiro, "La conjecture de Mordell (d'après G. Faltings)”, pp. 83-103 in Séminaire Bourbaki (Paris, 1983-1984), Astérisque 121-122, Soc. Math. de France, Paris, 1985. MR 87c:11033 Zbl 0591.14027

[Szpiro 1985b] L. Szpiro (editor), Séminaire sur les pinceaux arithmétiques: la conjecture de Mordell (Paris, 1983-1984), Astérisque 127, Soc. Math. de France, Paris, 1985. MR 87h:14017 Zbl 0588.14028

[Szpiro 1985c] L. Szpiro, "Un peu d'effectivité”, pp. 275-287 in Séminaire sur les pinceaux arithmétiques: La conjecture de Mordell (Paris, 1983-1984), Astérisque 127, Soc. Math. de France, Paris, 1985. MR 801928 Zbl 1182.11016

[Szpiro 1986] L. Szpiro, “Small points and torsion points”, pp. 251-260 in The Lefschetz centennial conference, I (Mexico City, 1984), edited by D. Sundararaman, Contemp. Math. 58, Amer. Math. Soc., Providence, RI, 1986. MR 87k:14029

[Szpiro 1990a] L. Szpiro, "Discriminant et conducteur des courbes elliptiques", pp. 7-18 in Séminaire sur les Pinceaux de Courbes Elliptiques (Paris, 1988), Astérisque 183, Soc. Math. de France, Paris, 1990. MR 91g:11059 Zbl 0742.14026

[Szpiro 1990b] L. Szpiro, "Sur les propriétés numériques du dualisant relatif d'une surface arithmétique”, pp. 229-246 in The Grothendieck Festschrift, vol. III, edited by P. Cartier et al., Progr. Math. 88, Birkhäuser, Boston, MA, 1990. MR 92c:14017 Zbl 0759.14018

[Ullmo 2000] E. Ullmo, "Hauteur de Faltings de quotients de $J_{0}(N)$, discriminants d'algèbres de Hecke et congruences entre formes modulaires", Am. J. Math. 122 (2000), 83-115. MR 2000k:11080 Zbl 0991.11033

[Wolfart 1997] J. Wolfart, “The 'obvious' part of Belyi's theorem and Riemann surfaces with many automorphisms", pp. 97-112 in Geometric Galois actions, 1, edited by L. Schneps and P. Lochak, London Math. Soc. Lecture Note Ser. 242, Cambridge Univ. Press, 1997. MR 99a:14036 Zbl 0915.14021

[Zograf 1991] P. Zograf, “A spectral proof of Rademacher's conjecture for congruence subgroups of the modular group", J. Reine Angew. Math. 414 (1991), 113-116. MR 92d:11041 Zbl 0709.11031

Communicated by Joseph Silverman

Received 2012-06-22 Revised 2013-02-27 Accepted 2013-04-17

ajavanp@math.leidenuniv.nl Mathematisch Instituut, Universiteit Leiden, 2717 GA Leiden, Netherlands

peter.bruin@math.uzh.ch Institut für Mathematik, Universität Zürich, Winterthurerstrasse 190, CH-8057 Zürich, Switzerland 


\section{Algebra \& Number Theory}

msp.org/ant

\section{EDITORS}

MANAGING EDITOR

Bjorn Poonen

Massachusetts Institute of Technology

Cambridge, USA

\author{
EDITORIAL BOARD CHAIR \\ David Eisenbud \\ University of California \\ Berkeley, USA
}

\section{BOARD OF EDITORS}

Georgia Benkart

Dave Benson

Richard E. Borcherds

John H. Coates

J-L. Colliot-Thélène

Brian D. Conrad

Hélène Esnault

Hubert Flenner

Edward Frenkel

Andrew Granville

Joseph Gubeladze

Roger Heath-Brown

Ehud Hrushovski

Craig Huneke

Mikhail Kapranov

Yujiro Kawamata

János Kollár

Yuri Manin

Barry Mazur

Philippe Michel
University of Wisconsin, Madison, USA

University of Aberdeen, Scotland

University of California, Berkeley, USA

University of Cambridge, UK

CNRS, Université Paris-Sud, France

University of Michigan, USA

Freie Universität Berlin, Germany

Ruhr-Universität, Germany

University of California, Berkeley, USA

Université de Montréal, Canada

San Francisco State University, USA

Oxford University, UK

Hebrew University, Israel

University of Virginia, USA

Yale University, USA

University of Tokyo, Japan

Princeton University, USA

Northwestern University, USA

Harvard University, USA

École Polytechnique Fédérale de Lausanne
Susan Montgomery

Shigefumi Mori

Raman Parimala

Jonathan Pila

Victor Reiner

Karl Rubin

Peter Sarnak

Joseph H. Silverman

Michael Singer

Vasudevan Srinivas

J. Toby Stafford

Bernd Sturmfels

Richard Taylor

Ravi Vakil

Michel van den Bergh

Marie-France Vignéras

Kei-Ichi Watanabe

Efim Zelmanov

Shou-Wu Zhang
University of Southern California, USA

RIMS, Kyoto University, Japan

Emory University, USA

University of Oxford, UK

University of Minnesota, USA

University of California, Irvine, USA

Princeton University, USA

Brown University, USA

North Carolina State University, USA

Tata Inst. of Fund. Research, India

University of Michigan, USA

University of California, Berkeley, USA

Harvard University, USA

Stanford University, USA

Hasselt University, Belgium

Université Paris VII, France

Nihon University, Japan

University of California, San Diego, USA

Princeton University, USA

PRODUCTION

production@msp.org

Silvio Levy, Scientific Editor

See inside back cover or msp.org/ant for submission instructions.

The subscription price for 2014 is US $\$ 225 /$ year for the electronic version, and $\$ 400 /$ year $(+\$ 55$, if shipping outside the US) for print and electronic. Subscriptions, requests for back issues and changes of subscribers address should be sent to MSP.

Algebra \& Number Theory (ISSN 1944-7833 electronic, 1937-0652 printed) at Mathematical Sciences Publishers, 798 Evans Hall \#3840, c/o University of California, Berkeley, CA 94720-3840 is published continuously online. Periodical rate postage paid at Berkeley, CA 94704, and additional mailing offices.

ANT peer review and production are managed by EditFLOW ${ }^{\circledR}$ from Mathematical Sciences Publishers.

\section{PUBLISHED BY}

- mathematical sciences publishers

nonprofit scientific publishing

http://msp.org/

(C) 2014 Mathematical Sciences Publishers 


\section{Algebra \& Number Theory}

\section{Volume $8 \quad$ No. $1 \quad 2014$}

On the Picard number of K3 surfaces over number fields

FRANÇOIS CHARLES

Adèle residue symbol and Tate's central extension for multiloop Lie algebras

OLIVER BRAUNLING

On the number of cubic orders of bounded discriminant having automorphism group $C_{3}$, and related problems

MANJUl Bhargava and ARIEl ShNidman

Polynomial bounds for Arakelov invariants of Belyi curves

ARIYAN JAVANPEYKAR

The radius of a subcategory of modules

HAILONG DAO and RYO TAKAHASHI

A generalized Bogomolov-Gieseker inequality for the three-dimensional projective space

EMANUELE MACRÌ

$(\varphi, \Gamma)$-modules over noncommutative overconvergent and Robba rings

GERGELY ZÁBRÁDI

The Tannakian formalism and the Langlands conjectures

DAVID KAZHDAN, MICHAEL LARSEN and YAKOV VARSHAVSKY 